WALDEN

UNIVERSITY

$A$ higher degree. $A$ higher purpose.

Walden University

ScholarWorks

Harold L. Hodgkinson Award for Outstanding

Dissertation

University Awards

2005

\title{
Information-processing skills related to working memory in individuals with Asperger's disorder
}

Nancy Lynn Musarra

Follow this and additional works at: http://scholarworks.waldenu.edu/hodgkinson

This Dissertation is brought to you for free and open access by the University Awards at ScholarWorks. It has been accepted for inclusion in Harold L.

Hodgkinson Award for Outstanding Dissertation by an authorized administrator of ScholarWorks. For more information, please contact

ScholarWorks@waldenu.edu. 


\title{
Walden University
}

\author{
SCHOOL OF PSYCHOLOGY
}

This is to certify that the doctoral dissertation by

\author{
Nancy Lynn Musarra
}

has been found to be complete and satisfactory in all respects, and that any and all revisions required by the review committee have been made.

Review Committee

Dr. Rodney Ford, Committee Chairperson, Psychology Faculty Dr. Joseph Rocchio, Committee Member, Psychology Faculty Dr. S. David Kriska, Committee Member, Psychology Faculty Dr. Hilda Glazer, School Representative, Psychology Faculty

President and Provost

Paula E. Peinovich, Ph.D.

Walden University 2005 


\author{
ABSTRACT \\ Asperger's Disorder \\ by \\ Nancy Lynn Musarra \\ M.S., University of Dayton, 1991 \\ M.S., Hahnemann University, 1986 \\ B.S., Bowling Green State University, 1984 \\ Dissertation Submitted in Partial Fulfillment \\ of the Requirements for the Degree \\ of Doctor of Philosophy \\ Psychology
}

Information-Processing Skills Related to Working Memory in Individuals with 


\title{
Walden University
}

November 2005

\begin{abstract}
The present study examined information-processing (IP) deficits specific to the ability of individuals with Asperger's disorder (AD) to interpret and respond to nonverbal and verbal information inherent in social relationships as it relates to working memory capacity. Sixty boys between the ages of 11.0 and 15.7 years (30 diagnosed with AD [Group A], and 30 typically developing, same-age peers [Group T]) were assessed using the Working Memory Test Battery for Children (WMTB-C). The present study combined theories from cognitive, neurological, and clinical psychology, isolated specific working memory components, and identified a connection between working memory capacity and the social skill deficiencies of individuals with AD. Three working memory scores (i.e., verbal [PL], visual-spatial [VSSP], and complex [CE]) were compared between the two groups using ANOVA. All working memory differences examined between the two groups in the present study were statistically different. The effect sizes of differences between Groups A and T for PL, VSSP, and CE were .397, .279, and .627, respectively. The results of the present study support the hypothesis that working memory is a specific IP deficit of individuals with AD. Findings suggest that by targeting remedial efforts to enhance working memory capacity, individuals with AD can more effectively engage in complex IP tasks, participate in reciprocal social interactions, and thereby create social change. Future research needs to expand upon the connection between working memory capacity and the social deficiencies of individuals with Asperger's disorder.
\end{abstract}


Information-Processing Skills Related to Working Memory in Individuals with Asperger's Disorder

by

\author{
Nancy Lynn Musarra
}

M.S., University of Dayton, 1991

M.S., Hahnemann University, 1986

B.S., Bowling Green State University, 1984

\author{
Dissertation Submitted in Partial Fulfillment \\ of the Requirements for the Degree \\ of Doctor of Philosophy \\ Psychology
}

Walden University

November 2005 
UMI Number: 3188007

Copyright 2006 by

Musarra, Nancy Lynn

All rights reserved.

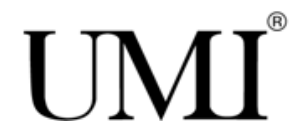

UMI Microform 3188007

Copyright 2006 by ProQuest Information and Learning Company.

All rights reserved. This microform edition is protected against unauthorized copying under Title 17, United States Code.

ProQuest Information and Learning Company 300 North Zeeb Road

P.O. Box 1346

Ann Arbor, MI 48106-1346 


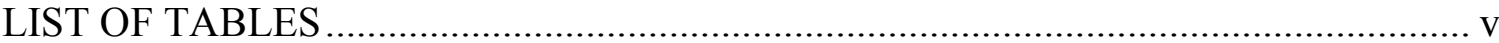

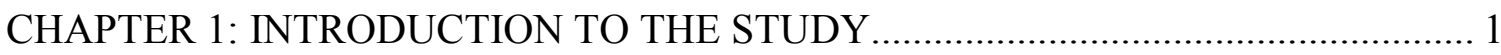

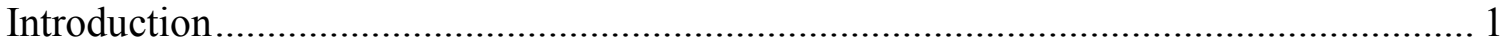

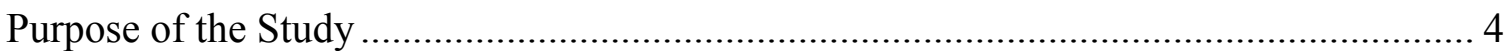

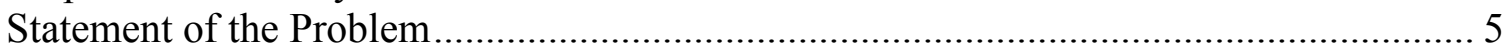

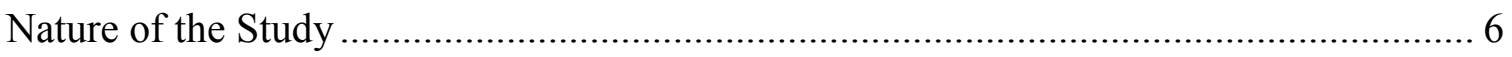

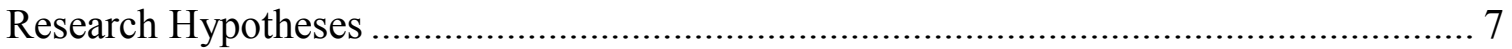

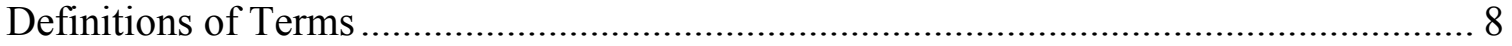

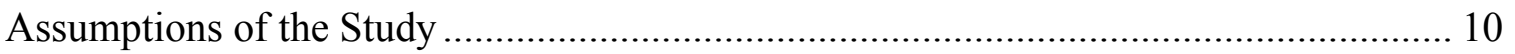

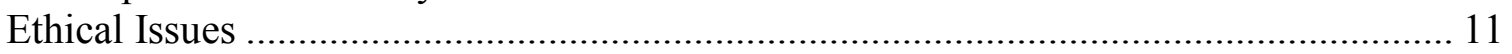

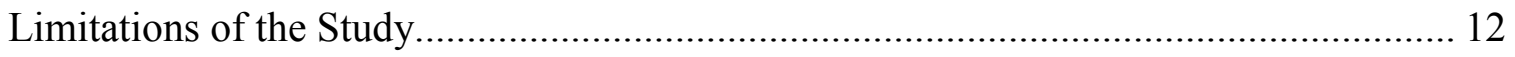

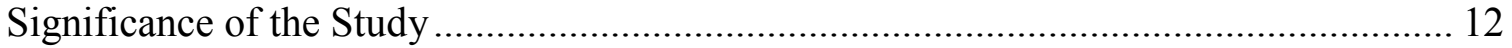

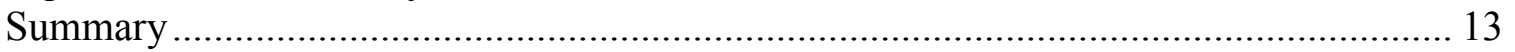

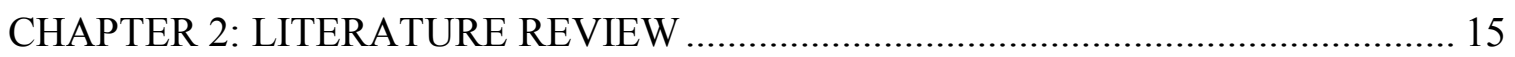

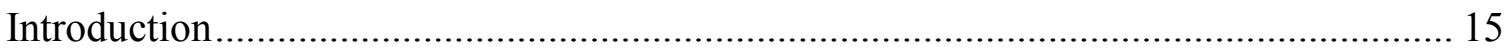

History and Development of Working Memory Theory ………………....................... 16

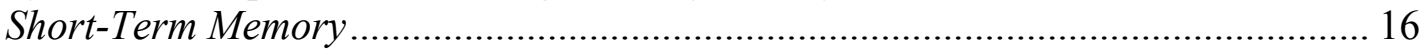

Working Memory Model ................................................................................ 18

Complex Span Tasks of Short-Term Memory ………….............................................. 20

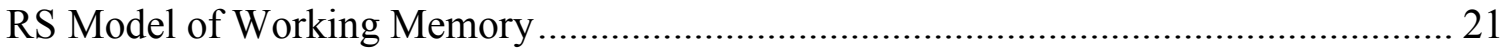

MRS Model of Working Memory ……………………................................................ 23

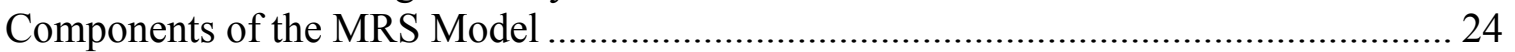

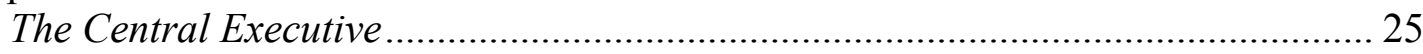

The Phonological Loop ................................................................................... 26

The Visual-Spatial Sketch Pad.......................................................................... 30

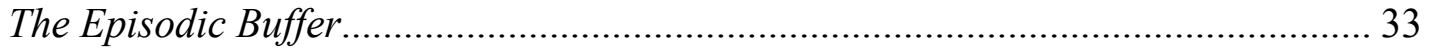

The Development of Working Memory ……………………...................................... 33

The Assessment of Working Memory in Children .......................................................... 38

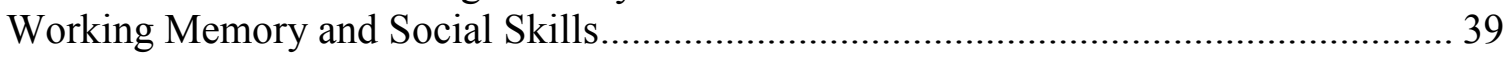

Asperger's Disorder and Social Deficit ..................................................................... 40

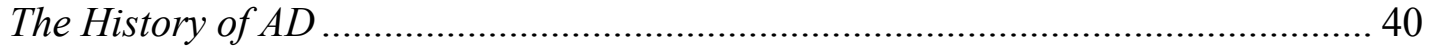

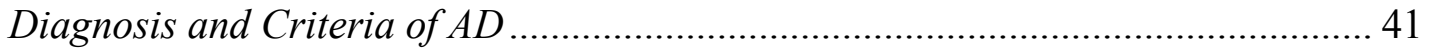

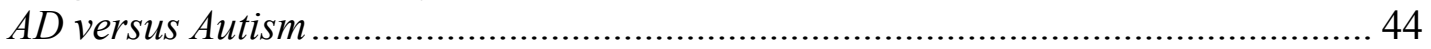

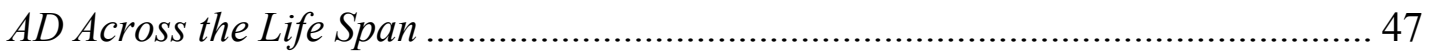

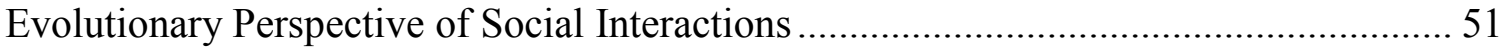

Social Deficit and Cognitive Deficit Hypotheses ........................................................... 54

Theories that Support the Social Deficit Hypothesis........................................................ 55

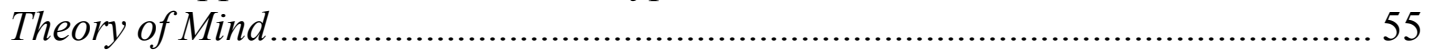

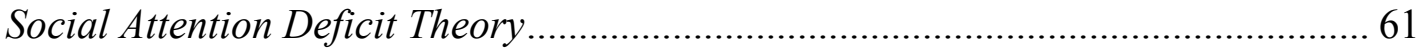

Theories that Support the Cognitive Deficit Hypothesis ....................................................6 66

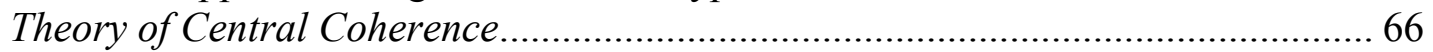


Right Hemispheric Deficit Theory and Neurological Underpinnings ..................... 70

Linking Core Hypotheses to Working Memory ............................................................... 75

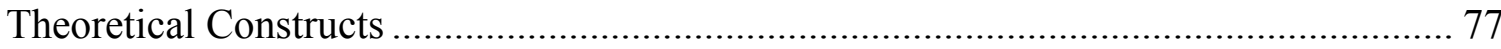

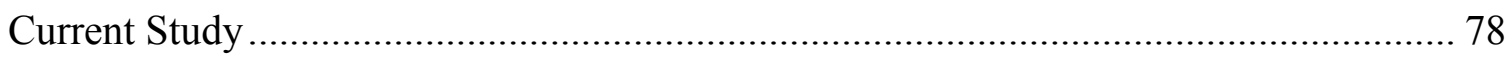

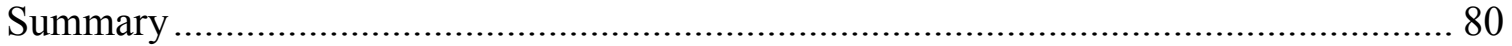

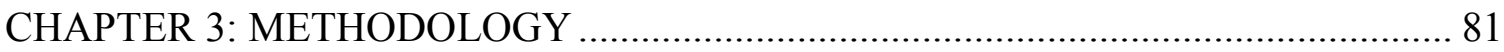

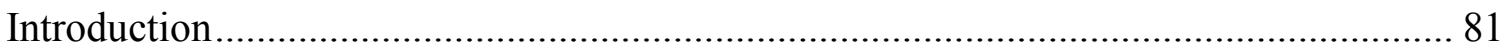

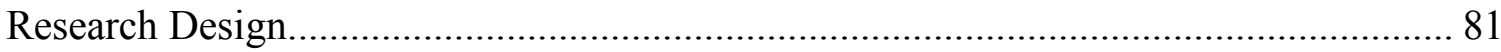

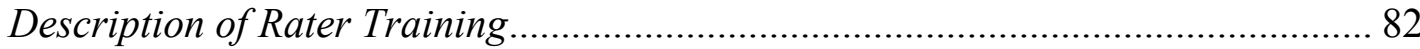

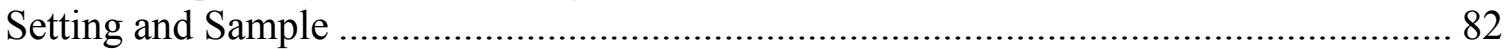

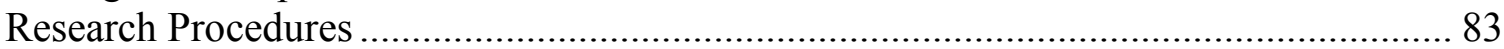

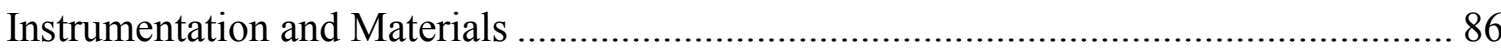

The Gilliam Asperger's Disorder Scale (GADS) ..................................................... 86

The Wechsler Intelligence Test for Children-IV (WISC-IV) ................................. 87

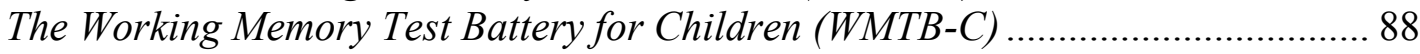

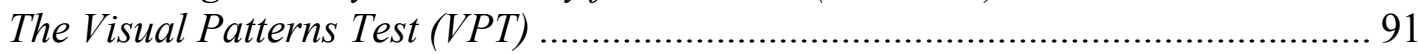

Diagnosis and Assessment of Asperger's Disorder ...................................................... 92

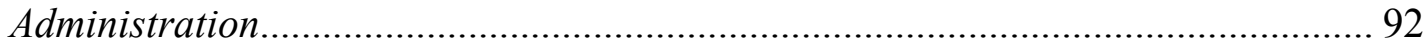

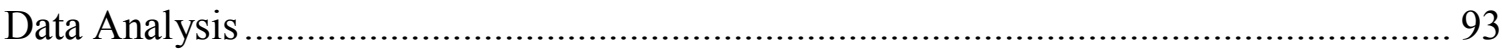

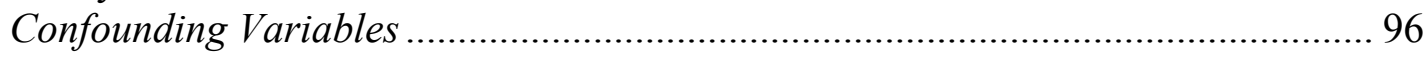

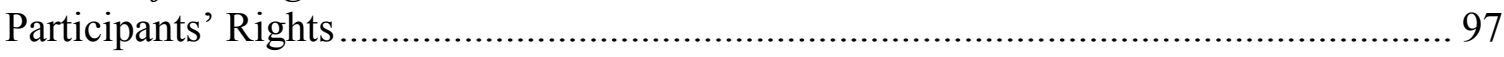

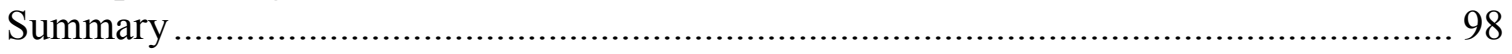

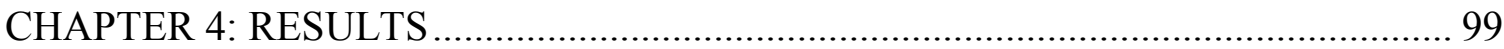

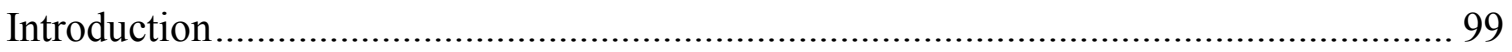

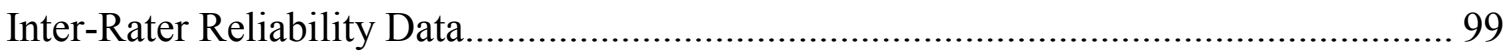

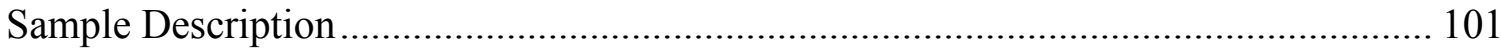

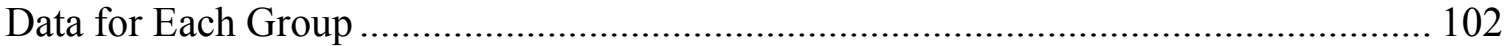

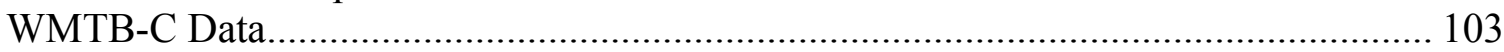

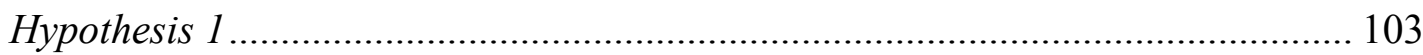

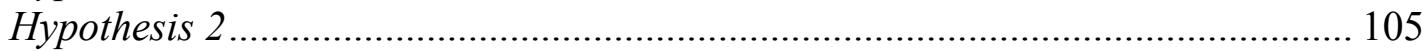

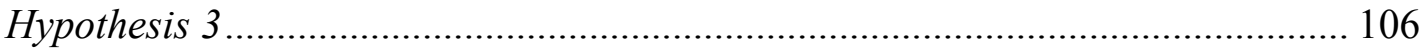

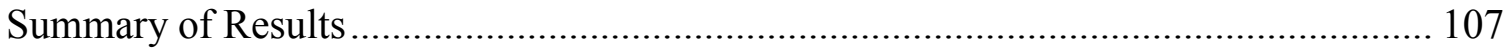

CHAPTER 5: SUMMARY, CONCLUSIONS, AND RECOMMENDATIONS .......... 109

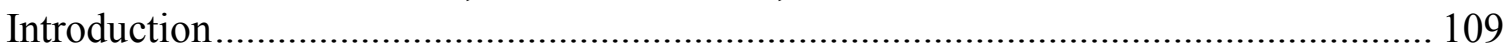

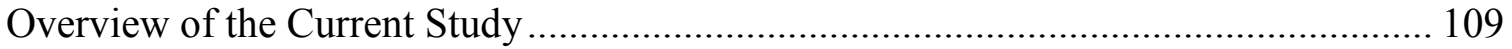

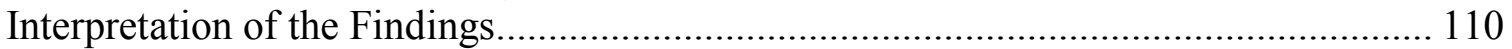

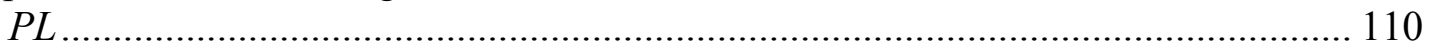

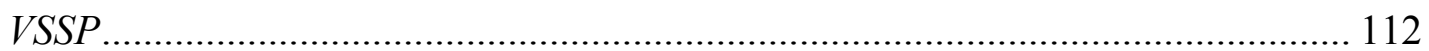

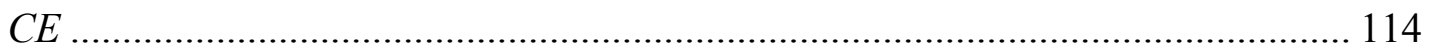

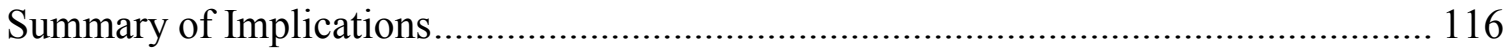

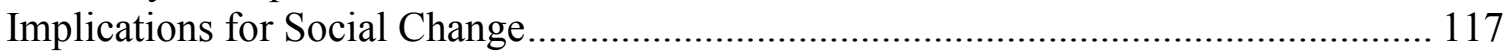




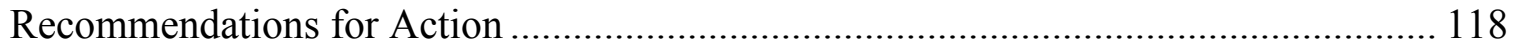

Implications for Future Research........................................................................ 119

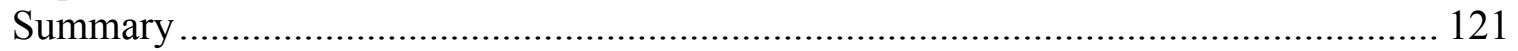

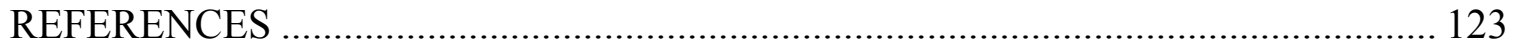

APPENDIX A: Research Assistant Confidentiality Agreement ……………………..... 137

APPENDIX B: Solicitation of Participants ........................................................... 138

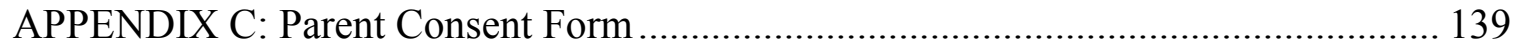

APPENDIX D: Child Consent Form 142

APPENDIX E: General Information Form ............................................................ 143

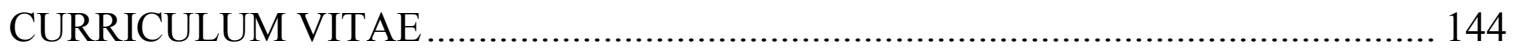




\section{LIST OF TABLES}

Table 1: Inter-rater Reliability for AD Training .......................................................... 100

Table 2: Means and Standard Deviations of Select Sample Descriptors for Group A and

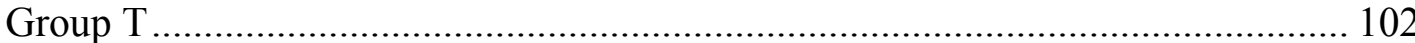

Table 3: ANOVA Testing PL Scores between Group A and Group T............................ 104

Table 4: ANOVA Testing VSSP Scores between Group A and Group T....................... 106

Table 5: ANOVA Testing CE Scores between Group A and Group T ........................... 107

Table 6: PL, VSSP, and CE Score Means, Standard Deviations, and Effect Sizes for

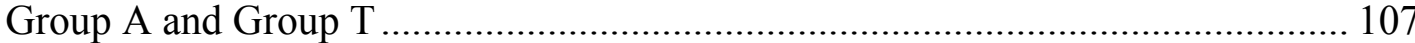




\title{
CHAPTER 1: INTRODUCTION TO THE STUDY
}

\author{
Introduction
}

Children who meet the diagnostic criteria for Asperger's disorder (AD) are characterized by their inability to develop social competence or skills, resulting in the inability to socially relate to others (American Psychiatric Association [APA], 2000; Carrington, Templeton, \& Papinczak, 2003; Gilliam, 2001; Gutstein \& Whitney, 2002). Although some children with $\mathrm{AD}$ have normal language development, they demonstrate a significant inability to utilize nonverbal social cues, regulate verbal and nonverbal social interaction, or engage in social or emotional reciprocity with others (Bauer, 1996; Carter, Meckes, Pritchard, Swensen, \& Wittman, 2004; Gutstein \& Whitney; Molloy \& Vasil, 2002). AD was identified in the 1940s by Hans Asperger; however, not until 1994 was the disorder listed as an independent diagnosis under the diagnostic category of Pervasive Developmental Disorders (PDDs) in the Diagnostic and Statistical Manual of Mental Disorders (DSM-IV-TR; APA). The etiology of AD remains unknown and unclear (Volkmar, Lord, Bailey, Schultz, \& Klin, 2004); public awareness of AD is limited (Attwood, 2004); and effective treatment interventions for AD are understudied (Little, 2003).

Social interactions require that individuals perform the complex task of processing verbal information (e.g., comprehending direct commands and listening to and comprehending words and the global content of verbal conversation) while simultaneously processing nonverbal information such as eye contact/gaze, hand gestures, body postures, and facial expressions. Social competence and the ability to 
perform complex social skill tasks such as reciprocal conversation and pragmatic communication skills are deficits that individuals with AD demonstrate (Gilchrist et al., 2001).

To understand the information-processing (IP) deficits of individuals with AD, researchers have generated two hypotheses, namely, the social deficit hypothesis and the cognitive deficit hypothesis, which has resulted in the development of four primary theories. The social deficit hypothesis is supported by the theory of mind (Baron-Cohen \& Hammer, 1997; Baron-Cohen, Jolliffe, Mortimore, \& Robertson, 1997; Frith \& Happé, 1999; Happé \& Loth, 2002; Lawson, 2003) and the social attention deficit theory (Clark, Feehan, Tinline \& Vostanis, 1999; Dawson, Meltzoff, Osterling, Rinaldi, \& Brown, 1998; Dawson et al., 2004). This hypothesis explores IP deficits in attention and awareness of social cues; ability to infer the thoughts and mental state of another individual (Baron-Cohen \& Hammer); and ability to comprehend social information and social cues (Dawson et al., 2004).

The cognitive deficit hypothesis, supported by the theory of central coherence (Frith, 1989; Morgan, Mayberry, \& Durkin, 2003) and the right hemispheric theory (Craig \& Baron-Cohen, 1999; Rourke et al., 2002), explores IP deficits in the cognitive skills necessary for social interactions: ability to organize and differentiate whole/part relationships; amalgamate and analyze details of the social interaction; translate details into a higher level of meaning (Frith); and understand visual-spatial perception, visual memory, nonverbal concept formation and higher orders of reasoning (Craig \& BaronCohen; Rourke et al.). 
Both hypotheses have proposed that deficits in IP result in the failure to understand and respond to social situations. Although each hypothesis has been supported by empirical research, neither has fully explained the underlying etiology, symptomatology, or complex nature of the significant social skill deficits in individuals with AD (Gunter, Ghaziuddin \& Ellis, 2002; Volkmar et al., 2004). Instead, each hypothesis has offered a partial explanation for the inability of individuals with AD to understand and utilize social cues; develop social competence (Gutstein \& Whitney, 2002); and appropriately socialize with peers (Falk-Ross, Iverson, \& Gilbert, 2004). To understand the underlying causes of IP deficits and their relationship to the complex tasks inherent in social interaction, areas that address specific IP skills, such as working memory, need to be explored.

The concept of working memory evolved from early studies of short-term memory, which was viewed as a unitary system of memory known as iconic memory (Neisser, 1967) that transferred information linearly into long-term storage (Gegenfurtner \& Sperling, 1993). Experiments assessed short-term memory capacity by using traditional short-term memory tasks or simple span tasks (Bayliss, Jarrold, Gunn, \& Baddeley, 2003; DiLollo \& Dixon, 1988).

As research continued, iconic memory was found to be too simplistic (Daneman \& Carpenter, 1980). The unitary system was revised to become two separate memory systems, namely, primary (short term) and secondary (long term; Gathercole, 1998), that demanded a higher level of cognition to simultaneously manipulate information. This concept of two memory systems led to the development of the resource-sharing (RS) 
model (Daneman \& Carpenter) and the multiple resource-sharing (MRS) model of working memory (Baddeley, 1966; Gathercole; Loisy \& Roulin, 2003; Shulman, 1971). The MRS model defined working memory as a limited-capacity system responsible for identifying, coding, storing, manipulating, and retrieving information relevant to the current cognitive task (Baddeley, 1996).

Working memory capacity is the ability to interpret, remember, and store multiple types of information (i.e., verbal, visual, and spatial) pertaining to the current task while simultaneously organizing, maintaining, and retrieving already learned information to perform a complex task (Baddeley, 1996, 2001, 2003). Information processed in working memory is verbal and nonverbal. Deficits in working memory capacity directly affect complex task performance (Gathercole \& Pickering, 2001). Working memory research has established that complex tasks such as reading and arithmetic are negatively affected by deficits in working memory (Baddeley, Gathercole, \& Papagno, 1998; Bull, Johnston, \& Roy, 1999); however, research addressing how the complex task of social interactions relates to working memory capacity remains understudied.

\section{Purpose of the Study}

The purpose of this study was to discover any significant differences in IP capacity between individuals with $\mathrm{AD}$ and typically developing, same-age peers in verbal, visual, and spatial working memory capacities. Children with AD have difficulty processing nonverbal and emotional information within a social context (Barnhill, Hagiwara, Myles, \& Simpson, 2000; Ghaziuddin \& Mountain-Kimchi, 2004; Gunter et al., 2002; Safran, Safran, \& Ellis, 2003). The social deficit and cognitive deficit 
hypotheses do not fully explain the specific IP deficits that may contribute to the inability of individuals to engage in the complex task demands of social interactions.

The consequence of not isolating the specific type of IP deficit related to working memory capacity has resulted in vague assumptions about the deficits specific to children who show symptoms of AD. By isolating and assessing IP skills and deficits, differences in how individuals with $\mathrm{AD}$ process information in comparison to their typically developing, same-age peers can be better understood. This area of study may have been neglected because of the lack of a comprehensive, reliable, and valid instrument to assess the working memory capacity of children (Pickering \& Gathercole, 2001). In 2001, the Working Memory Test Battery for Children (WMTB-C) became available to assess the verbal, visual-spatial, and complex working memory capacity of children (Pickering, 2001b; Gathercole \& Pickering, 2001).

If IP deficits in the working memory capacity of AD individuals can be identified, a clearer understanding of deficits in the perception, memory, manipulation, and retrieval of verbal, visual, and spatial information during the performance of complex tasks will be achieved. Working memory capacity may relate to the ability to perform the complex tasks inherent in the demands of social interactions (Bayliss et al., 2003).

\section{Statement of the Problem}

Researchers have not adequately addressed how IP deficits specific to working memory affect the way in which individuals with $\mathrm{AD}$ interact socially and perform the complex IP tasks inherent in social interactions. Although the four main theories (i.e., theory of mind [TOM], social attention, central coherence, and right hemisphere deficit) 
underlying the social and cognitive hypotheses accept that individuals with AD display social and cognitive IP deficits, none of them has conclusively identified the specific IP deficits underlying social comprehension problems. The theories identify complex tasks and IP skills that contribute to successful social behavior (i.e., TOM skills, social cue attention skills, nonverbal cue perception skills and central coherence skills), but they do not address IP deficits in processing information (i.e., verbal, visual-spatial, and complex) specific to working memory. By isolating these IP deficits, researchers can more readily assess their impact. What remains unknown is how specific IP deficits related to working memory contribute to the inability of individuals with AD to engage in the complex task demands of social interactions.

\section{Nature of the Study}

This study was a survey designed to ascertain a possible relationship between working memory capacity and level of social competence. Sixty boys between the ages of 11 and 15 who met the criteria for the study participated. Thirty boys previously diagnosed with AD were placed in the Asperger's Disorder group (A), and 30 boys not diagnosed with $\mathrm{AD}$ or any other disorders were placed in the Typical Social Competence group (T). The WMTB-C (Pickering \& Gathercole, 2001) assessed the working memory capacity of these participants. The independent variable (IV) was level of social competence; the dependent variables (DVs) were verbal, visual-spatial, and complex working memory capacity. The procedure was designed to isolate the IP deficits specific to verbal, visual-spatial, and higher level working memory to identify a relationship of 
the isolated "types" of IP deficits between the two groups. The resulting data were analyzed using a set of three ANOVAs, as appropriate.

Research Hypotheses

Null Hypothesis 1: There are no differences in verbal working memory functioning between children with $\mathrm{AD}$ and their typically developing, same-age peers, based on the WMTB-C.

Alternative Hypothesis 1: Children with AD demonstrate a lower level of verbal working memory capacity than their typically developing, same-age peers, based, on the WMTB-C.

Verbal working memory, identified as the Phonological Loop (PL), is the ability to process, hold, and store verbal information in terms of sound structure and spoken language.

Null Hypothesis 2: There are no differences between visual-spatial working memory capacity of children with AD and their typically developing, same-age peers, based on the WMTB-C.

Alternative Hypothesis 2: Children with AD demonstrate a lower level of visual-spatial working memory capacity than their typically developing, same-age peers, based on the WMTB-C.

Visual-Spatial working memory, identified as the Visual-Spatial Sketch Pad (VSSP), is the capacity to store nonverbal information that has either visual (color, shape) or spatial (movement, relative location) characteristics. 
Null Hypothesis 3: There is no difference between complex working memory capacity of children with AD than their typically developing, same-age peers, based on the WMTB-C.

Alternative Hypothesis 3: Children with AD demonstrate a lower capacity of complex working memory capacity than their typically developing, same-age peers, based on the WMTB-C.

Complex working memory, identified as the Central Executive (CE), is responsible for planning and coordinating the flow of information and retrieving it from long-term memory.

\section{Definitions of Terms}

Asperger's Disorder (AD): A subcategory of PDDs in the DSM-IV-TR (APA, 2000). The essential features of this disorder are severe and sustained impairment of social interactions; restricted, repetitive, and stereotyped patterns of behaviors and interests; and the inability to utilize simultaneous nonverbal information such as eye contact, gesture, facial expression, and eye gaze in the context of social relationships (APA; Koning \& MaGill-Evans, 2001).

Central Executive (CE): A domain-general system responsible for controlling the flow of information throughout the working memory (Bull et al., 1999). In this study, CE capacity is measured by the Listening Recall, Counting Recall, and Backward Digit Recall subtests of the WMTB-C (Pickering \& Gathercole, 2001).

Complex Span Tasks: Distinguished from traditional short-term memory tasks in that multiple cognitive activities are performed simultaneously (Bayliss et al.; Duff \& 
Logie). Complex span tasks measure the dynamic processing and storage capacity of working memory during task performance rather than the passive recall of items required by simple span tasks.

Episodic Buffer (EB): A domain-specific subcomponent of working memory defined by the MRS model. The EB temporarily stores information that has been coded and organized by the CE (Baddeley, 2003).

Gilliam Asperger's Disorder Scale (GADS): A 32-item scale that includes 4 subscales: social interaction, restricted patterns of behavior, cognitive patterns, and pragmatic skills. Raw subscale scores are converted into $\mathrm{T}$ scores to yield a total score known as the Asperger's Disorder Quotient (ADQ; Gilliam, 2001).

Phonological Loop (PL): A domain-specific subcomponent of working memory defined by the MRS model. The role of the PL is to store and temporarily retain verbal information (acoustic or speech based) through two subcomponents of the PL: phonological store and articulatory rehearsal process (Gathercole, Pickering, Knight, \& Stegmann, 2003; Pickering, Gathercole, Hall, \& Lloyd, 2001). In the present study, this variable is PL capacity as measured by the Digit Recall, Word List Matching, Word List Recall, and Nonword List Recall subtests of the WMTB-C.

Simple Span Tasks: Traditional short-term memory tasks in which passive storage mechanisms of memory are assessed by tasks such as digit span and word span. The individual is asked to remember one type of information presented in the recent past without simultaneously performing another task (Baddeley, 1996; Bayliss et al., 2003; Duff \& Logie, 2001). 
Visual Patterns Test (VPT): A test of short-term visual recall intended to measure only the visual aspects of nonverbal short-term memory (Della Sala, Gray, Baddeley, \& Wilson, 1997). In the present study, scores from this test were utilized as a subtest for VSSP and integrated into the composite score of the individual's VSSP capacity, as indicated in the WMTB-C manual (Pickering \& Gathercole).

Visual-Spatial Sketch Pad (VSSP): A domain-specific subcomponent of working memory defined by the MRS model. The VSSP stores and temporarily retains visual and spatial information in working memory (Gathercole et al.; Pickering et al.). In the present study, this variable is VSSP capacity as measured by the Block Recall and Mazes Memory subtests of the WMTB-C.

Working Memory: The limited-capacity system responsible for identifying, coding, storing, manipulating, and retrieving information relevant to the individual's current cognitive task (Baddeley, 1996).

Working Memory Test Battery for Children (WMTB-C): A battery that includes 9 subtests designed to assess short-term working memory capacity in children. This assessment is theoretically related to the working memory capacity levels defined by the tripartite structure of the MRS model (Gathercole \& Pickering, 2001).

Assumptions of the Study

1. The WMTB-C (Pickering \& Gathercole, 2001) is a new instrument that assesses the working memory capacity of children between the ages of 5.5 and 15.5 . It yields three scores: PL, VSSP, and CE. The researcher assumed that this instrument had not been 
utilized previously with any of the participants in the study; therefore, bias from instrument exposure was not an issue.

2. The sample was appropriate for the study.

3. The researcher followed administration and scoring requirements for the various instruments used in this study (WMTB-C, the Wechsler Intelligence Scale for Children [WISC III and IV], and Gilliam's Asperger's Disorder Scale [GADS]) and did not skew the results.

4. The data derived from study identified a correlative factor between the individuals who met the criteria for $\mathrm{AD}$ and working memory capacity, but it was not interpreted as evidence of a causative factor of IP deficits.

\section{Ethical Issues}

Permission to conduct the study was granted by the Committee on Ethical Standards in Research for the Institutional Review Board (IRB) at Walden University before initiating this study. Permission was then obtained from two high schools in northern and central Ohio to solicit volunteers from each school. Permission for them to participate in the study was obtained by their parents/guardians. All participants were informed of their legal rights in terms of understanding the nature and purpose of the study; their rights to consent to participate; and their right to withdrawal at any time without penalty, consequence, or harm. Parents/Guardians were informed that the testing sessions would not exceed 2 hours. If more than 2 hours was needed to complete an assessment, arrangements for an additional session were be made. There was no risk associated with participating in this study. 
Limitations of the Study

1. The working memory capacity was based on scores derived from the WMTB-C. Thus, a definitive cognitive or emotional diagnosis was not rendered for the participants.

2. The GADS was used to determine if the individual met the criteria for inclusion in Group A (i.e., characteristic of AD) or Group T (i.e., typically developing, same-age peers). All participants eligible for the AD group had a previous diagnosis of AD. The GADS was used to verify either low or typical social competence skills, not to diagnose a medical or mental health disorder.

3. This study sample comprised male participants between ages 11.0 to 15.5 years. The target population for specific participants was not to suggest that AD symptoms do not occur in females or other males younger than 11.0 or older than 15.5.

4. The researcher administered an exploratory study in her professional field. She was not involved with the participants in any professional or personal manner other than in her capacity as a researcher. Therefore, a conflict of interest did not bias the results or the rights of the volunteer participants.

\section{Significance of the Study}

The study contributed to the scientific knowledge of the working memory capacity of children by investigating the relationship between IP deficits and working memory capacity identified specific IP deficits of children who lack social competence and social skills. Understanding how individuals with AD process information and become aware of, perceive, code, remember, interpret, integrate, comprehend, and manipulate nonverbal and verbal cues related to working memory capacity will increase 
knowledge of specific IP capacities related to social skills and social competence. Individuals with $\mathrm{AD}$ who acquire social skills needed to interact with peers may decrease experiences of isolation, dependence, and peer rejection. Instead, with the ability to process information needed to for appropriate social interactions, they may participate in social peer groups, experience intimacy and social acceptance, and achieve independence. Social interaction skills and social competence are critical to the ability to succeed in academic, work, social, family, and emotional relationships (Denham et al., 2003). As well, treatment and interventions can be coordinated to target natural developmental timelines with regard to when working memory skills emerge.

Summary

The four major theories derived from the social and cognitive deficit hypotheses are the theory of mind, the social attention deficit theory, the theory of central coherence, and the right hemispheric hypothesis theory. The cognitive and social hypotheses concur that individuals with AD display cognitive and/or social IP deficits; however, neither has generated conclusive evidence about the specific deficits that underlie the social comprehension problems (Little, 2003), nor has either one addressed the specific IP deficits (verbal, visual, and spatial) related to working memory capacity. The study focused on specific IP deficits in processing and interpreting nonverbal, verbal, and spatial information inherent in social relationships as they relate to working memory capacity. The results of this research will provide valuable information about the specific IP deficits in individuals who meet the DSM-IV-TR (APA, 2000) criteria for AD. 
Chapter 2 provides a comprehensive literature review on working memory, cognitive and social deficit hypotheses related to $\mathrm{AD}$, and the four theories that support each hypothesis. Working memory related to IP and social deficits characteristic of individuals with $\mathrm{AD}$ is discussed. Chapter 3 describes the research design, methodology, data collection, and analysis procedures. Chapter 4 presents the results of the data. Chapter 5 summarizes the study and discusses the implications of future research. 


\section{CHAPTER 2: LITERATURE REVIEW}

\section{Introduction}

A comprehensive literature research was undertaken more than 3.5 years (20022005) prior to implementing the study. The databases included BIOMED, CINAHL, IUCAT, NIH/NIM MEDLINE, PsycINFO, and PubMed. The major keywords and abbreviations were Asperger's disorder, autism, CE, executive functioning, frontal lobe dysfunction, IP, neurobiology, neuropsychology, short-term memory, social skills, PL, VSSP, and CE. The retrieval sources included the library services of Akron University, Cleveland State University, Indiana University, and Walden University.

This chapter begins with a discussion of the history and development of working memory theory and working memory models: the resource sharing (RS) model and the multiple resource-sharing (MRS) model. The discussion continues by relating IP skills to specific types of working memory processes and how working memory capacity influences social interactions. The literature review summarizes the essential features of $\mathrm{AD}$, assessment, and the two major hypotheses (social deficit and cognitive deficit) underlying the social deficits characteristic of AD. The literature search also examines the theories in terms of the etiology, limitations, and relationship to working memory in regard to IP inherent in social relationships. The chapter concludes with a discussion of how IP deficits relate to the current study. 
History and Development of Working Memory Theory

\section{Short-Term Memory}

The traditional model of human memory is derived from experiments in the $1950 \mathrm{~s}$ and 1960 s that focused on testing short-term, or iconic, memory. Iconic memory is a term coined by Niesser (1967) describing how a visual image is stored either partially or wholly or decays in seconds after the individual is cued to remember the image (Gegenfurtner \& Sperling, 1993). Iconic memory is a measure of how many items and their physical properties (size, shape, color) an individual can recall immediately after viewing them. Iconic memory is a photographic-like image that remains for "a few hundred milliseconds after the inducing of a stimulus had been turned off' (DiLollo \& Dixon, 1988, p. 671). Iconic memory experiments have utilized quantitative methods to measure the capacity of short-term memory to visually search, attend to, and recall "icons" introduced in the very recent past (Gegenfurtner \& Sperling; Goldman-Rakic, 1996).

Iconic memory testing has focused on the productive capacity of human shortterm memory by using serial recall techniques. Sperling (as cited in Goldman-Rakic, 1996) initiated the first short-term memory experiments measuring the number of items an individual can remember from serial recall. Two popular serial recall techniques are free recall and differential decoding. The free recall technique requires the individual to recall as many words as possible in any order from a list of unrelated words. The differential decoding technique requires the individual to recall as many words as 
possible in the order of presentation from a list of unrelated words (Goldman-Rakic). Experiments also may include the recall of visual images.

Entwisle and Higgins (1973) conducted their experiment on children in Grade 1. They rapidly showed the children 40 black-and-white slides of landscapes and cityscapes. They found that the children remembered the visual images for hours and then weeks. They repeated the experiment on children in Grade 2 and compared iconic memory capacity between visual and verbal materials. They noted that the children in both grades remembered visual images far better than verbal material. Experiments such as this began to question the linear structure of the short-term memory system.

The linear structure of iconic memory assumes that memories are stored in a unitary structure that identifies, attends to and remembers a set of objects. However, the unitary system of short-term memory was challenged by studies that contradicted the linear system by identifying two separate components: primary and secondary memory. The primary memory component holds information temporarily: the secondary memory component holds information permanently (Baddeley, 1996; DiLollo \& Dixon, 1992, 1998; Gathercole, 1998; Shulman, 1971). As research continued, the concept of multiple short-term memory components was integrated and new models of short-term memory were developed: the modal model and the working memory model.

The modal model integrates primary (i.e., short-term) and secondary (i.e., longterm) memory and identified functions of encoding, maintenance, and retrieval (Healy \& McNamara, 1996). The modal model assumes that information is processed through three linear systems: (a) perception and sensory, (b) short-term memory, and (c) long-term 
memory (Baddeley, 20002b). Although the modal model gained support, it was criticized for being too simplistic, linear, and passive, as well as for deemphasizing the storage and processing capacity of memory involved in encoding, maintaining, and retrieving information (Baddeley; Dutke \& Stober, 2001). The limitations and criticisms of the modal model led to the development of the working memory model, which emphasized nonlinear subsystems of short- and long-term memory and the functions of storage and processing capacities (Healy \& McNamara; Kane et al., 2004).

\section{Working Memory Model}

The term working memory is a theoretical construct "widely used to refer to a mental workplace in which information can be temporarily stored and manipulated in order to support ongoing activities" (Gathercole \& Pickering, 2001, p. 178). The function and capacity of working memory are determined by the ability to identify, code, store, manipulate, and retrieve information relevant to the current cognitive task (Baddeley, 1996). Working memory theory encompasses how the capacity to encode and remember various forms of information influences the ability to reason, comprehend, perform complex tasks, and simultaneously process information at that moment and over time (Baddeley, 1996, 2002a, 2003; Marton \& Schwartz, 2003).

The limitations of early short-term memory models prompted the investigation of a more satisfactory explanation of how information is actively processed rather than passively stored and how multiple and independent systems of coding information contribute to specific IP tasks (Gathercole, 1998). Traditional short-term memory tasks are simple span tasks: The individual is asked to remember one type of information 
without performing another task simultaneously (Loisy \& Roulin, 2003). Simple span task assessments have provided strong evidence that traditional short-term memory techniques are inadequate and do not consider that the individual utilizes higher cognitive working memory processes that may be nonlinear (Daneman \& Carpenter, 1980). Simple span tasks such as word span and digit span are tests of passive storage mechanisms. They do not test the dynamic processing and storage capacity of the working memory. Limitations of the linear short-term memory model became evident when assessing errors in short-term visual and verbal memory recall. Errors in verbal shortterm memory task performance were associated with phonological similarities, a verbal function related to the rehearsal and storage processes of the PL. Errors in visual shortterm memory tasks were associated with delay variations and interruptions, a function related to the rehearsal and storage process of the VSSP. These errors suggested that short-term memory capacity is nonlinear, systematically manipulated, and not a passive system dependent on immediate short-term memory (Baddeley, 1996). Linear models of memory cannot explain errors, nor can they explain how an individual can function well in daily life tasks but demonstrate significant short-term memory deficits (Baddeley). This paradox led to the investigation of higher cognitive abilities and working memory capacity by using complex span task analysis (Bayliss et al., 2003). Complex tasks, in addition to simple tasks, differentiated short-term memory and working memory functions. 


\section{Complex Span Tasks of Short-Term Memory}

The main difference between traditional short-term and working memory functions is the use of complex span tasks rather than passive short-term memory (i.e., simple span tasks) to investigate the memory system (Bayliss et al., 2003). Complex span tasks are different from traditional simple span memory tasks because multiple forms of information to be processed simultaneously (Bayliss et al.; Duff \& Logie, 2001). In contrast, traditional simple span memory tasks measure storage capacity only (Bayliss et al.). Complex span tasks require the performance of multiple cognitive activities. For example, a complex span task may require the individual to do mental arithmetic or read words while holding a group of unrelated numbers or digits in memory during the task. After completion of the task, the individual is to recall the first set of numbers or letters stored in short-term memory. Complex span tasks measure online processing and temporary memory load while the individual is simultaneously performing a task requiring the active processing and storage of information (Kane \& Engle, 2000; Shah \& Miyake, 1996; Turner \& Engle, 1989).

Traditional short-term memory tasks such as word span and digit span are tests of passive storage mechanisms. They do not test the dynamic processing and storage capacity of the working memory (Baddeley, 1996; Bayliss et al., 2003; Daneman \& Carpenter, 1980; Duff \& Logie, 2001; Gathercole, Pickering, Ambridge, \& Wearing, 2004). The concept of working memory had been explored in higher cognitive functioning such as reading comprehension, mathematical abilities, reasoning, problem solving, and complex task performance (Daneman \& Carpenter; Just \& Carpenter, 1992; 
Hitch, Towse, \& Hutton, 2001). The use of complex span tasks led to the development of the RS model and the MRS model of working memory.

\section{RS Model of Working Memory}

Daneman and Carpenter (1980) developed the RS model of working memory, which incorporates the concept of two separate memory systems rather than a unitary system of short-term memory, thereby supporting the contention that multiple short-term memory systems share a common resource pool (Baddeley, 1966; Gathercole, 1998; Loisy \& Roulin, 2003; Shulman, 1971). When the pool of working memory resources is depleted, the working memory capacity declines during task performance, and the individual is unable to continue processing or storing information pertaining to that task (Duff \& Logie, 2001).

Initially, Daneman and Carpenter $(1980,1983)$ employed the RS model to test the relationship between reading comprehension and PL by utilizing complex span tasks rather than traditional simple span tasks of short-term memory. They concluded that individuals who read poorly have a smaller capacity to remember what they have read because they allocate more resources to reading the words rather than processing the meaning and content of the information. The RS model asserts that the multiple cognitive tasks of reading the words, remembering the content, and comprehending the material are supported by a shared pool of working memory resources. Individuals with fewer resources comprehend less content; therefore, their PL capacity is lower (Daneman \& Carpenter, 1980, 1983). The verbal complex reading span tasks conducted by Daneman and Carpenter $(1980,1983)$ supported the RS model's position that working memory 
resources are shared and are domain general. However, complex span tasks that investigated visual and spatial information along the same lines as PL contradicted this view (Duff \& Logie, 2001). Duff and Logie found that experiments involving complex span tasks done by children have not supported a trade-off of shared pool resources that compete.

Towse, Hitch, and Hutton (1998) investigated the RS model's premise that the retention and processing systems of working memory are domain general and dependent on a shared pool of resources. They conducted experiments on 67 children between ages 6 and 11 that tested memory decay, processing duration, and processing accuracy through counting span, operation span, and reading span tasks. Towse et al. found that the processing speed of the children was not compromised while manipulating complex span tasks utilizing concurrent storage and processing functions of working memory.

A number of researchers have supported Towse et al.'s (1998) findings and have criticized the premise of the RS model (Daneman \& Merikle, 1997; Engle, Kane, \& Tuholski, 1999). The most compelling criticisms of the RS model relate to evidence supporting the interplay between multiple working memory components (Daneman \& Merikle; Engle et al.), as opposed to a general pool of shared resources of the model's linear view. Additionally, contradictory evidence has identified processing speed and accuracy skills as independent of a general RS pool in that during the performance of complex tasks, speed and accuracy are not related to span score results (Conway \& Engle, 1996; Towse et al., 1998, 2000). 
Results from these studies were in direct contrast to the RS model, which suggests that processing speed decreases as demands for working memory processing and storage increase because working memory shares a pool of resources. Previous studies have found that processing speeds are not affected. Participants were able to use a strategy to hold information and switch tasks without disrupting processing speed, suggesting that processing and storage are not domain general and do not compete simultaneously for a resource pool of working memory (Conway \& Engle, 1996; Towse et al., 1998, 2000).

\section{MRS Model of Working Memory}

The MRS model of working memory developed by Baddeley and Hitch (1974) proposed that working memory resources are domain specific, active, and nonlinear (Baddeley, 1996; Bayliss et al., 2003; Duff \& Logie, 2001). The MRS model identified two highly specialized, independent, and domain-specific subsystems supported by a shared central system that is domain general (Bayliss et al.; Gathercole, 1998; Gathercole et al., 2004). Each component of working memory works together but functions nonlinearly and independently.

The operational definition of working memory capacity is the ability to process information (verbal, spatial, visual); maintain relevant information of the current task or interaction online (emotional, cognitive, or social); and simultaneously interpret, store, and retrieve already learned information to use in response to the current situation or complex task (Baddeley, 2001). The conceptual definition of working memory capacity is more complex. Although there is no universal agreement on the definition of working memory (Barrett, Tugade, \& Engle, 2004), the concept relates to the interplay of the four 
principal components of the working memory. Conceptually, individual differences in the automatic processing capacities of working memory determine one's ability to perform complex tasks. Performance is dependent upon one's automatic ability to keep some information of the current task active; manipulate parts of the task (mentally rotate, count, read, compute, etc.); shift attention periodically; retrieve already stored information; and store new information for later recall (Baddeley, 2002b, 2003; Baddeley \& Hitch, 1974). The MRS model of working memory identified the gateway of a limitedcapacity system that conceptually allows the individual to do this.

\section{Components of the MRS Model}

The MRS model has four components: the Central Executive (CE); the Phonological Loop (PL); the Visual-Spatial Sketch Pad (VSSP); and the model's most recent addition, the episodic buffer $(\mathrm{EB})$. This tripartite model illustrates how information is perceived, coded, stored, and manipulated through short-term and long-term memory to allow the individual to perform complex cognitive tasks. Task performance related to working memory includes storing and retrieving visual, verbal, and spatial information (Baddeley \& Logie, 1999, Pickering, 2001a). The CE and the EB are domain-general components that support multiple complex activities while regulating the information of the subsystems (Gathercole \& Pickering, 2001). The EB is a subsystem that binds information that has been processed and organized through the CE (Baddeley, 2003). The PL and the VSSP are independent, domain-specific, specialized subsystems that process verbal and visual-spatial information (Baddeley, 2002b; Bull et al., 1999; Gathercole \& Pickering). They are responsible for processing information that requires rehearsal so that 
the information is remembered (Park et al., 2002). The subsystems function together by way of the CE.

\section{The Central Executive}

The CE is the domain-general system (Pickering, 2001b) that is responsible for controlling the function and flow of information from the three domain-specific subsystems: the PL, the VSSP, and the EB (Baddeley, 2003; Bull et al., 1999). The CE is a complex system that has multiple roles: inhibiting irrelevant information from interfering with working memory capacity, updating information in working memory, switching between tasks while evaluating new strategies, and maintaining relevant problem-solving information of both tasks (Bull \& Scerif, 2001).

Functions of the CE overlap with those of the executive functioning in terms of organizing material, planning skills, initiating problem-solving methods, and sustaining attention and generalized memory (Kibby, Marks, Morgan, \& Long, 2004; Miyake, Friedman, Rettinger, Shah, \& Hegarty, 2001). Studies in developmental neuropsychology have investigated the executive functioning skills and working memory skills of the CE. Researchers have found that both are complex structures that perform similar functions activated by the frontal lobes (Bull et al., 1999; Welch, Pennington, \& Groisser, 1991). Children may have varying levels of executive functioning and CE skills in working memory that directly relate to the development of the frontal lobes (Case, 1992; Chelune \& Baer, 1986). By age 7, individual differences may be significant because of the variability of frontal lobe development, which occurs between ages 7 and 10 (Case; Chelune \& Baer). 
The role of the CE in working memory is to coordinate the retrieval of information stored in long-term memory integrated into the EB while controlling the action, planning, and scheduling of multiple cognitive activities of the current task (Baddeley, 2002a; Gathercole \& Pickering, 2001). The CE regulates verbal information to and from the PL and visual-spatial information to and from the VSSP as information is rehearsed and stored in long-term memory within the subsystem (Mueller, Seymour, Kieras, \& Meyer, 2003). "In terms of processing information, the central executive is driven by limited-capacity resources that can be used flexibly depending on the demands placed on it" (Kibby et al., 2004, p. 350).

\section{The Phonological Loop}

The PL temporarily retains acoustic or speech-based information in phonological form through two subcomponents: the phonological store and an articulatory rehearsal process (Gathercole et al., 2003; Pickering et al., 2001). The MRS model identifies these subsystems as two separate independent mechanisms of the PL that process verbal information (Awh et al., 1996). Behavioral and neuropsychological evidence has supported the model's premise that the articulatory rehearsal process and phonological store are separate (Awh et al.). Positron emission tomography (PET) research has found that separate anatomical areas mediate the two independent PL mechanisms. The posterior parietal regions of the brain are activated during the storage of verbal information; the anterior part of the brain is activated during speech rehearsal of information (Awh et al.). This neurobiological finding is congruent with the model in that remembering verbal information is a function of two independent PL processes, namely, 
one that temporarily stores information and a separate articulatory mechanism that rehearses this information so that it will not decay within seconds (Baddeley, 2003).

Verbal information introduced to the individual is held as a memory trace for 2 or 3 seconds. After that, the information is subject to rapid decay, which occurs unless a subvocal rehearsal process (i.e., articulatory rehearsal) rehearses, repeats, and refreshes the content of the phonological store (Awh et al., 1996). Subvocal rehearsal of the information is a processing component of PL. It allows the separate systems of rehearsal and storage to preserve and store the information held in the temporary memory trace store of the PL. For example, if an individual is asked to remember a 7-digit phone number, he or she will remember it in the phonological store of short-term memory for only seconds, unless the individual repeats the number several times. This rehearsal process, known as articulatory rehearsal, is the mechanism that allows information to be stored into short-term memory and eventually into long-term memory.

Storage of information in the PL is acoustical in nature. Acoustical storage codes the sensory and physical forms of speech and is highly vulnerable to interference when subsequent speech material is simultaneously introduced (Gathercole, 1999). The individual needs to develop and utilize an articulatory rehearsal mechanism to immediately refresh the content of what is temporarily stored until it is remembered (Gathercole et al., 2003).

Researchers have challenged the mechanism of speech utilized to engage in articulatory rehearsal. Baddeley (1996) contended that articulatory rehearsal requires one to articulate and repeat the word, number, or phrase several times. Caplan, Rochon, and 
Waters (1992) argued against Baddeley's hypothesis and posited that articulatory rehearsal is not based solely on the ability to rehearse information but also on speechplanning ability. Although the speech-planning hypothesis gained much attention, it is not supported in the literature. Instead, researchers have concluded that "rehearsal requires overt or covert articulation of word sequences whose duration affects memory spans significantly" (Mueller et al., 2003, p. 24).

The original assumptions of the PL proposed by Baddeley (2001) have been accepted. Individuals who can construct a speech-motor program, regardless of whether or not they have lost peripheral control of speech musculature, can develop and utilize articulatory rehearsal and store verbal information in the PL (Baddeley \& Wilson, 1985). Individuals who cannot construct a speech-motor program or overtly or covertly articulate speech demonstrate no capacity for articulatory rehearsal (Baddeley \& Wilson; Mueller et al., 2003).

Conners, Atwell, Rosenquist, and Sligh (2001), who examined the decoding abilities of individuals with low intellectual functioning, supported this premise. Children with intellectual disabilities (i.e., Full Scale IQ of less than 70) differed in decoding abilities, not phonemic awareness. Therefore, the ability to rehearse and refresh phonological codes is more significant to learning language and learning how to read rather than to level of intelligence, language ability, and phonemic awareness (Baddeley et al., 1998; Conners et al.). In the present study, individuals who could not articulate speech, had no capacity for articulatory rehearsal, or were unable to refresh the contents of PL were excluded. 
Four main factors affect the PL and determine the effectiveness of the PL in processing and storing information: articulatory suppression, phonological similarity effect, chunking, and word-length effect (Baddeley, 2002; Mueller et al., 2003; Pickering et al., 2001). Articulatory suppression techniques require the individual to perform a verbal-working memory task such as reading silently while simultaneously repeating words aloud that are unrelated to the task. Researchers have used this technique to test if task interference occurs between working memory components. If task interference occurs, the two assigned tasks utilize the same IP component of working memory. Articulatory suppression interferes with PL functioning by preventing one from rehearsing information (Pickering et al.). The PL is dependent on the articulatory rehearsal process; therefore, when rehearsal is prevented, the information is lost or stored poorly, and acoustical storage is prevented (Gathercole, 1998; Service, 1998).

Phonological similarity influences the accuracy of the PL. The phonologicalsimilarity effect occurs when one is trying to remember words that sound similar to one another. Words that are similar sounding are remembered less in the PL than words that are dissimilar (Mueller et al., 2003). Studies on acoustic confusion with words using between two- to nine-letter vocabularies found differences in memory span recall. Conrad and Hull (1964) concluded that if items are similar in sound (e.g., tree, bee, V, see), the short-term memory of these items is poorer than if the item sounds are dissimilar (wind, mile, pack). However, no differences are found in semantic similarity. If words are semantically similar (e.g., big, large, huge, great), immediate serial recall is typically not affected (Baddeley, 1966). This effect provides evidence that the PL, which is based on 
sounds and phonological features, is a domain-specific subcomponent of the working memory (Baddeley). Words that vary in complexity are not a significant predictor of PL capacity after controlling for articulatory duration and word dissimilarity (Mueller et al.).

Chunking is a method of grouping pieces of information together, decreasing the number of items held in memory. Chunking is used with PL tasks as well as VSSP tasks because it allows the individual to quickly identify patterns of verbal or visual information presented and chunk that information into one category to be remembered (Pickering et al., 2001).

Word length effect influences the capacity of PL. When one varies the length of the word by two, three, and four syllables, recall capacities differ more widely between individuals. Experimental research has found that PL can hold a certain number of syllables and that the quantity of recall is determined by how long it takes the individual to articulate the digits in the language rather than how many syllables the individual is asked to remember. Because longer words take more time to articulate, they decay faster because it takes the subvocal/articulatory rehearsal process longer to rehearse the word (Baddeley, 2002b).

\section{The Visual-Spatial Sketch Pad}

The VSSP is capable of retaining and storing visual-spatial information. It identifies and solves problems relating to spatial relationships, visual and spatial patterns, distance, and visual-spatial problems (Baddeley, 2002b). The VSSP is the least understood working memory component of the MRS model in comparison to the CE and the PL (Pickering, 2001b). Initially, the VSSP was viewed as a unitary system until more 
accurate cognitive measures were developed to test this domain-specific component of working memory (Pickering et al., 2001).

Currently, neuropsychological evidence derived from magnetic resonance imaging has identified the VSSP as a multicomponent, not a unitary, system. Separate components are identified for specific visual and spatial IP. Neuropsychological imaging studies have found that visual patterned information is processed through the occipital lobe, spatial information is processed through the parietal regions, and the coordination and control of information is processed through the activation of frontal regions (Smith \& Jonides, 1999). Specifically, the right frontal regions mediate rehearsal processes for spatial information (Smith \& Jonides). Baddeley's (2002a) view was that the CE regulates spatial information by correcting it and rehearsing it using already obtained knowledge stored in long-term memory.

A major challenge of assessing the processing capacity of visual and spatial types of information is to ensure that the VSSP is isolated so that a "pure" assessment of it occurs. Tests of pure VSSP must control for the phonological recoding process that occurs simultaneously when processing visual and spatial information (Baddeley, 2002a). After the age of 7, children frequently recode visual information into phonological form in an effort to identify and remember visual and spatial information (Miles, Morgan, Milne \& Morris, 1996).

Tests must measure VSSP capacity without allowing the information to be altered or aided by the PL components (Pickering et al., 2001). The two most widely utilized tests that measure "pure" VSSP are the Corsi block task (Milner, 1971) and the VPT 
(Della Sala et al., 1997), respectively. Both tests assess the memory sequences and capacity of "pure" VSSP without allowing the individual to recode the information into phonological form (Pickering et al.). The Corsi block-tapping task requires the individual to tap an array of blocks using the same pattern and sequence of movements as the examiner. As sequencing patterns get longer, the individual begins to forget parts of the sequence. The VPT (Della Sala et al., 1997) is similar in that the longer and more complex the visual patterns, the more likely it is that the individual will forget parts of the patterned sequence.

Cognitive experimental studies using tasks such as the Corsi block task (Milner, 1971) and the VPT (Della Sala et al., 1997) have shown that the VSSP has two subcomponents: visual and spatial (Pickering, 2001a). The visual component retains nonverbal visual information (i.e., size, color, and shape of objects). The spatial component retains dimensional information about the spatial relationships (i.e., size, distance, and color relative to the location and movement between objects). Two distinct cognitive resources process visual and spatial information separately within the same system (Bull et al., 1999; Pickering; Pickering et al., 2001).

Studies on one-, 5-, 8-, and 10-year-old children administered VSSP tasks using concurrent articulatory suppression (e.g., saying the word "table") to test if articulatory suppression impairs visual or spatial working memory function. The children completed the VSSP tasks while simultaneously performing a spatial task (i.e., tapping a wooden spoon during task performance) or a verbal task (i.e., repeating words to create articulatory suppression). Task interference was measured to determine the effect of 
articulatory suppression. Simultaneous verbal tasks did not interfere with VSSP task performance. These results support the premise that visual and spatial information is maintained by separate subcomponents within the VSSP (Pickering et al., 2001). The Episodic Buffer

The most recently proposed component to the original tripartite model of working memory is the EB (Baddeley, 2003), described as a limited-capacity component that temporarily stores information that has been coded and organized by the CE. The EB is capable of holding and maintaining this multidimensional information and stores it in long-term memory for later access. Baddeley (2003) described the EB as "a limited capacity store that binds together information to form integrated episodes" (p. 836). It can be regarded as a storage component of the $\mathrm{CE}$ that is accessed by conscious awareness (Baddeley, 2003). Baddeley (2002a) explained that the EB is evidenced by a situation in which an individual has poor short-term recall but well-presented long-term recall at the same time. Methods for studying the EB are currently undergoing development.

The Development of Working Memory

The basic structure of working memory is in place by age 6 , and almost all parts of the structure experience a gradual linear increase in capacity from preschool age, about age 5, until adolescence, about age 15 (Pickering \& Gathercole, 2001). In typical development, increases in working memory capacity are evident in all parts of the system: CE, EB, PL, and VSSP (Gathercole et al., 2004). Although the structure is in place, the capacity of working memory increases in a linear fashion (Pickering \& Gathercole), with the most significant increase occurring at age 6 because of neurological 
development, which increases the capacity of the PL to store and rehearse verbal information and to recode visual and spatial information into phonological form (Gathercole et al.). Before age 7, the frontal lobe system of the brain, the CE component, as well as the PL, is not yet fully functioning (Gathercole, 1998). Therefore, before the age of 6, VSSP is better developed than the higher cognitive functions (the CE) or the PL. Children younger than 6 are not yet able to use the phonological recoding strategy. Instead, they rely on visual and spatial information capacities to understand and interact with their world. They rely on the VSSP to recall the physical forms of objects and people (Gathercole et al., 2004). As children develop, their capacity for visual and spatial IP increases (Hitch, Woodin, \& Baker, 1989); how this capacity increases, given the lack of phonological recoding skills before age 7, remains unknown (Pickering, 2001a; Pickering et al., 2001; Pickering \& Gathercole, 2001). What is known is that IP specific to visual and spatial information substantially increases between the ages of 5 and 11 (Pickering, 2001a). The development of the VSSP has been the focus of several studies (Pickering et al., 2001, Pickering, 2001b). Researchers have hypothesized that VSSP capacity may increase because of the accumulative knowledge effect (Logie \& Pearson, 1997; Miyake et al., 2001; Pickering, 2001a) and the nonlinear structure effect (McConnell \& Quinn, 2000).

Studies that support the accumulative knowledge effect have examined the relationship between simple storage tasks and complex storage, plus processing tasks that involve both the VSSP as well as the CE. In one study, 167 college students performed a series of spatial, visual, and perceptual speed tasks to examine relationships between 
VSSP and CE functioning (Miyake et al., 2001). They utilized six spatial ability tests and two spatial visualization tests to examine the role of the $\mathrm{CE}$ while placing specific demands on the VSSP. They found that spatial visualization tasks focusing on apprehending, encoding, and mentally manipulating spatial forms had the highest CE involvement. In contrast, perceptual speed tasks focusing on the speed and efficiency of perceptual judgments had the lowest CE involvement. The researchers concluded that "spatial ability factors place differential demands on executive functioning" (p. 627). This study supports the premise that increases in visual-spatial memory development may directly relate to the development of the CE. Furthermore, individuals who demonstrate low capacities for VSSP may also demonstrate a low capacity for CE, as evidenced by a decreased performance of CE tasks (Miyake et al.).

Another hypothesis proposed to explain how VSSP capacity increases before recoding capacities are available to children under age 6 is the nonlinear structure effect. McConnell and Quinn (2000) hypothesized that the VSSP is not a single, integrated, linear system. Instead, the VSSP may be fractionated into as yet unidentified parts that develop independently and at different rates without total dependency on the process of phonologically recoding information (McConnell \& Quinn). This hypothesis continues to be investigated as studies attempt to isolate the VSSP.

After age 7, the capacity of PL increases dramatically (Gathercole et al., 2004). The PL function allows individuals to remember words and learn new words (Baddeley et al., 1998). Gathercole et al. investigated the structure and development of the working memory. They administered the nine working memory subtests included in the WMTB-C 
(Pickering \& Gathercole, 2001) to a group of 575 male and female participants between ages 5 and 15. They found that the PL is in place by age 3 but that the strategic rehearsal process needed to retain information in the PL does not emerge until about age 7.

Pickering and Gathercole (2001) confirmed that children younger than age 6 rely on VSSP to understand their world because they have not yet developed the phonological recoding strategy for visual and spatial information. Therefore, children's PL is limited and consists of a phonological store that is utilized to recode visual material such as objects and pictures into words (phonological form). At age 7, the PL capacity begins to increase and reliably occur because of the development of the phonological strategy of rehearsing auditory speech material (Gathercole et al., 2004). Children begin to covertly recode spatial, visual, and pictorial forms into nouns, names, and words; rehearse the words through auditory speech; and then store the information into phonological shortand then long-term memory (Pickering \& Gathercole). Once a recoding strategy is in place, children have the ability to rehearse and store more information into phonological form. This development marks the most significant developmental increase of working memory capacity (Gathercole, 1998; Gathercole et al., 2004). As the basic structure of the working system increases linearly, the capacity of the CE also increases.

The CE is a complex structure that coordinates the action and flow of information from the domain-specific subsystems (PL, VSSP, and EB; Mueller et al., 2003). Studies in developmental neuropsychology (Welch et al., 1991) provided evidence that the linear growth of the CE significantly increases in children at age 7 . Studies have confirmed a clear correlation between the increased capacity and function of the $\mathrm{CE}$ and the 
development of the frontal lobe system (Bull et al., 1999). Developmental working memory studies (Gathercole, 1998) have been congruent with developmental neuropsychology studies (Bull et al., 1999; Welch et al., 1991) that correlate frontal lobe development with an increase in executive functioning and an increase in working memory capacity.

Throughout adolescent development, working memory capacity continues to develop as the child develops phonological codes for visual information that result in an increased memory for visual and spatial material. By age 15, working memory performance closely approaches adult levels, with variations in capacity reflective of processing speeds (Pickering \& Gathercole, 2001). The faster the individual can process information, the less time the material has to decay in short-term memory (Towse et al., 1998). Articulatory suppression studies have found that when given the opportunity, children and adults will use phonological strategies when presented with pure VSSP tasks (Miles et al., 1996). This suggests that the VSSP is in place earlier than components of the PL and that phonological coding, such as labeling and subvocal rehearsal, may enhance VSSP capacity (Pickering, 2001b).

During preadolescent and adolescent years, the capacity for PL expands as the child develops strategies to code, comprehend, remember, and store verbal information (Gathercole et al., 2004). PL is important for many aspects of children's development and is directly related to learning new words (Conners et al., 2001), an important process in reading comprehension, communication, and socialization. 
The current understanding of the development of working memory is that children younger than age 6 may lack frontal lobe development (affecting CE) and may not have the capacity to recode spatial and visual information into phonological form (Gathercole, 1998; Gathercole et al., 2004). Therefore, the present study excluded participants who were younger than age 8 and included only children between ages 11.0 to 15.0 . This exclusion was to ensure, according to current developmental research of working memory capacity, that the participants had typical chronological frontal lobe development, the capacity to recode visual and spatial information into verbal phonological form, and had developed rehearsal and storing capacities for PL functioning (Gathercole et al.).

The Assessment of Working Memory in Children

It was not until 2001 that the WMTB-C became available to assess comprehensively the PL and VSSP capacities of children (Pickering \& Gathercole, 2001; Gathercole \& Pickering, 2001). The WMTB-C encompasses the central component of working memory and the two independent subsystems defined by the MRS model. The WMTB-C provides an assessment of short-term memory functioning in children that is directly related to the working memory capacity levels defined by the tripartite structure of the MRS model (Pickering \& Gathercole, 2001). The WMTB-C comprises nine subtests. Three subtests assess the global organization and control structure that controls the flow of information throughout the working memory (CE), four subtests assess PL, and two subtests assess the VSSP. The VPT developed by Della Sala et al. (1997) is an independent test but is normed and incorporated into the WMTB-C as a subtest for the 
VSSP. The WMTB-C was designed so that the score from the VPT (Della Sala et al.) can be integrated into the total VSSP score.

\section{Working Memory and Social Skills}

Although the Baddeley and Hitch (1974) model of working memory continues to undergo further study about how the subcomponents of working memory control, rehearse, and regulate information, the basic tripartite of the working memory model has received support in the neuropsychological, experimental, and neuroimaging science literature (Bayliss et al., 2003; Gathercole, 1998; Gathercole et al., 2004). Neurological evidence supports the Baddeley and Hitch working memory model's premise that there are individual components of working memory. Positron emission tomography (PET) studies have identified three separate areas of neural circuitry and neurological activity that correlate with the storage and rehearsal components of the PL and VSSP (ReuterLorenz et al., 2000). The PL activates the left hemisphere of the frontal lobes, the VSSP activates the right hemisphere, and the CE activates the bilateral frontal cortex (Smith \& Jonides, 1999; D’Esposito et al., 1995). The CE processes increased activity in the dorsolateral prefrontal cortex (Gathercole \& Pickerington, 2001).

These disciplines provide convergent evidence of separate functional systems underlying PL and VSSP that relate to specific right and left hemispheric functions. Studies of neurological damage have illustrated that right hemisphere damage correlates with poor visual skill performance while verbal skill performance remains constant (Farah, Hamond, Levine, \& Calvanio, 1988; Hanley, Young, \& Pearson, 1991). Empirical data from psychological and neurological perspectives have confirmed that 
phonological and visual-spatial information are separate, just as the Baddeley and Hitch (1974) tripartite model suggests. The consequence of poor VSSP for everyday social interactions is unknown. Social interactions require that the individual process spatial and visual information such as eye contact, facial expressions and idiosyncratic cues. It makes sense that working memory capacity, VSSP and CE, may be associated with that deficit. However, no studies have examined the differences between individuals with social skill deficits characteristic of AD and individuals without social deficits in terms of working memory capacity. The present study examined this relationship. The MRS model of working memory advanced initially by Baddeley and Hitch guided the theoretical basis of this project.

\section{Asperger's Disorder and Social Deficit}

$\mathrm{AD}$ is a PDD with essential features of severe and sustained impairment in social interactions; restricted, repetitive, and stereotyped patterns of behaviors and interests; and disturbances in occupational areas of functioning (Myles \& Simpson, 2002). This condition was first observed in the 1940s, but it was not included in the DSM as an independent diagnosis until 1994. Although AD is listed as an independent subcategory under PDD in the DSM-IV-TR (APA, 2000), controversy remains about whether AD is an independent category or part of a continuum along a spectrum of similar PDDs.

\section{The History of $A D$}

In 1944, Hans Asperger, a Viennese physician at Vienna University Children's Clinic, described the clinical cases of four boys who demonstrated general cognitive functioning in the normal range but also demonstrated severely impaired social 
communication skills, rigid and excessive adherence to routines, and obsessive patterns of interests (Attwood, 2004; Freeman, Caonin, \& Candela, 2002; Gutstein \& Whitney, 2002; Tani et al., 2004). Asperger was aware of similar cases described one year earlier by Leo Kanner, who identified 11 children as having autistic psychotropic disorder. He observed that these children related better to objects than to people.

Asperger acknowledged that although the boys he observed shared similar social deficits, he believed that his cases represented a distinct and independent condition (Myles \& Simpson, 2002). Asperger noticed that the boys he observed did not have delays in speech, as Kanner's cases did, but had more motor deficits than Kanner's cases. Additionally, the age of onset in Asperger's cases was later than Kanner's cases, and all of the initial cases observed by Hans Asperger were boys (Gilliam, 2001; Rinehart, Bradshaw, Brereton, \& Tonge, 2002a).

Asperger's observations remained relatively unknown until 1980, when Lorna Wing translated them into English and published a paper on 35 individuals ages 5 to 35 who displayed symptoms of the disorder described by Asperger (Myles \& Simpson, 2002). This translation sparked interest in the disorder. Eventually, the DSM-IV-TR included AD as an independent category (APA, 2000).

Diagnosis and Criteria of $A D$

$\mathrm{AD}$ is an independent diagnostic category in the DSM-IV-TR (APA, 2000) and the $10^{\text {th }}$ revision of the International Classification of Disease (ICD-10, World Health Organization [WHO], 1992). In the $D S M-I V-T R, \mathrm{AD}$ is identified as a PDD with an onset during infancy and childhood. The DSM-IV-TR describes PDDs as severe and persistent 
impairments in several areas of development. Characteristics of PDD may include deviations from typical development due to stereotyped interests, behavior, and activities; poor reciprocal social interaction skills; and/or poor communication skills (APA). The diagnostic criteria for $\mathrm{AD}$ include qualitative impairments in social interactions that include the inability to develop age-appropriate peer relationships; difficulty using multiple nonverbal behaviors such as eye contact, gestures, facial expressions, and eye gaze; lack of social or emotional reciprocity; and the inability to share spontaneously enjoyment, interests, and achievements with others (APA). Social incompetence is significant in that individuals with $\mathrm{AD}$ are unable to understand and interpret the nonverbal cues and expressions of others, despite the absence of language delays, cognitive impairments, and/or delays in self-help skills and adaptive behaviors unrelated to social interactions (APA; Barnhill, Cook, Tebbenkamp, \& Myles, 2002).

Behavioral impairments include restrictive repetitive and stereotyped patterns of behavior. Behaviors may manifest as an abnormal and intense focus with one or more restricted and stereotyped interests, an inflexible adherence to nonfunctional rituals and routines, stereotyped and repetitive motor mannerisms, and/or a persistent preoccupation with parts or objects. Qualitative and behavioral disturbances cause clinically significant impairments in social, occupational, and other areas of functioning (APA, 2000).

There is some controversy about the diagnostic criteria for AD as defined by the $D S M-I V-T R$ (APA, 2000) involving qualitative and behavioral descriptions. The diagnosis identifies criteria as qualitative impairments in communication and social skills, despite the absence of language delays. The criteria describe "no significant 
language delays" as "single words used by age 2 years, communicative phrases used by age 3 years" (APA, p. 84). However, clinicians such as speech therapists have argued that language development, as described in the DSM-IV-TR, is a description of delayed language development, not normal development, (Attwood, 2004). This confusion questions the use of "early language delay as a differential criterion for Autism and Asperger's disorder" (Eisenmajer et al., 1998, p. 532).

There is also controversy about the behavioral descriptions of $\mathrm{AD}$ in the $D S M-I V$ $T R$ (APA, 2000). Miller and Ozonoff (1997) examined the four cases that Hans Asperger observed and determined that according to the current DSM-VI-TR diagnostic criteria of $\mathrm{AD}$, the boys would have been diagnosed with autism, not AD. The present criteria of AD do not capture the original description of this disorder (Miller \& Ozonoff). Instead, the $D S M-I V-T R$ diagnostic criteria of AD are superficial guidelines and that clinical data are more specifically descriptive of AD. The differentiation of language delay, behavioral characteristics, and age of onset should be used as a guideline, not as an exclusive tool for diagnosing the disorder (Attwood, 2004).

Diagnosing AD entails the completion of a comprehensive history of past and current behaviors, language delays, and social skills development (Gilliam, 2001). There are structured assessment tools available to aid in this process. The GADS (Gilliam, 2001) was used in this study to establish inter-rater reliability and eligibility for group placement. AD affects the individual throughout the life span. Symptoms that impair typical functioning and development are influenced by one's age, peer expectations, and ability to cope and adjust to age-appropriate situations (Baron-Cohen, O'Riordan, Stone, 
Jones, \& Plaisted, 1999). Although the present study utilized the GADS to obtain an ADQ score, the participants were independently diagnosed with AD prior to the study.

To diagnose an individual, a comprehensive assessment must be completed, which entails more than a single ADQ score yielded by the GADS.

$\mathrm{AD}$ occurs more often in males than females for reasons unknown (Bauer, 1996). The ratio of boys to girls is estimated at 4.7:1 (Attwood, 2004). In 1996, Bauer estimated that $\mathrm{AD}$ occurs in 20 to 25 per 10,000 births; in 1999, estimates increased to 48 per 10,000 births (Kadesjo, Gillberg, \& Hagberg, 1999). Increased prevalence rates may be due to better recognition of symptoms (Volkmar et al., 2004) or to an actual increase in the frequency of the disorder. A total population study in 1992 of 826 7-year-old children in Karlstand, Sweden, all of whom were diagnosed with autism or AD, suggested that $1 \%$ of the general population is affected. Most researchers have agreed that $\mathrm{AD}$ is a lifelong disorder (Kadesjo et al.) and that it frequently runs in families (Howlin \& Asgharian, 1999). There appears to be no association between autistic spectrum disorders and social class (Fombonne, 2003) or environmental factors (Wing \& Potter, 2002). Howlin and Asgharian compared the diagnostic experiences of 614 parents of children with autism and 156 parents of children with $\mathrm{AD}$ and found that the average ages of diagnosis for $\mathrm{AD}$ fall between 11 and 13 .

AD versus Autism

$\mathrm{AD}$ and autism are listed separately in the $D S M-I V-T R$ as subtypes of PDD (APA, 2000). However, controversy continues about whether or not the diagnoses are two distinct conditions with separate neurobiological underpinnings or if they comprise one 
condition that exists on a symptom continuum (Miller \& Ozonoff, 2000; Myles \& Simpson, 2002; Rinehart et al., 2002b). Research has focused on distinguishing similarities and differences between the two subtypes (e.g. Gilliam, 2001; Gilotty, Kenworthy, Sirian, Black \& Wagner, 2002; Gunter et al., 2002).

The similarities between the conditions (i.e., social isolation, the lack of spontaneous social interactions, difficulty with language, lack of spontaneous play, difficulty with idiosyncratic phrases, difficulty interpreting nonverbal communication, and lack of interest in others' feelings) support the premise that AD is a mild form of autism (Miller \& Ozonoff, 2000). Conversely, motor and speech delay differences support the premise that they are two distinct conditions. Motor deficits are more common in individuals with $\mathrm{AD}$ and in general, more boys are diagnosed with $\mathrm{AD}$ than with autistic (Freeman et al., 2002; Gilliam, 2001). The symptoms of social deficits and behavioral difficulties in autism are identified at an earlier age (mean age at about 5.5 years) than in $\mathrm{AD}$ (mean age at about 11 years). Delayed speech and language skills are clinically present in autism, but not so in AD (APA, 2000; Freeman et al.; Howlin \& Asgharian, 1999).

In clinical literature, controversy remains as to whether speech and language skills develop normally with children who are diagnosed later with AD (Attwood, 2004; Eisenmajer et al., 1998). If not, verbal IP skills may be affected and may reflect deficits in the PL. This was investigated in this study as identified as Null Hypothesis 1, which stated that PL capacity of AD individuals and typically developing peers may not be significantly different. Alternative Hypothesis 1 stated that PL capacity of AD 
individuals, compared with typically developing peers, would be significantly different. Support for Null Hypothesis 1 of this current study was found in a study comparing the conversational behaviors of 19 boys with $\mathrm{AD}$ and 19 boys with conduct disorder (Adams, Green, Gilchrist \& Cox, 2002).

Each group engaged in two experimentally controlled conversations: one general conversation, and the other emotionally and socially loaded. Findings suggested that the boys with $\mathrm{AD}$ showed more pragmatic problems than the control group in both conversations, but more so in the emotionally and socially loaded conversation (Adams et al., 2002). Pragmatic problems included taking turns during the conversation, initiating conversation, spontaneity, and the number of utterances. However, no differences were found between the groups in verbal comprehension of the content or questions presented. Verbal comprehension skills may indicate that the individual is processing verbal information within the normal limits of working memory capacity (Pickering \& Gathercole, 2001). Adams et al. concluded that verbal memory and comprehension were not deficits of the boys with AD.

Some studies investigating the neurobiological differences between AD and autism have hypothesized the existence of differences between performance IQ and verbal IQ (Harvard Mental Health Letter, 1999). Gunter et al. (2002) confirmed this hypothesis and found that children with AD show higher verbal IQ than performance IQ and that children with autism show the opposite profile. This cognitive profile difference remains controversial because studies have contradicted the findings of IQ profile differences. For example, Barnhill et al. (2000) reviewed 22 studies that analyzed 
Wechsler IQ score differences of individuals with AD and found inconsistent profile patterns within the group with AD. They conducted their own study to determine if there is a cognitive profile characteristic of individuals with AD. After analyzing the Wechsler profiles of 37 children and adolescents, they found no specific cognitive profile pattern among individuals with AD or autistic. They concluded that the only distinguishable symptoms between the two groups are behavioral.

Uncertainty remains, in part, because IQ patterns remain mixed when comparing profiles of individuals within each group (David, David, \& Riley, 2003; Ghaziuddin \& Mountain-Kimchi, 2004). Inconsistent findings in cognitive profiles, especially verbal performance, of individuals with characteristics of $\mathrm{AD}$, support the need to investigate differences in IP capacity, such as those related to working memory.

\section{AD Across the Life Span}

$\mathrm{AD}$ is difficult to diagnose in children before age 3 or 4 . Although concerns presented at this age may include language delays, odd verbal responses, problems sustaining conversations, and motor clumsiness, these delays are usually not considered significant because many children catch up by age 5 (Bauer, 1996; Holaday, Moak \& Shipley, 2001). In Kindergarten, children may be viewed as somewhat odd because of obsessive areas of interest, but usually only severe problems with overly immature social skills and peer interactions merit further assessment (Bauer). Severe problems are usually obvious and include the inability to use basic gestures or display a reciprocal nature of play or an affect congruent with the social situation (Holaday et al.). 
During the early elementary age years, parents often identify social problems as being more significant than teachers do. However, as the children entered late elementary and middle school, teachers' ratings of social problems became more significant, exceeding the at-risk category. The children continued to show academic strengths but displayed weak friend-making and friend-keeping skills, evidence of a lack of social competence development (Gutstein \& Whitney, 2002). The children had difficulty learning and applying social rules, displaying obsessive areas of interest, and applying common sense to peer interactions. In these cases, a comprehensive assessment may be administered to determine if the children meet the criteria for AD (Myles \& Simpson, 2002).

During the middle school and adolescent years, children with AD deviate from typical peer interaction behaviors and have difficulty deciphering nonverbal behaviors such as tone of voice, gestures, nuances, and facial expressions. They are often seen as "weird" or "geeky" by their peers (Little, 2003). They often display minimal social interactions with peers, odd and self-focused conversations, poor eye contact; fail to understand emotional reciprocity; are unable to share enjoyment and/or spontaneous conversation with others; and have a significant lack of peer friendships (Carter et al., 2004). Social reasoning is deficit in individuals with AD, who are unable to plan, organize, shift attention, and control impulses when involved in social situations. They cannot understand and respond to emotions of anxiety, sadness, anger (Attwood, 1998); such responses require CE skills. "These emotions inhibit rational thought, a process associated with the frontal lobes" (Attwood, p. 5). 
An example of social difficulties was evident in a study that investigated the ability of preadolescents and adolescents with $\mathrm{AD}$ to identify faux pas during social interactions in comparison to typically developing, same-age peers (Baron-Cohen et al., 1999). To understand and identify faux pas, one must be able to detect "sarcasm, bluff, irony and double bluff” (Baron-Cohen et al., p. 407). The IP required to perform the complex task of detecting, recognizing, and preventing faux pas behavior during social interactions is directly related to the IP of complex tasks described in the MRS model of working memory (Baddeley, 1996, 2001). The model identifies the CE as responsible for organizing and manipulating information in the working memory (Baddeley, 2003).

Baron-Cohen et al. (1999) found that the individuals with AD in their study identified significantly fewer faux pas than the controls did. They observed that some individuals with AD could identify faux pas in the stories presented "and yet were observed to commit faux pas in their everyday behavior" (p. 415). They suggested that future research investigate methods for assessing this IP deficit of everyday behavior. This study supports the premise that working memory may play a role in social perception capacities; however, in the Baron-Cohen et al. study, specific types of IP capacities inherent in the ability to recognize faux pas was not investigated.

Everyday difficulties related to social adjustment and behavioral difficulties continue throughout high school for adolescents with AD. They are often left out, misunderstood, and teased by peer groups, where conformity is desired and tolerance for differences is low (Bauer, 1996). Bellini (2004) suggested that the pressures of the social environment cause adolescents with AD to become anxious because of their inability to 
"meet the increased interpersonal demands of adolescence" (p. 84). Many times, adolescents use masquerading to cope with the demands of peer relationships (Carrington et al., 2003). A qualitative study investigated the perceptions of friendship of teenagers with AD. Semistructured interview data suggested that adolescents with AD may define friendships and value friendships differently than professionals and/or typically developing peers. In Carrington et al.'s study, they were able to identify whom they "would not" choose as friends, but they had difficulty identifying whom they "would" choose as friends.

As adults, individuals with AD continue to demonstrate social skill deficits and a lack of social competence. The inability to establish significant social relationships means that many people with AD are unemployed, underemployed, and unable to live independently (Gutstein \& Whitney, 2002). Individuals with AD who are employed gravitate toward careers in their special areas of interest and demonstrate proficiency, but they find the emotional challenges of marriage and adult peer relationships difficult (Bauer, 1996).

Throughout the life span, children with odd social behaviors and those who lack social competence and skills miss social cues are unable to learn age-appropriate social interactions, and they continue to experience social problems (Bauer, 1996). It is possible that children who cannot interpret nonverbal social cues (Gutstein \& Whitney, 2002); decipher nonverbal behaviors such as tone of voice, gestures, nuances, and facial expressions (Little, 2003); and develop and maintain friendships (Carrington et al., 2003; 
Carter et al., 2004; Gutstein \& Whitney) lack the ability to process information related to CE.

The IP capacity related to working memory is inherent in social interactions and relationships. For example, at age 6, children rely on visual and spatial IP skills to understand and interact with their environment because of the lack of phonological recoding skills (Gathercole et al., 2004). This premise was investigated in this study as Null Hypothesis 2, which stated that the VSSP capacity of individuals with AD in comparison with their typically developing, same-age peers may not be significantly different. Alternative Hypothesis 2 stated that the VSSP capacity of individuals with AD in comparison with their typically developing, same-age peers was significantly different. Given that the alternative hypothesis was true, it may be that children who lack visual and spatial IP skills cannot recode, manipulate, and organize this information into an interpretable and integrative form related to a novel social situation. Therefore, young children with AD may lack visual or spatial cognitive IP skills related to working memory that are necessary for appropriate social interactions as they grow older. Deficits in IP skills related to working memory capacity may interfere with social competence and social skill development. Deficits in specific types of IP (PL and VSSP) as they relate to working memory were investigated in this study.

\section{Evolutionary Perspective of Social Interactions}

Weak social skills, such as problems sustaining conversations, overly immature social skills, and peer interactions, are characteristic of individuals with AD (Bauer, 1996; Holaday et al., 2001). These problems continue throughout child development and 
remain chronic problems for some individuals as adults. Social isolation is not congruent with the normal evolution of the human brain.

The social brain hypothesis (Dunbar, 2003) argued that the evolution of the brain has been influenced by humans' need to bond and interact with large social groups, which has resulted in increases in cognitive demands and in the executive brain or neocortex area (Dunbar). According to the evolutionary anthropology perspective, the social brain hypothesis is supported by evidence of increases in anatomical and functional human brain development. Therefore, not belonging to a social group is an unnatural state of being because social exchange is universal (Nettle \& Dunbar, 1997) and "human societies are characterized by high degrees of reciprocal altruism between unrelated individuals" (Cox, Sluckin, \& Steele, 1999, p. 369).

Dunbar (2004) purported that the advanced forms of social cognition shared by humans are the result of primate brain evolution and that the evolution of the human brain led to the ability to develop the capacity for theory of mind (Cox et al., 1999; Dunbar). This ability promotes relationships with others based on exchanging information, communicating deep inner feelings, and experiencing a sense of warmth toward another. Language serves the purpose of increasing the size of group interactions for an individual (Dunbar). Social altruism developed after years of small- and largegroup social interactions under certain conditions (Cox et al.; Trivers, 1971). The conditions under which individuals sustain and develop social interactions were explored in the literature. 
Cox et al. (1999) conducted a study of large-group interactions and found that cooperation between members is sustained if the individuals can store information about past play and social interactive experiences, coordinate relationships with other group members, and process information effectively (Cox et al.). The findings are congruent with those of Dunbar (1992). The capacity for memory is a prominent feature of reciprocal social behavior in terms of the ability to remember and store information about relationships with others and to "assess other's intentions using a memory function to compensate for the limited number of interactions" (Cox et al., p. 374).

As Dunbar (2003) clarified, the social brain hypothesis has established positive correlations between increases in volume of the executive brain and the human ability to behave cooperatively in a group and learn social skills. Dunbar explained that the social brain hypothesis has not tested if the cognitive powers necessary to interact socially with others can explain social intelligence. Rather, there are two different levels of social survival. She commented:

One is whether individuals use social transmissions of information to solve problems of day-to-day survival and reproduction, and the other is whether individuals are able to exploit and manipulate the mid-states of other individuals in managing the social relationships on which their day-to -day survival and reproduction depend. (p. 167)

Dunbar (2004) suggested that the social brain hypothesis does not conclude that large brain sizes gives humans the ability to interact socially. Rather, social interactions may be a "reflection of the ability to use basic executive functions in a more sophisticated way" (Dunbar, p. 167). Evolutionary anthropology has correlated social altruism and social cooperation with anatomical increases of the neocortex (Cox et al., 1999; Dunbar, 
2003); increased capacity of executive functioning; and the capacity to remember, store, and coordinate information within a social context. However, one's capacity to demonstrate social reciprocal interactions, empathy, and exchange is not fully explained by this theory or anatomical brain increases (Dunbar, 2003).

The capacity of some individuals, but not all, to utilize executive functions in cooperative social is not explained by evolutionary anthropology. Executive functions that include the ability to code, store, manipulate, and retrieve social information (Kibby et al., 2004; Miyake et al., 2001) are closely related to CE in terms of organizing materials, planning skills, initiating problem-solving methods, and sustaining attention and generalized memory. This premise was investigated in this study as Null Hypothesis 3, which stated that the CE capacity of individuals with $\mathrm{AD}$ in comparison to their typically developing, same-age peers may not be significantly different. Alternative Hypothesis 3 stated that the CE capacity of individuals with AD in comparison tot heir typically developing, same-age peers will be significantly different. Given that the alternative hypothesis was true, the CE skills needed to simultaneously process multiple types of information during social interactions may be deficit in individuals with AD.

\section{Social Deficit and Cognitive Deficit Hypotheses}

Although the central features of $\mathrm{AD}$ are difficulty understanding the rules, nuances, and reciprocal exchanges of social interactions, the nature and underlying causes of this social deficit remain unclear (Bonnet \& Gao, 1996). Despite an abundance of research to identify and understand the specific social deficits in $\mathrm{AD}$, the results have been inconclusive and conflicting because of variations in the definitions of social 
abilities and the range of how deviations from social skill norms are identified.

Conflicting results may also be the result of heterogeneity within AD subject groups because study samples may have included individuals with autism and/or individuals with AD and comorbid disorders (Baron-Cohen et al., 1999; Cohen, Schmidt-Lackner, Romanczyk, \& Sudhalter, 2003; Hadwin, Baron-Cohen, Howlin, \& Hill, 1997; Klin, 2000; Morgan et al., 2003; Rinehart et al., 2000; Ropar \& Mitchell, 2001; Sicotte \& Stemberger, 1999).

The following section reviews the literature on social and cognitive theories and integrates research identifying the neurobiological underpinnings of $\mathrm{AD}$. The final section identifies common aspects of IP deficits related to social and cognitive theories and their relationship to the function and capacity of working memory.

Theories that Support the Social Deficit Hypothesis

The premise of the social deficit hypothesis is that the underlying social skill problems of individuals with AD are caused by IP deficits in the ability to attend to social information or cues (Dawson et al., 2004) that prevent individuals from inferring the mental state of self or others in a social context with relation to beliefs, intentions, and/or thoughts (Baron-Cohen et al., 1997; Baron-Cohen, Wheelwright, Hill, Raste, \& Plumb, 2001; Jolliffe \& Baron-Cohen, 1999). The core theories most commonly found in the literature that support the hypothesis are the theory of mind (TOM) and the social attention deficit theory.

Theory of Mind 
TOM identifies the ability to think about one's own thoughts and beliefs as well as the thoughts and beliefs of others in order to understand how others may view social interactions differently than oneself (Bauminger \& Kasari, 2000; Happé \& Loth, 2002). Baron-Cohen (1995) identified it as a fundamental ability to mind read. Individuals with AD or autism share a TOM deficit that results in the inability to surmise the desires, intentions, beliefs, and mental states of the self or others (Baron-Cohen \& Hammer, 1997; Frith \& Happé, 1999).

The ability to understand mental states occurs at two levels (Lawson, 2003). The first level of TOM is to understand and infer the mental states of others while socially interacting with them. Individuals must be able to differentiate the beliefs, pretend information (jokes), real knowledge, and thoughts of the other person and of themselves. This information is often nonverbal, visual, and inferred throughout reciprocal social interactions. "Mind-blindness" includes the ability to discern if the actions of another are accidental or intentional (Attwood, 1998).

The second level of TOM is to understand how to use inferred information and representations of mental states to predict future social/interactive behaviors of self and others. Individuals with social deficits are relatively unable to understand mental states at either level, as evidenced by their inability to infer the meaning of nonverbal and verbal information during common social interactions (Bauminger \& Kasari, 2000). They are unable to think about thoughts (Attwood, 1998). TOM skills are thought to originate from an intuitive domain where one understands, empathizes, and responds to another's feelings and thoughts spontaneously in an emotionally appropriate way (Lawson, 2003). 
The TOM has gained attention and support over the past decade; however, limitations of the TOM remain in regard to social competence and skill deficits specific to AD. Findings have suggested that individuals with social skill deficits characteristic of autism and AD cannot infer other's thoughts and engage in "mind-reading" during social interactions (Baron-Cohen et al., 1997, 2001; Jolliffe \& Baron-Cohen, 1999).

However, the TOM cannot fully explain the underlying social skill deficits specific to AD. Researchers (e.g., Dyck, Ferguson, \& Shochet, 2001; Tager-Flusberg, 1999; Sicotte \& Stemberger, 1999; Corcoran \& Mercer, 1995; Peterson \& Siegal, 2002; Yirmiya, Erel, Shaked \& Solomonica-Levi, 1998) have found that TOM skill deficits also manifest in language impairment and mental retardation and are not exclusive to individuals with AD (Dawson et al., 1998; Volkmar et al., 2004).

Sicotte and Sternberger (1999) reported that the TOM cannot fully explain the underlying social deficit of AD. The researchers evaluated 14 matched pairs (one group with specific language disorder and one group with PDD-NOS) on brain functioning inference tasks and false belief tasks. The findings supported the hypothesis that individuals with PDD-NOS lack TOM skills. Results of this study indicated that $93 \%$ of the group participants with language impairments accurately predicted what another child might think during the false belief task, as compared to only $36 \%$ of the participants with PDD-NOS. Although Sicotte and Sternberger supported the TOM for individuals with PDD-NOS, they also found that individuals with a variety of PDD symptoms, not specifically AD, demonstrate poor TOM skills. 
Other studies have questioned the premise that TOM is exclusive to the population with PDD because children who are deaf, mentally retarded, or schizophrenic, demonstrate similar inabilities to engage successfully in TOM tasks (Corcoran \& Mercer, 1995; Peterson \& Siegal, 2002; Yirmiya et al., 1998). Although TOM deficits are present in individuals with PDD, TOM deficits lack specificity to autism and AD (Dyck et al., 2001; Tager-Flusberg, 1999; Volkmar et al., 2004).

The TOM is limited in its application to individuals who demonstrate symptoms of PDD because TOM skills develop after PDD symptoms emerge. Dawson et al. (1998) questioned if TOM skill deficits are specific to the social impairments of individuals with autism and PDD-NOS. They conducted retrospective studies of autistic children by watching videotapes of first-year birthday parties of children who were later diagnosed with autism. They found that the children exhibited difficulties in social skills, as evidenced by covering their ears, engaging in self-stimulating behaviors, responding less frequently to their names, attending to objects more than to people, and demonstrating a lack of attention to social interactions than their same-age peers.

Dawson et al. (1998) asked how these social behaviors directly relate to the development of TOM skills, because social impairments are obvious before TOM skills fully develop. It is not until about age 4 that children understand that others can have thoughts, beliefs, desires, and influences that are different from their own (Lawson, 2003). Dawson et al. used social orientating impairment to describe observations of oneyear-old children and ponder if deficits in TOM skills can sufficiently explain the lack of spontaneous response to social stimuli observed. 
Another limitation of the TOM in regard to $\mathrm{AD}$ is that it cannot explain functional behavioral and cognitive problems specific to individuals with AD (Klin, 2000) and their inability to generalize newly acquired TOM skills to novel, real-life situations (Dyck et al., 2001; Hadwin et al., 1997; Klin). Klin suggested that a "theory of TOM in action is required in order to bridge the gap between TOM capacity and performance" (p. 841). The gap between capacity and performance also is applied to long-term effects (Parsons \& Mitchell, 2002). They found that the efficacy of teaching individuals with AD TOM skills and social-behavior skills is situation based. However, it is generally unsuccessful in the long term because the skills demonstrated during structured training are not easily generalized (Parsons \& Mitchell).

At best, the TOM only partially explains the social deficits demonstrated by individuals with $\mathrm{AD}$ and does not account for their various behavior manifestations, such as narrow interests and preservative behavior patterns (Hadwin et al., 1997; Klin, 2000). By definition, TOM skills are complex-cognitive tasks (Little, 2003) that can result in poor social interactions when performed poorly. TOM task performance requires that individuals infer and respond to the mental state of self and others and understand how to use this information to predict future social/interactive behaviors of self and others (Corcoran et al., 1995; Dawson et al., 1998; Lawson, 2003; Peterson \& Siegal, 2002; Yirmiya et al., 1998). The complex task requirements inherent in TOM skills and the differences in levels of TOM task performance relate to the complex IP requirements of working memory and differences in working memory capacity. 
TOM skills require that individuals process several types of information simultaneously during social interactions (Little, 2003). They must utilize working memory to identify, code, organize, store, attend to, and retrieve visual, spatial, and verbal information while simultaneously rehearsing and storing information in memory (Gathercole \& Pickering, 2001) to use later when responding to social situations. Processing different types of information simultaneously to accomplish TOM skills is a dual process (Barrett et al., 2004) in which task performance is individually based. To understand this phenomenon, Barrett et al. suggested that differences in individual IP capacities be investigated in terms of working memory capacity differences to understand more clearly the dual processes that are necessary. They commented that the dual processes of working memory, such as the CE, relate to the dual processes in theories such as TOM.

The present study addressed the capacity and performance of IP skills linked to working memory capacity as defined by the MRS model. Working memory, as it relates to dual-processing tasks, such as inferring the mental state of another (TOM), is directly associated to perceiving, identifying, storing, and applying verbal, visual, and spatial information acquired in social interactions. The working memory capacity of an individual with regard to complex IP (i.e., CE) may explain one's capacity for identifying, interpreting, and remembering social cues. The capacity to process multiple types of information simultaneously may affect one's ability to infer meaning of verbal and nonverbal social situations and utilize TOM skills. 
TOM skills are possible only if the individual can process verbal and nonverbal information rapidly in real time and quickly attend to the information and "keep up with" the information during social interactions (Stone, Baron-Cohen, \& Knight, 1998). During social interactions, pertinent information needs to be stored and then later retrieved and applied to the novel situation (Klin, 2000; Parsons \& Mitchell, 2002). This process of retrieving information and/or holding information is the active working memory process (Baddeley, 2003).

\section{Social Attention Deficit Theory}

The social attention deficit theory claims that the social impairment demonstrated by individuals with $\mathrm{AD}$ is due to impairments in attending to social cues. The three main domains of social impairments of attention are social orienting, joint attention, and attention to the distress of others. The social attention deficit theory proposes that early impairments in social attention prevent children from developing the social or neurological skills for appropriate reciprocal social interactions and communicative competence (Dawson et al., 1998, 2004). Studies investigating the social attention deficit theory and individuals with forms of PDD find that social attention deficits are not exclusive to the population with $\mathrm{AD}$ and that individuals with symptoms characteristic of AD have difficulty with social orientation and joint attention.

Clark et al. (1999) focused on the overlap between symptoms of attention deficit hyperactivity disorder (ADHD) and autism. They conducted a retrospective study to evaluate parental perceptions of the level of social interactions of children initially diagnosed with ADHD. They sampled parents of 78 children diagnosed with ADHD and 
found that the children have social skill deficits similar to those in individuals with autism. Between $65 \%$ and $80 \%$ of the parents retrospectively reported symptoms of autism, "symptoms that would not be expected to occur in subjects with ADHD" alone (Clark et al., p. 54). A "lack of awareness of the feelings of others" (Clark, p. 53) was the most commonly reported symptom in the sample. Frequently reported symptoms included poor abilities to use imagination, understand nonverbal cues, and reciprocate communication.

Their (1999) findings suggested that children diagnosed with ADHD should also be assessed for levels of social competence, as evidenced by high frequencies of poor eye contact, poor personal boundaries, irrelevant remarks throughout conversations, odd forms of speech, and odd motor movements. Differences between individuals diagnosed with autistic disorder or ADHD in terms of attention to social cues revealed that the children with autism demonstrated attentional problems more selectively and seemed to be distracted by internal stimuli rather than external stimuli (Clark et al.). They also found that in general, the attentional capacity of the children improved with age, implying that developmental levels improve attentional capacity, thus improving the capacity to attend to social cues (Clark et al.).

Barnhill et al. (2000) found similar overlaps in social attentional deficits in children who manifested AD symptoms and children with other autistic-related disorders. In comparing the cognitive profiles of 37 children (mean age of 9), they found no significant differences in nonverbal reasoning, visual-motor spatial integration, and comprehension skills. Barnhill et al. reported that cognitive profiles may not reflect the 
social skill deficits evident in children who demonstrate AD or Autistic-related symptoms because they rely on rote memory skills. They explained, "Given the social impairments of individuals with Asperger syndrome, a high Comprehension subtest score would not necessarily reflect the ability to apply this rote knowledge in practical, everyday social situations" (p. 152).

Barnhill et al. (2002) suggested that future research investigate short-term auditory and sequential memory deficits that may contribute to social deficits. They concluded that assessing cognitive skills related to short-term memory may provide valuable information about the strengths and weaknesses specific to the social deficits characteristic of individuals with AD symptoms. The present study assessed short-term auditory memory and sequential memory related to PL functioning.

In addition to findings that social attention deficits are not exclusive to the population with $\mathrm{AD}$, researchers have found that joint attention and social orientation attention are more sensitive discriminators of children with AD. Dawson et al. (2004) compared the three domains of social attention with 4 clinical groups of children compared to typically developing peers: (a) $N=72$ children with autistic spectrum disorder (ASD), (b) $N=50$ children with autism, (c) $N=22$ children with PDD not otherwise specified (PDD-NOS), (d) $N=34$ children with developmental delay (DD), and (e) $N=39$ children with typical development. The groups were matched for developmental age, not chronological age, and were compared in the domains of social orientation, joint attention, and attention to the distress of others. Findings revealed that at an early developmental age (less than 9 months), social orientation (i.e., responding to 
one's name being called and looking at people) is a significant indicator for predicting later autistic symptoms than is joint attention. Dawson et al. also found that at a later developmental age (greater than 30 months), preschool-age children with autistic symptoms were not only significantly impaired with social orientation attention skills but also with joint attention (Dawson et al.).

Joint attention emerges at about 9 months of age, and the two most commonly observed joint attention behaviors are pointing to a single interest in an object and alternating eye gazes to signify that the self and another are attentive to the same event (Morgan et al., 2003). Dawson et al. (2004) found that by analyzing a larger and more carefully matched group of individuals, joint attention was the most sensitive discriminator of children with AD from DD or children who demonstrate typical development. The deficit of joint attention, in addition to deficits in attending to social orientation cues, suggested a general orientating impairment in autism (Dawson et al.). They suggested that these findings underscored the diagnostic value of assessing symptoms of joint attention deficits in addition to deficits in social orientation and that these results may help to clarify the nature of the impairment so that communicative skills are developed with specific social-attention deficits in mind.

Support for the social attention deficit hypothesis can be found in cue opposition studies, which overlap with the MRS model's definition of how IP related to working memory occurs. Cue opposition studies that compare nonverbal and verbal cues have found that people rely on nonverbal cues twice as often as verbal cues when forming judgments about an interpersonal target (Lieberman \& Rosenthal, 2001). Koning and 
MaGill-Evans (2001) commented that individuals with AD experience problematic social interactions in part because they misinterpret, neglect, or overlook nonverbal cues and have difficulty interpreting nonverbal body language, a finding congruent with the social attention deficit theory. This IP deficit relates to the VSSP, which is necessary for everyday social interactions (Koning \& MaGill-Evans). However, the processed information related to the inability to interpret nonverbal information was not investigated.

Although these studies have supported the premise that early impairments in social attention, joint attention, and social orientating relate to a deficit in the development of reciprocal social interaction skills, the social attention deficit hypothesis fails to identify the underlying causes of social skill deficits in individuals with AD. The inability to participate in experience-sharing forms of social interaction is not caused exclusively by the inability to attend to social information (Gutstein \& Whitney, 2002). They concluded that IP deficits related to short-term memory or the organization of social information and cues should be investigated to further to understand how social attention deficits relate to cognitive processes.

The social attention deficit related to social orientation and joint attention (Dawson et al., 2004) is congruent with the role of the CE, as described by the MRS model. The short-term auditory and sequencing skills suggested by Barnhill et al. (2000) directly relate to the role of the $\mathrm{CE}$, which controls the actions, scheduling, and planning of multiple cognitive activities that occur simultaneously throughout the working memory (Baddeley, 2002a, 2003; Gathercole \& Pickering, 2001). The ability to attend to 
verbal, spatial, and visual cues while waiting to respond to the spontaneous and novel social interactions at that time directly relates to working memory. The working memory stores, rehearses, and retrieves verbal and visual information that occurs during the social interaction. This study investigated the short-term memory functions of individuals with $\mathrm{AD}$, as well as the capacity to organize and sequence multiple types of information inherent in social interactions. This study investigated if the $\mathrm{CE}$ is deficit in individuals with AD compared to their typically developing, same-age peers. Poor CE capacity for working memory may explain the general deficit in experience-sharing forms of social interaction.

Theories that Support the Cognitive Deficit Hypothesis

The category of cognitive deficits proposes that the central social impairments of individuals who demonstrate symptoms of AD are caused by IP deficits related to cognitive function deficits and the inability to integrate social information into various levels of meaning (Baron-Cohen, 1989; Frith, 1989; Ozonoff, Pennington, \& Rogers, 1991). The cognitive theory deficit hypothesis suggests that specific deficits in IP and cognition disrupt the normal developmental of social skills and reciprocal communication skills, and/or impair the processing of social information. The core theories that support the cognitive deficit hypothesis are the theory of central coherence (Frith \& Happé, 1999; Morgan et al., 2003) and the right hemispheric hypothesis theory (Craig \& Baron-Cohen, 1999; Gunter et al., 2002; Rourke, 2000; Rourke et al., 2002).

\section{Theory of Central Coherence}


Frith (1989) explained central coherence as a universal feature of IP demonstrated by the ability to amalgamate and analyze details or components of an element or event and translate the information into a higher level of meaning to understand the whole or global meaning of the element or event. The theory of central coherence argues that the ability to produce a global and holistic view of an event is deficit in individuals with AD, who focus on details and local levels of the situation and do not grasp the whole picture to decipher the overall meaning (Attwood, 1998), thus demonstrating a weak central coherence (Baron-Cohen \& Hammer, 1997; Frith \& Happé, 1999; Morgan et al., 2003).

An example of how deficits in central coherence may manifest in social communication follows. If someone were to ask a typically developing adolescent or adult to describe a recently viewed movie, the individual would not expect the adolescent to respond by acting out each scene and by reciting every line from the script. One would most likely provide a summary or a holistic version of the main theme of the movie. In contrast, the individual with weak central coherence would be unable to describe the global theme of the movie (Baron-Cohen \& Hammer, 1997). Instead, this individual would provide details of a particular scene, resulting in a hyperfocus of one aspect of the movie and missing the main theme or plot.

Although processing information in this way from a social perspective causes difficulties, some argue that deficits in central coherence are a strength. Many studies have found that individuals who demonstrate a bias toward local processing, or weak central coherence, demonstrate better-than-average performance in some tasks that require the processing of parts of the whole, such as block design, embedded figures, and 
the Navon tasks (Baron-Cohen \& Hammer, 1997; Jolliffe \& Baron-Cohen, 1999; Plaisted, Swettenham \& Ress, 1999; Rinehart et al., 2000).

Studies investigating a deficit of central coherence have utilized embedded figure task experiments. Jolliffe and Baron-Cohen (1997) reported that on this test, individuals with autism and AD performed similarly by focusing on details and discovering figures within a complex global/whole shape. Individuals with autism and AD demonstrated superior ability to process details within a complex shape because the tasks themselves favored local processing skills (Plaisted et al., 1999). This same above average to superior ability to process details and the parts of the whole was found in parents of children who meet the criteria for $\mathrm{AD}$, suggesting that visual-spatial anomalies may be part of the nature of AD and autism (Baron-Cohen \& Hammer, 1997).

Jolliffe and Baron-Cohen (2001) conducted two visual experiments to assess the coherence ability of high-functioning adults with autism, adults with $\mathrm{AD}$, and normal adults as the study control. The object integration task required the participants to visually integrate a set of lines or similar lines located in an illustration of an object. The scene task required the participants to describe the scene in an illustration and quickly identify inappropriate content (Jolliffe \& Baron-Cohen). The results of this study supports Frith's (1989) theory of central coherence in that the group of individuals with autism and the group of individuals with $\mathrm{AD}$ (to a lesser degree) had difficulty integrating visual information in context of the whole picture. Jolliffe and Baron-Cohen observed that the individuals with autism and AD did not spontaneously comment about the visual incongruencies in the illustration, nor did they identify odd objects in the illustration, 
even when forced to choose objects that may have been out of place. They concluded that individuals with autistic symptoms have to put in more effort to process visual information globally, as compared to typically developing individuals.

Although studies have supported the premise that children with autism and AD more accurately find details with a complex design instead of the gestalt or global meaning of the complex shape, questions remain. Is the inability to recognize the gestalt caused by an inability to integrate details of the complex whole into a holistic picture, or it the difficulty caused by the inability to inhibit the processing of irrelevant details during task performance (Rinehart et al., 2000).

Rinehart et al. (2000) addressed this distinction and reported that the theory of central coherence does not explain the inability to recognize the gestalt. Instead, they found that attentional difficulties provide more interference to identify the global picture than did the ability to recognize the gestalt. They tested global and local IP deficits and did not find support for the theory of central coherence. They compared a group of adolescent individuals with autism to a group of adolescent individuals with AD and found that both groups could successfully process global information, a result that was inconsistent with the theory of central coherence. Instead, Rinehart et al. found that although the individuals with autism or $\mathrm{AD}$ were able to identify the global image, they had difficulty inhibiting the processing of redundant details during task performance.

The results showed that both groups lack global precedence. This deficit seems to impair individuals with autism more so than individuals with symptoms of AD. The inability to inhibit local interference when attempting to complete a task that requires the 
global processing of information may relate to a right hemispheric dysfunction (Rinehart et al., 2000).

Although studies have supported the notion that skills of central coherence play an important role in the ability to communicate and comprehend social situations (Jolliffe \& Baron-Cohen, 2001; Plaisted et al., 1999; Rinehart et al., 2000), there is no conclusive evidence about the etiology of deficits in social competence characteristic of AD. Right Hemispheric Deficit Theory and Neurological Underpinnings

Neuroimaging, neuropsychological, and lateralization studies involving individuals with autism and AD have pointed to a possible link between right hemispheric dysfunction and $\mathrm{AD}$, thus supporting the premise of the right hemispheric deficit theory hypothesis. The hypothesis suggests that in general, individuals who meet the criteria for autistic symptoms manifest characteristics congruent with left hemispheric deficits, as evidenced by early language delays and difficulties with mechanical verbal skills (Craig \& Baron-Cohen, 1999; Rourke et al., 2002). Individuals who manifest the criteria for AD have impaired right hemispheric dysfunction, as evidenced by intact mechanical language skills, but impaired abilities in visual-spatial perception, visual memory, nonverbal concept formation, and higher order reasoning abilities (Casanova, Buxhoeveden, Switala \& Emil, 2002; Gunter et al., 2002; Gutstein \& Whitney, 2002; Rourke et al., 2002).

Lateralization studies comparing right hemispheric functioning and differences between left and right hemispheres of individuals with autism or AD have generated results about possible differences in right and left hemispheric functioning. Rinehart et al. 
(2002a) suggested that in general, left hemispheric dysfunction was significantly impaired in individuals with autism when subjected to the demands of executive functioning tasks, but not in individuals with AD. They found the presence of right hemispheric dysfunction in individuals with $\mathrm{AD}$ and autism, as evidenced by visualperceptual anomalies affecting higher order executive functions. They used the term executive function to reflect "the role of the frontostriatal region in coordinating cognitive-motor output so that behavior is well timed, planned, adaptable, appropriate and relevant” (p. 322). Additionally, right hemispheric dysfunction, not left, may explain why children with AD typically perform better on verbal IQ tests than performance IQ tests (Marshall, 2002).

Neuroimaging studies and structural comparisons are generated by the notion that social skills are evolutionally founded. Because most beings live in societies, it is clear from an evolutionary view that social competence has a neurological basis and that social interaction skills are a means of developing and actualizing social competence (David et al., 2003). Iwanaga, Kawasaki, and Twuchida (2000) noted that the original symptoms of neurological deficits of individuals with AD can be observed in preschool children who have yet to develop secondary symptoms of the disorder, namely, social skills. They tested this theory and investigated preschool-age children who demonstrated original symptoms of $\mathrm{AD}$ such as rigid movements, awkward walking, and stiff body posture. They compared 231 children who met the criteria for either autism or AD. Their findings determined that sensory motor functions were lower in the group with AD than the group with autism, possibly identifying an underlying neurological impairment of movement 
that is not the result of delay, immaturity, motor skill deficits, or below average intelligence.

Neurobiological studies have investigated several brain areas thought to be responsible for social interactions. Researchers have attempted to identify differences in the brains of children with AD and typically developing children by investigating brain size, brain volume, and lateralization functions (Filipek et al., 1999). They found that the heads of children with AD grow more rapidly after birth than those of typically developing children. Brain volume and head circumferences were larger in some children with $\mathrm{AD}$, but the researchers explained that this abnormality is not consistent. Neurobiological studies have not identified a causal relationship between brain structures and the symptoms of $\mathrm{AD}$, and they have offered no information about the neurological underpinnings of AD (Casanova et al., 2002). Researchers have agreed that problems such as the inability to plan and/or make accommodations to novel situations relate to impaired structures and functions of the brain (Gunter et al., 2002).

Structural neurological impairments are linked to right hemispheric brain functioning. A postmortem neurobiological study of the brains from two adults diagnosed with $\mathrm{AD}$ found no degenerative changes within the brains, but they did find minicolumnar abnormalities (i.e., smaller than normal) in the temporoparietal area of the right hemisphere (Casanova et al., 2002). The researchers suggested that this abnormality could relate to a receptive field abnormality, but they added that this finding is inconclusive. More studies with larger comparison capabilities of subjects, such as those 
identified with Rett syndrome or fragile X, need to be undertaken to identify specific temporoparietal lobe differences (Casanova et al.).

Neurological underpinnings may account for TOM deficits thought to be evident in individuals who meet the criteria for AD. Visual-perceptual IP difficulties may relate to the inability to develop a "theory of mind" commonly demonstrated by individuals with AD (Holaday et al., 2001), suggesting that the neuropsychological problems and neurological underpinnings may cause a failure to develop and ability to think about another's thoughts, beliefs, and perceptions of a social situation. Instead, individuals with $\mathrm{AD}$ focus on their own thoughts, display odd thoughts and behaviors, and seem to have no idea how to cooperate in team sports and social situations (Holaday et al.). Most studies have investigated the secondary symptoms (identified by Iwanaga et al., 2000), characteristic of and most commonly demonstrated by individuals with AD in social interactions.

Neuropsychological studies comparing individuals meeting the criteria for AD to individuals meeting the criteria for nonverbal learning disabilities have generated a theoretical framework for many symptoms of AD. Gunter et al. (2002) compared 8 children (mean age of 16.25) to individuals with AD and 8 normal controls to assess similarities between Rourke's (1989) nonverbal learning disability syndrome (NLD) model and social, academic and neurological patterns in individuals with AD. Rourke's model proposed that individuals with NLD demonstrate right hemispheric deficits due to damaged commissural fibers and white matter tracks. Rourke (2000) argued that if the commissural fibers that connect the two hemispheres are damaged, the right hemisphere 
becomes understimulated and undercommunicated with, resulting in damage to white matter tracks and a decrease of right hemispheric functioning. Because the right hemisphere has more white matter and commissural fibers than the left, damage to this hemisphere results in NLD deficits (Rourke, 1989; Rourke et al., 2002).

Rourke's (1989) model identified NLD as a primary deficit in tactile and visual perception that caused secondary deficits in tactile and visual attention and exploratory behavior, which led to tertiary deficits in the pragmatics of language (Rourke, 2000). These factors led to adaptive deficits in the areas of novel situations, emotional stability, and social competence (Greenham, 1999; Rourke et al., 2002).

Gunther et al. (2002) explored Rourke's hypothesis that individuals with NLD and individuals with $\mathrm{AD}$ have common patterns and deficits associated with deficits of right hemispheric functioning. After administering a battery of 11 neuropsychological tests, the researchers confirmed the hypothesis that individuals with AD have impaired right hemispheric functioning. The neuropsychological profiles of $\mathrm{AD}$ were similar to those of NLD individuals concerning impairments with face recognition tests, impaired social judgment; lower performance intelligence quotient (PIQ) compared with verbal intelligence quotient (VIQ), impaired right hemisphere language subtest for humor, and impaired emotion recognition for anger and disgust (Gunter et al.). They states that the Rourke (1989) NLD model of right hemispheric damage is a theoretical framework for many, but not all, symptoms of AD. The model does not explain all symptoms manifested by AD individuals, such as the inability to infer the intentions of others during social interactions. 
Although conclusive evidence about the neurological underpinnings of AD have not been established, it is suggested that right hemispheric functioning deficits and visual IP contribute to the poor IP skills that may impair social interactions (Casanova et al., 2002; Gunter et al., 2002; Gutstein \& Whitney, 2002). Social skill deficits reflective of poor IP skills may relate to the inability to identify, understand, and respond to nonverbal cues in a social situation (Koning \& MaGill-Evans, 2001).

Although individuals who demonstrate impairments in conversing with others, reading nonverbal social cues, and maintaining social relationships fail to compensate for these deficits, they are aware that they are socially isolated from peers (Rourke et al., 2002). The current study investigated the contention that the poor IP of visual and/or spatial cues inherent in social interactions may contribute to the inability to compensate for this deficit. This investigation may lead to positive social change for individuals who need to develop specific IP skills that will allow them to participate socially and experience less isolation from others.

\section{Linking Core Hypotheses to Working Memory}

The social attention and cognitive deficit hypotheses are similar in their exploration of IP deficits that individuals with $\mathrm{AD}$ have when processing complex information inherent in social interactions. They both conceptualize IP as a skill required for the processing of multiple levels and types of information. However, the cognitive and social theories do not specifically isolate IP skills into separate information types (i.e., PL, VSSP, and CE). Instead, the types of information processed simultaneously during a social task are grouped together during CE skills required in TOM (Barrett et al., 
2004); social attention (Dawson et al., 2004); central coherence (Frith \& Happé, 1999; Morgan et al., 2003); and right hemispheric functioning (Craig \& Baron-Cohen, 1999; Gunter et al., 2002). IP skills required for TOM tasks require that the individual engage in dual task performance (Barrett et al., 2004) and simultaneously process PL and VSSP to perceive, understand, and infer the intentions of others during social interactions (Baddeley, 2001; Klin, 2000; Parsons \& Mitchell, 2002). Likewise, tasks of social attention, central coherence, and right hemispheric functioning require the simultaneous processing of multiple types of information so that the individual can reciprocate social exchange. The CE skills are necessary for one to manipulate, attend to, and distinguish between whole/part relationships and relevant/irrelevant cues during novel social situations.

Although the social attention and cognitive deficit hypotheses partially explain how social and cognitive functions relate to deficits in social competence and reasoning specific to AD (Volkmar et al., 2004), CE skills in relation to these tasks have not been investigated. The automatic processing of information required to engage in tasks identified by both theories requires working memory skills. Therefore, this study supported both hypotheses in that difficulty with tasks in TOM, central coherence, social attention, and right hemispheric functioning is evidence of a deficit in IP related to a lower capacity of the CE.

The function of the CE is to perform complex working memory tasks and to control the global organization and flow of information throughout the working memory. IP skills related to working memory are required for social task performance identified by 
the theory of mind, central coherence, social attention, and right hemispheric functioning theories. It makes cognitive sense that levels of working memory capacity directly affect one's ability to perform these tasks. This present study investigated the question if IP skills related to working memory are deficit in individuals with AD in comparison to their typically developing, same-age peers.

\section{Theoretical Constructs}

RS Model of Working Memory: The RS model of working memory contends that the components of working memory share one pool of resources and that working memory capacity competes for these resources that enable the working memory to process and store information (Duff \& Logie, 2001).

MRS Model of Working Memory: The MRS model of working memory does not contend that each component of working memory shares a limited pool of resources. Instead, this model identifies two highly specialized, independent, and domain-specific subsystems supported by the CE, which is a shared central system that is domain general (Bayliss et al., 2003; Gathercole, 1998).

Social Deficit Hypothesis: This hypothesis purports that the social skill problems of individuals with $\mathrm{AD}$ are the result of IP deficits in attending to social information/cues, understanding social nuances, and inferring the mental state of self or another within a social context (Dawson et al., 1998; Lawson, 2003).

Cognitive Deficit Hypothesis: This hypothesis purports that the social skill problems of individuals with $\mathrm{AD}$ are the result of IP deficits related cognitive functioning that prevent the individuals from integrating social information into various levels of 
meaning (Baron-Cohen \& Hammer, 1997; Jolliffe \& Baron-Cohen, 1999; Morgan et al., 2003).

Theory of Mind (TOM): The TOM purports that the core social-cognitive impairment of individuals who demonstrate social deficits is the inability to infer the mental state of self and others (Baron-Cohen et al., 1997).

Social Attention Deficit Theory: The social attention deficit theory purports that individuals are unable to attend to social cues in terms of social orientation, joint attention, and attention to the distress of others (Dawson et al., 2004).

Theory of Central Coherence: The theory of central coherence purports that individuals who manifest social skill deficits are unable to translate social information into a higher level of meaning, such as the "big" picture or global meaning of the event or task (Morgan et al., 2003).

Right Hemispheric Hypothesis Theory: The right hemispheric deficit theory purports that impairments in right brain functioning are evident in visual-spatial perception, visual memory, nonverbal concept formation, and higher order reasoning abilities (Craig \& Baron-Cohen, 1999; Rourke et al., 2002). These impairments preclude the development of social skills related to emotional stability, social competence, and adaptive and novel social skills common in individuals with AD (Greenham, 1999).

\section{Current Study}

Little is known about the specific IP difficulties of individuals with AD related to the PL, VSSP, and CE capacities of working memory. This area of study may have been neglected in the past because of the unavailability of a comprehensive, reliable, and valid 
instrument to assess the working memory capacity of children. The first test to provide a theoretically sound working memory profile for children was the Working Memory Test Battery for Children (WMTB-C; Pickering \& Gathercole, 2001). The WMTB-C was utilized in this study.

It was the researcher's hypothesis that significant indicators in working memory capacity identify typically developing, same-age children from children with AD. If IP deficits in working memory capacity were better understood, remedial efforts could assist individuals with $\mathrm{AD}$ to increase their ability to identify, remember, interpret, and manipulate nonverbal and verbal cues related to social interactions. Individuals with AD who develop their working memory skills may be better able to process verbal, visual, and spatial information simultaneously, thereby improving their ability to infer the intensions of others during social interactions, a deficit not explained by current models. The present study examined this gap in the literature by comparing possible differences in the IP skills in the working memory capacity of individuals with AD to those of their typically developing, same-age peers.

The etiology, neurological underpinnings, and interventions for individuals with $\mathrm{AD}$ need to be investigated in more depth. The researcher suggests that future research should focus on the working memory capacity of individuals who demonstrate low social competence, an IP deficit that is characteristic of individuals with AD. If IP deficits in working memory were better understood, specific working memory skills could be targeted for interventions that would increase working memory capacity and improve one's ability to engage in $\mathrm{CE}$ tasks inherent in social relationships. If social functioning 
were increase, social change would occur for individuals who are painfully aware of their isolation and their inability to connect socially to others.

\section{Summary}

This chapter presented an overview of the history and development of memory theory and the RS and MRS working memory models. Complex span tasks were discussed in terms of how they have contributed to the development of the working memory model and how working memory relates to the complex tasks inherent in social interactions. Social interactions and social skill deficits were discussed within the context of the criteria for $\mathrm{AD}$. AD was discussed in terms of the essential feature of low social skills/competence, assessment, and the two major hypotheses most commonly employed to understand what causes underlying social deficits: social deficit hypothesis and the cognitive deficit hypothesis.

Common theories that support the two major hypotheses are the theory of mind, social attention deficit theory, theory of central coherence, and the right hemispheric hypothesis theory. Theories were discussed in terms of how they explain the etiology of social skill deficits, their limitations, and relationship to the concept of working memory. The chapter concluded with a discussion of how IP deficits discussed in the social and cognitive deficit hypotheses relate to the working memory research of the current study. Chapter 3 explains the design of the study, sample and population, instrumentation, data collection, and analysis procedures. 


\title{
CHAPTER 3: METHODOLOGY
}

\author{
Introduction
}

This chapter explains the research design, sample and population, instrumentation, data collection, and analysis procedures. A survey design was used to assess the working memory capacity of 30 males ages 11 to 15 with Asperger's Disorder (AD), as compared with 30 typically developing, same-age peers. The Working Memory Test Battery for Children (WMTB-C; Pickering \& Gathercole, 2001) assessed the working memory capacity of the participants.

\section{Research Design}

This quantitative study was undertaken to determine if differences between two groups of boys ages 11 to 15 (those diagnosed with AD and their typically developing, same-age peers without AD) exist in their PL, VSSP, and CE working memory capacity. This comparison ascertained if there is a relationship between working memory capacity and level of social competence. Individuals who met the criteria for either the AD group or the typical socially competent group were not randomly assigned to either condition. Instead, they were "assigned to a particular condition on the basis of some inherent characteristic" (Heiman, 2002, p. 501). In the present study, the IV was the level of social competence; the DVs, which were yielded by the WMTB-C (Pickering \& Gathercole, 2001), were the three measures of working memory capacity (verbal [PL], visual-spatial [VSSP], and complex [CE]). This study examined a between-group comparison using between-group tests to explore whether a relationship exists among the three DVs of working memory capacity. 
Description of Rater Training

Ten raters who were familiar with Gilliam's Asperger's Assessment Tool (GADS; Gilliam, 2001) and the symptoms of AD participated in two review/training sessions with this researcher to qualify as research assistants in this study. The first training session involved an orientation to the study, a review of AD symptoms, and a review of the GADS tool. The second training session involved administration of the GADS instrument while each rater observed preselected sections of a recording used to establish inter-rater reliability (Attwood, 2004). Following the session, the volunteers who had consented to participate in the study were assessed by three raters (20-30 minutes per rater) to determine if they met criteria for placement in either the AD group (A) or the typical social development group (T). All raters and research assistants signed the Research Assistant Confidentiality Agreement (see Appendix A).

\section{Setting and Sample}

The sample included 60 boys ranging from ages 11 to 15 recruited from two public school systems in Cuyahoga County, Ohio. One school is a public charter therapeutic school specializing in the education of students with special needs, primarily ADHD and AD. The other school is a traditional learning public school.

Thirty boys previously diagnosed with $\mathrm{AD}$ prior to the start of the study were placed in the $\mathrm{AD}$ group (A), and 30 boys not diagnosed with $\mathrm{AD}$ or other disorders were placed in the typical social competence group (T). The WMTB-C (Pickering \& Gathercole, 2001) yielded three working memory scores for each of the 60 participants. This was considered an adequate sample size, according to common guidelines that 
recommend that $N$ be at least 10 times the number of variables (Nunnally, 1978). According to Hair, Anderson, Tatham, and Black (1998), "The sample size must exceed specific thresholds in a cell (group) of the analysis a recommended minimum cell size is 20 observations" (p. 342). Therefore, the sample size for the present study exceeded the number of participants needed to assume a medium affect size for the three DVs (Hair et al.).

Only males comprised the study sample because AD occurs 4.7 times more often in boys than girls (Attwood, 2004). Permission to study this population was obtained from Walden University's IRB and the respective schools. The APA's (2001) ethical principles in the conduct of research on human participants (Pope \& Vasquez, 1998) were followed.

\section{Research Procedures}

After permission was granted, a notice describing the study was distributed at each school district to recruit participants (see Appendix B). The volunteers and their parents/guardians met with this researcher and/or trained research assistants to discuss the purpose of the study and to give their informed consent. The six tasks required to participate in the study were reviewed:

1. The researcher and/or a trained research assistant met with the volunteer participant and his parent/guardian to explain the purpose of the study and the requirements of each volunteer participant. Individuals who were willing to participate signed the consent forms (see Appendices C and D) and gave themselves a code number used for identification purposes throughout the data collection and analysis process. The 
code was used to maintain confidentiality and decrease researcher bias. Any questions from the prospective participants were encouraged and answered. At the end of the study, the code was available to the researcher.

2. Demographic and descriptive information (see Appendix E) was collected from each participant and parent/guardian. This process took approximately 5 minutes to complete. If any participant responded affirmatively to a previous diagnosis of Schizophrenia, Substance Dependence, Deafness, Blindness, or the inability to articulate speech, he was excluded from the study. All participants had to speak and read English and have a Full Scale IQ between 85 and 115 .

3. All participants were administered the Wechsler Intelligence Scale for ChildrenIV (WISC-IV; Wechsler, 2003) if a Full Scale IQ had not been obtained within the past 12 months. This assessment took approximately 80 minutes to complete.

4. A trained rater utilized the GADS (Gilliam, 2001) to assess each participant for a 20- to 30-minute observation. The GADS yielded an AD Quotient (ADQ) score that revealed if the participants met the criteria for placement in Group A or Group T. An ADQ score greater than 95 (low social competence) or less than 60 (typical social competence) was the criteria score set for eligibility in either group. The GADS cut-off score was for group identification purposes only and did not provide evidence of a diagnosis. The boys who were eligible to be in the AD group had received a comprehensive evaluation and diagnosis prior to and unrelated to their participation in this study. 
5. The researcher reviewed the demographic information, WISC-IV scores, and GADS assessment scores. If a volunteer did not meet the criteria for inclusion in either group, he was removed from the study.

6. The researcher administered the 9 subtests of the WMTB-C (Pickering \& Gathercole, 2001) and the short-term visual recall subtest referred to as the VPT (Della Sala et al., 1997) privately to each participant who met the criteria. Scoring for the WMTB-C was normed to include the VPT (Della Sala et al.). The testing session did not exceed 100 minutes per participant.

The researcher was blind to the specific GADS ADQ scores of each participant until after the administration of the WMTB-C. However, the researcher was not blind to the assignment of each participant to his respective group placement. Given the nature of $\mathrm{AD}$, it was nearly impossible for the researcher not to notice the lack of social competence associated with the boys in Group A.

Participants who met the criteria for inclusion completed the WMTB-C involving the 10 subtests ( 9 from the WMTB-C and the VPT) and received a form denoting their completion of the study. Collected data remained confidential and were not distributed to teachers or other school personnel. All completed paper protocols and interview data were placed in a separate yellow pocket folder and stored in a locked file cabinet. The data will be kept for 5 years in the researcher's personal office, after which time they will be shredded and discarded. 
Instrumentation and Materials

The Gilliam Asperger's Disorder Scale (GADS)

The GADS (Gilliam, 2001) is a standardized instrument for measuring symptoms and manifestations of low social competence characteristic of AD. The GADS provides norm-referenced information about levels of social competence. It can be used with individuals who have already been diagnosed with $\mathrm{AD}$ or those who may have AD. Items on the GADS are based on the definitions of AD adopted by the DSM-IV-TR (APA, 2000) and the ICD-10 (WHO, 1992).

The GADS was standardized on a sample of 371 individuals with AD from the United States, Canada, Great Britain, Mexico, Australia, and other countries (Gilliam, 2001). Statistically, the GADS is constructed such that the individual obtains an ADQ based upon a mean of 100 and a standard deviation of 15 . Because the GADS was normed on children, adolescents, and young adults with $\mathrm{AD}$, the prototypical individual who has low social competence, characteristic of $\mathrm{AD}$, can be viewed as having an ADQ of 100 . The ADQ was derived by combining the standardized scores obtained on four subscales: Social Interaction, Restricted Patterns of Behavior, Cognitive Patterns, and Pragmatic Skills. The statistical construction used to create the standardization for these four subscales was based upon a mean of 10 and a standard deviation of 3 . Each subscale has between 6 and 10 items that are rated on a Likert scale by a trained rater as one of the following responses: 0 (never observed), 1 (seldom observed), 2 (sometimes observed), and 3 (frequently observed). 
Reliability of the GADS in terms of internal consistency of the four subscales was determined using Cronbach's alpha. Alpha coefficients obtained for the subsections of the GADS were as follows: .88 for Social Interaction, .81 for Restricted Patterns of Behavior, .86 for Cognitive Patterns, and .84 for Pragmatic Skills. An alpha coefficient obtained for the ADQ was .95. Reliability for the GADS is "strong and indicates that the items within the subscales are very consistent in the measurement of characteristic behaviors of persons with Asperger's Disorder" (Gilliam, 2001, p. 8) and other serious behavioral disorders that manifest in low social competence.

The validity of the GADS has yielded consistent and discriminative subtest items. Concurrent, criterion-related validity studies have found that the scores on the GADS can be used to identify individuals with low social competence levels (Gilliam, 2001). The Wechsler Intelligence Test for Children-IV (WISC-IV)

The WISC-IV (Wechsler, 2003) is a measurement of general intelligence. It is an individually administered clinical instrument for assessing the cognitive ability of children ages 6.0 to 16.11 . The WISC-VI provides subtest and composite scores that represent intellectual functioning in specific cognitive domains, as well as a composite score that represents general intellectual ability (Full Scale IQ). The WISC-IV includes 15 subtests, 10 of which can be used to obtain a Full Scale IQ of the individual. The 10 subtests that were used in this study included Block Design, Similarities, Digit Span, Picture Concepts, Coding, Vocabulary, Letter-Number Sequencing, Matrix Reasoning, Comprehension, Symbol Search, and Picture Completion. Full Scale IQ scores were used 
for each volunteer to determine group eligibility. Individuals who obtained Full Scale IQ scores within the range of 85 to 115 were considered eligible to participate in this study.

The reliability of the WISC-IV (Wechsler, 2003) for the overall standardization sample subtests ranged from .79 (Symbol Search) to .90 (Letter-Number Sequencing). All remaining reliability coefficients were good, ranging from .80 (Word Reasoning) to .89 (Vocabulary and Matrix Reasoning). The majority of the subtests included in the WISC-IV have strong theoretical and empirical evidence of validity based on the response process (Wechsler). The validity of the WISC-IV was established through intercorrelation studies that demonstrated that the categorical definitions of the subtests included in the WISC-IV (Verbal Comprehension, Perceptual Reasoning, Working Memory, and Processing Speed) highly correlate with each other and load on the Full Scale, a general measure of intelligence. Validity of the WISC-IV has been determined by the relationship to other measures, such as the WISC-III, WIPPSI- III, WIAT-II, Children's Memory Scale (CMS), Gifted Rating Scale (GRS), and the Adaptive Behavior Assessment System. The WISC-III VIQ and the WISC-IV VIQ correlate at .87, and the PIQ for both tests is .74. The Full Scale IQ between the WISC-IV and the WISC-III is $r=$ .89 (Wechsler).

\section{The Working Memory Test Battery for Children (WMTB-C)}

The WMTB-C provides a balanced and theoretically coherent assessment of the three working memory functions: the PL, the VSSP, and the CE (Gathercole \& Pickering, 2001). The WMTB-C includes 9 subtests. Verbal short-term memory, or the PL, is measured by 4 subtests: Digit Recall, Word Listing Matching, Nonlist Recall, and Word 
List Recall. Pickering and Gathercole (2001) explained that the Nonlist Recall subtest is the most taxing on the PL system because it is the most unfamiliar to the child, as compared to digits, which are learned early in life. Because digits are familiar to most children, "the child is likely to have knowledge of the visual and semantic representations (i.e. the meaning) of each digit" (Pickering \& Gathercole, p. 80). Some children are able to represent familiar items in semantic or visual form because of limitations in their phonological skills. The compensatory strategies may allow the child to score well on PL tasks (Gathercole \& Pickering). However, these compensatory skills cannot be utilized with the Nonlist Word List Matching subtest that asks the child to recall nonsense words immediately. The Word List Matching task requires less spoken output skills than the three other PL tasks. Therefore, a child who has difficulty with the output of spoken information may perform poorly on the tasks of Digit Recall and Word and Nonword List Recall but better on Word List Matching. Hence, children with relatively good phonological working memory skills may score more poorly than their actual skill level due to difficulties with the verbal output of information (Gathercole \& Pickering).

Visual and spatial short-term memory, the VSSP, is measured by the subtests of Maze Memory Task and Block Recall. Block Recall is a spatial working memory task, whereas Maze Memory is a task that incorporates both visual and spatial information simultaneously (Gathercole \& Pickering, 2001). Gathercole and Pickering suggested that when administering the WMTB-C to obtain a comprehensive evaluation of VSSP, the individual should also be administered the VPT, developed by Della Sala (1997) and published by the Thames Valley Test Company. They commented that this test may 
highlight differences in performance of VSSP skills and provide a more detailed description of working memory skills in the area of visual and spatial information. The VPT (Della Sala et al.) was administered in the present study. This test is described in the next section of this chapter.

The CE is measured by the WMTB-C subtests of Listening Recall, Counting Recall, and Backward Digit Recall that assess the global organization and control structure that controls the flow of information throughout the working memory. Gathercole and Pickering (2001) explained that the Listening Recall task requires that the individual manipulate and retain verbal information; however, some children may represent the information in semantic or visual form. Both the Counting Recall and the Digit Recall tasks require that the individual manipulate and maintain numbers. "Evidence suggests that children who show expertise with numbers perform better on these tasks than children who have less proficiency with numbers" (Gathercole \& Pickering, p. 82). The three subtests are strongly verbal in nature. Pickering and Gathercole (2001) noted that tests that isolate the nonverbal capacity of high-level working memory (the CE functioning) have not yet been developed. Therefore, one limitation of the WMTB-C is that it does not measure the PL capacity of CE functioning. The WMTB-C is designed so that the VPT can be integrated as a single subtest into the total VSSP score (Della Sala et al., 1997). The reliability of the final version of subtests utilized with the WMTB-C was measured with 99 children between the ages of 5.4 and 11.6. Test-retest reliability on each subtest ranged from .42 to .83 . Inter-rater reliability was $.86-.90$. Construct validity is greater for the PL and the CE than for the 
VSSP; however, construct validity of the VSSP increased with the addition of the VPT (Della Sala et al.; Pickering \& Gathercole, 2001). The VPT is a test of visual short-term memory in which the individual is asked to remember visual patterns that increase in complexity as the test continues. This test was integrated as a subtest into the WMTB-C and was found to be high on the "Goodness of Fit Index (.95)" (Pickering \& Gathercole, p. 22).

The Visual Patterns Test (VPT)

The VPT (Della Sala et al., 1997) is an independent assessment of short-term visual memory, developed 4 years before the WMTB-C (Pickering \& Gathercole, 2001). Scores from this assessment that are utilized as a subtest of the WMTB-C were normed with children ages 5.5 to 15.7 to establish normative data for the WMTB-C. The VPT is a paper-pencil test. The individual is presented with blank grid paper and a checkerboard of patterns illustrated on a series of $3 \times 5$ cards. The smallest grid is a $2 \times 2$ matrix, and the complexity of the grids increases throughout the ranges of a $5 \times 6$ matrix pattern. The individual views the pattern for about 3 seconds before it is removed. The individual must then use the pencil to reproduce the pattern on the blank grid, which corresponds exactly to the dimensions on the pattern cards. The VPT is similar to other standard shortterm memory tests in that the longer and more complex the visual patterns, the more likely the individual will forget parts of the patterned sequence.

Test-retest reliability was .81 in a sample of 194 healthy British individuals ages 13 to 92 years and .88 in a sample of 76 healthy Italian individuals ages 15 to 86 . Validity of the VPT (Della Sala et al., 1997) was established by poor correlation (.060) with the 
Corsi blocks with 179 healthy British participants. Poor correlation was also evident in tests given to compare normal and brain-damaged individuals. Both groups were given forms A and B of the VPT, as compared with the Corsi block test, and the results were found to be .37 and .42 , respectively (Della Sala et al.). Poor correlation statistics showed that the VPT measures visual short-term memory and spatial short-term memory (Della Sala et al.).

\section{Diagnosis and Assessment of Asperger's Disorder}

The DVD entitled, “Asperger's Diagnostic Assessment," is a 3-hour recording that includes interviews of children and parents (Attwood, 2004). The tape was used in this study to educate and orientate the raters about AD and to establish inter-rater reliability using the GADS (Gilliam, 2001). The main subject was an 11-year-old boy who demonstrated low social competence and low social skills, characteristic of AD. Attwood, a world-renowned expert in the field of AD, interviewed the boy and his parents; after the interview, he explained the characteristics of AD. The recording illustrated social conversational skills such as eye contact, speech, nonverbal and verbal language characteristics, behavioral mannerisms, gestures, posture and the like.

\section{Administration}

The GADS (Gilliam, 2001) tool was utilized by the 10 trained raters selected to participate in this study to observe the participants for 20 minutes each. The raters completed the GADS form, and data from this form remained confidential. The ADQ was calculated by this researcher. Volunteers who obtained ADQs of 95 and above or 60 and below were eligible to participate in this study, provided that they also met other 
exclusion criteria, which were mentioned previously. The researcher and/or a certified school psychologist administered the WMTB-C and the WISC-IV. Both individuals are formally trained test administrators who are licensed professional clinical counselors (LPCC) in Ohio.

Test administrations occurred in a quiet testing office at each school. All tests were administered individually. Directions for each instrument were explained to each participant to ensure a complete understanding of the process. Each participant was given the subtests in the same order for consistency and was allowed as much time as needed to complete each one according to standard procedures, as defined by the test manual, before moving on to another one. At the end of the testing process, each participant was given the opportunity to ask any additional questions and to debrief from the experience.

\section{Data Analysis}

Thirty male subjects (aged 11.0-15.5) were included in each subject group. The IV was the level of social competence determined by the ADQ on the GADS assessment. An ADQ score of 95 and above met the criteria for placement in Group A, and an ADQ score of 60 or below met the criteria for placement in Group T. Volunteers who scored a final ADQ greater than 49 and less than 96 were excluded from the study. The DVs were PL, VSSP, and CE scores, determined by the three working memory scores yielded by the WMTB-C (Pickering \& Gathercole, 2001).

Ten raters acted as research assistants to carry out various tasks during the study, including assessing the volunteers by using the GADS (Gilliam, 2001) to determine eligibility for placement in Group A or Group T. Inter-rater reliability was established by 
the raters, who assessed selected parts of a DVD recording of children who manifest symptoms of low social competence, characteristic of AD. The GADS (Gilliam) tool was used to establish rater-scores for the children illustrated on the tape. Lin's Concordance Coefficient (rl) was used to measure inter-rater reliability. It measures the level of covariation between raters for interval or ratio data (Likert scales meet this criterion). The GADS assessment tool is based on a Likert scale to score each item, so it is appropriate to establish inter-rater reliability using the Lin's Concordance. This particular statistic is superior to Pearson's Correlation Coefficient (r) in that it takes into account systematically higher codes of one rater versus another (Neuendorf, 2002).

The DVs were the subtest scores on the WMTB-C that yielded PL, VSSP, and CE scores. To distinguish between the two groups, differences in the mean scores from each subtest would have to reach a significant level. The most appropriate statistical test for this design were three one-way analyses of variance (ANOVAs) with directional hypotheses. As mentioned in chapter 2, the literature suggests that given their IP deficits, AD individuals tended to have lower scores on each of the tests of working memory. Significant results indicated if differences existed between the two samples formed by the IV on each of the DVs. A set of ANOVAs were computed, resulting in three F-ratios (Heiman, 2002) to determine if significant differences existed among PL, VSSP, and CE working memory capacity.

For the use of this technique to be valid, the values of the DVs must be normally distributed, and the participants obtained as random samples from their respective populations. A study using a minimum of 30 participants per variable, per group, 
provided enough degrees of freedom to look for statistical significance to ensure normal distribution. Therefore, using a sample size of 30 participants per group and an alpha level of .05 would yield a normal distribution and believable relationship within the respective populations (Hair et al., 1998; Heiman, 2002).

The sample's mean differences were computed to summarize the sample's relationships for each variable. The differences in scores were compared between conditions relative to the error variance within conditions. Because no prior studies have investigated differences in specific types of working memory capacity between individuals with AD and typically developing, same-age peers, there is no direct evidence to assume the effect of the differences between the groups on test scores. "This suggests that the researcher, if anticipating the effects to be small, must design the study with much larger sample sizes and/or less restrictive alpha levels (.05 - .10)" (Hair et al., 1998, p. 12). Therefore, the present study utilized the standard assumption when considering the interrelationship of sample size, effect size, and alpha level. The standard assumption was satisfied with 30 participants per group, or 10 participants per DV, and a less restrictive alpha level of .05 (Jaccard \& Becker, 1997).

A MANOVA, which is commonly used to measure two or more DVs (Heiman, 2002), was not used in this study to look for a statistically significant difference with regard to all three DVs. This analysis would have compared the overall working memory capacity for $\mathrm{AD}$ individuals to that of their typically developing, same-age peers. The advantage of using a MANOVA would have been that it would have eliminated the 
additive element of running several statistical tests within the same sample commonly referred to as alpha build up or alpha-pyramiding (Hair et al., 1998).

The disadvantage was that a MANOVA would have tested the sum of all three DVs at once, thereby identifying overall working memory capacity as a single construct in which we understand the relationship among the three subtest categories. The literature acknowledges that the relationship among the three types of working memory remains unknown. "While the test battery provides a sound and wide-ranging assessment of the verbal capacities of the CE, it does not tap non-verbal central executive capacities" (Pickering \& Gathercole, 2001, p. 4). Therefore, a set of ANOVAs was appropriate in identifying differences among the three types of working memory between the two groups, but a MANOVA would not have yielded meaningful results.

\section{Confounding Variables}

One confounding variable was that participants could have dropped out and discontinued testing procedures. Another was that there may have been an unconscious bias by the researcher while assessing working memory capacity. Additionally, there may have been unforeseen medical and/or psychiatric issues evident in a group participant after the study began that would have precluded that individual's continued participation. Although measures of assessment and evaluation were utilized prior to the study to identify and eliminate volunteers with comorbid disorders, they were referred to the appropriate care so that treatment issues were addressed. In the present study, no participants were excused because they had comorbid disorders. 
Every effort was made to find children of roughly equal IQ between the two groups to address the research questions (working memory), not related constructs (intelligence). The participants in the present study demonstrated a Full Scale IQ below 115 and above 90.

\section{Participants' Rights}

The participants and their parent/legal guardians were free to withdraw from this study without consequence at any time. Participation was completely voluntary and anyone, with or without disclosing a reason, could have immediately stop participating in the study. Because low social competence can cause embarrassment, shame, frustration, negative sense of self, and a multitude of other problems in many areas of life (Little, 2003) the identities of the study participants remained anonymous. Confidentiality of the assessment data was assured because the participants provided only a code number so that group identification was not revealed to anyone other than the primary researcher. In addition, baseline and assessment data were collected privately and on an individual basis. At no time did any of the participants interact with each other during test administrations or observations.

The WMTB-C and WISC-IV assessments were scheduled to last 100 to 180 minutes. Participants were provided with breaks during the testing sessions. Initially, the researcher had planned to allow the participants to stop the testing procedure before the administration of the test was complete and schedule second session as needed. Dividing the test into two or three sessions did not jeopardize the validity or reliability of the data obtained during the assessment (Pickering \& Gathercole, 2001; Wechsler, 2003). 
However, because the researcher did not encounter participants who wanted to stop the testing procedure, the sessions were not divided.

\section{Summary}

This study sought to identify the mean for each type of IP (i.e., PL, VSSP, and $\mathrm{CE}$ ) within the sample in each group. Each mean was then compared to the mean scores of the sample in the other group using an ANOVA. The research also sought to name the factors that should be considered when developing interventions to mediate deficits in the types of IP skills related to working memory. This study may have intervention program implications. 


\section{CHAPTER 4: RESULTS}

Introduction

This study was designed to identify IP deficits specific to working memory capacity and the impact of AD. The research attempted to determine if differences in working memory capacity exist between boys ages 11.0 to 15.7 diagnosed with $\mathrm{AD}$ and their typically developing, same-age peers. Chapter 4 presents an analysis of the data obtained from this study. Inter-rater reliability data is discussed first, followed by data collected from the sample and an examination of the hypotheses.

$$
\text { Inter-Rater Reliability Data }
$$

Ten raters participated in this study. They signed a confidentiality research assistant form and participated in two training sessions with this researcher to become familiar with the purpose of this study and to review the Gilliam Asperger's Disorder Scale (GADS; Gilliam, 2001) instrument. Rater training included a discussion, a demonstration, and a participation exercise on obtaining an ADQ on the GADS. During the training sessions, all raters watched a DVD entitled, "Diagnosis and Assessment of Asperger's Disorder" (Attwood, 2004), which included an interview with an 11-year-old boy with $\mathrm{AD}$. Each rater observed the boy and recorded observations using the GADS instrument to yield an ADQ score. Inter-rater reliability was established by running a Lin's Concordance Coefficient between each rater (see Table 1). 
Table 1

Inter-rater Reliability for AD Training

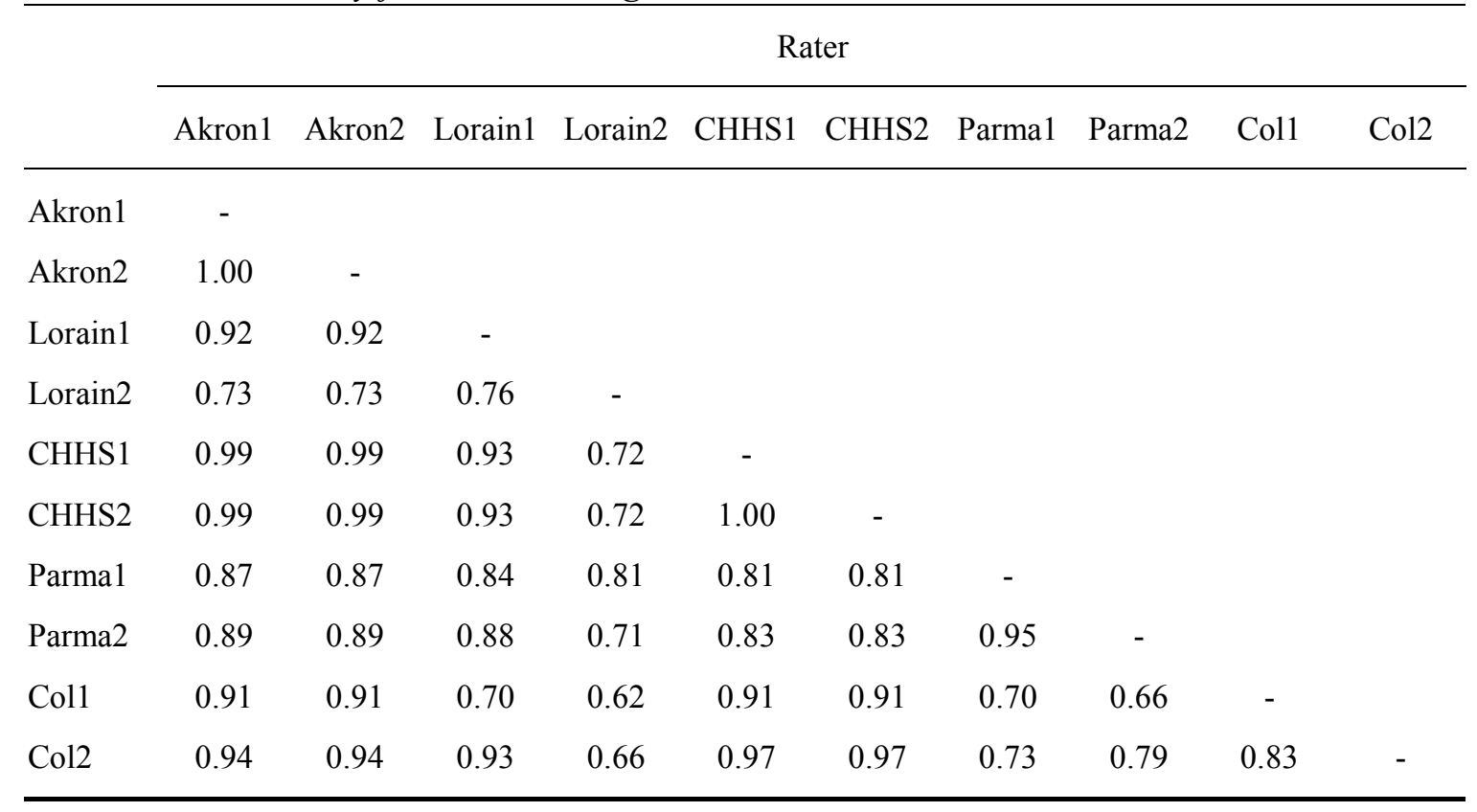

When intercompared, the inter-rater reliability quotients for the 10 raters fell between .62 and 1.00 , with a mean value .86 . Note that this means that there are 45 unique coefficients comparing each of the 10 raters to every other possible rater. These figures suggest that the raters were similarly rating the test individual on the DVD and correctly identifying the symptoms he displayed as indicative of AD. The only possible exceptions to these similar ratings described above were the five pairs of raters who fell below the 0.700 level. Note that Lin's Concordance Coefficient is interpreted under the same guidelines as the better-known Pearson's Correlation Coefficient (r). In fact, the main difference between the two statistics is that Lin's Concordance Coefficient tends to become smaller as the average difference between two objects becomes larger 
(Neuendorf, 2000). As such, one can expect lower Lin's than Pearson's Coefficients for the same set of interrelated objects.

\section{Sample Description}

A total of 76 boys between the ages 11.0 and 15.7 volunteered to participate in the study. Because of the high number of boys who volunteered, the researcher did not have to seek volunteers younger than age 11.0, as originally presumed. The advantage of including subjects who are 11.0 years or older was justified by Gathercole (1998) and Gathercole et al. (2004), who found that as children's frontal lobes develop, so, too, does their capacity for PL. Therefore, according to current developmental research of working memory capacity, the participants in this study met the developmental expectations in terms of their capacity to recode visual and spatial information into verbal phonological form, and they had developed their rehearsal and storing capacities for PL functioning (Gathercole et al.).

Of the original 76 volunteers, 16 individuals did not meet the criteria for participation. Eleven individuals had a Full Scale IQ greater than 115 or below 85, and 5 individuals were rated above 60 and below 95 on the GADS assessment. Therefore, these 16 individuals were omitted from the study. Of the final 60 participants, the mean age was $13.28(S D=1.22)$, and the mean Full Scale IQ ratio was $102.03(S D=7.95)$. The 60 participants were divided equally and assigned to either the AD group (Group A) or the typically developing, same-age peer group (Group T). Group assignment was based on meeting specific criteria, including the presence or absence of a prior diagnosis of AD and a final ADQ score of 95 and above or 60 and below (see Table 2). 
Table 2

Means and Standard Deviations of Select Sample Descriptors for Group A and Group T

\begin{tabular}{ccccccc}
\hline \multirow{2}{*}{ Group } & \multicolumn{3}{c}{ Age } & \multicolumn{3}{c}{ IQ } \\
\cline { 2 - 7 } & $M$ & $S D$ & $M$ & $S D$ & $M$ & $S D$ \\
\hline \multirow{2}{*}{$\mathrm{T}$} & 13.23 & 1.27 & 103.8 & 7.79 & 41.73 & 1.84 \\
$\mathrm{~A}$ & 13.33 & 1.18 & 100.27 & 7.85 & 109.13 & 7.68 \\
\hline
\end{tabular}

Data for Each Group

The age range for Group A was 11.0 to 15.7, with a mean age of 13.33

$(S D=1.18)$. Each participant was administered the WISC-IV (Wechsler, 2003). The 30

boys in Group A had a mean Full Scale IQ of $100.27(S D=7.85)$. To be eligible for

Group A, the participants had to have a prior diagnosis of $\mathrm{AD}$ and had to yield a final

ADQ on the GADS (Gilliam, 2001) of 95 or above when observed and rated by 2 trained raters of this study.

The age range for Group T was 11.0 to 15.5 , with a mean age of 13.23

$(S D=1.27)$. Each participant was administered the WISC-IV (Wechsler, 2003). The 30 boys in Group T had a mean Full Scale IQ of $103.80(S D=7.79)$. To be eligible for group $\mathrm{T}$, the participants must not have had a prior diagnosis of $\mathrm{AD}$ and had to yield a final ADQ on the GADS (Gilliam, 2001) of 60 or below when observed and rated by 2 trained raters of this study.

The average ADQ scores Group A were 109.13 and 41.73 for Group T, with standard deviations of 7.68 and 1.84, respectively. Even with the lower level of 
agreement between the raters, as shown in Table 1, the mean difference in ADQs between the groups was large, as shown in Table 2 . If the level of difference between raters were taken as error, even fairly large errors in ratings would still classify participants as either having $\mathrm{AD}$ or developing typically.

The decision was made post hoc to see if the age and IQ scores were significantly different simply to show parity between the two groups. That is, although the ADQ, PL, VSSP, and CE scores were normed according to age, it was possible that either age or IQ may have had unforeseen interactions with these tests. However, no significant differences were found $(t=-.303$ for age, $t=1.750$ for IQ) between the two groups, so the two groups were assumed to be relatively equal in terms of these two variables. Note that independent $t$ tests were used in gauging these effects simply to check for limitations within the sample, not to prove a conceptual theory.

\section{WMTB-C Data}

The participants from Groups A and T were administered the WMTB-C (Pickering \& Gathercole, 2001). The WMTB-C yielded three working memory scores: PL, VSSP, and CE working memory capacity. The scores were compared using one-way ANOVAs for each DV.

Hypothesis 1

Null Hypothesis 1: There was no difference in PL functioning in children with AD, as compared to typically developing, same-age peers, based on the WMTB-C. Alternative Hypothesis 1: Children with AD demonstrated lower PL functioning compared to typically developing peers based on the WMTB-C. 
The first hypothesis explored the PL capacity of children between the ages of 9 and 15. PL is the ability to process, hold, and store verbal information in terms of sound structure and spoken language (Baddeley et al., 1998). Composite scores on the 4 PL subtests of the WMTB-C (Pickering \& Gathercole, 2001) yielded one PL score. Each subtest yields a standard score that was calculated into the PL score.

An ANOVA was performed to test PL scores and indicated a significant difference between the groups at the $p<.01$ level. A comparison between the scores of the A and T groups yielded an $F$ value of 38.25, which was significant at the $p<.01$ level $(d f 1)$. Because the .05 threshold was exceeded, null hypothesis 1 was rejected, and alternative hypothesis 1 (i.e., the PL capacity of AD individuals was lower in comparison to their typically developing, same-age peers) was accepted. The effect size for this comparison was .397 (i.e., $39.7 \%$ of the variance was explained when comparing condition to PL scores). If this were transformed into a point by serial correlation coefficient (Jaccard \& Becker, 1997), it would be .630 (see Table 3).

Table 3

ANOVA Testing PL Scores between Group A and Group T

\begin{tabular}{llcccc}
\hline & & Sum of & & & \\
& & Squares & $d f$ & Mean Square & $F$ \\
\hline Phonological Loop & Between Groups & 6120.60 & 1 & 6120.60 & $38.25^{* *}$ \\
& Within Groups & 9280.13 & 58 & 160.00 & \\
& Total & 15400.73 & 59 & & \\
\hline
\end{tabular}

$* * p<.01$ 
Hypothesis 2

Null Hypothesis 2: There was no difference between VSSP capacity in children with $\mathrm{AD}$, as compared to typically developing, same-age peers, based on the WMTB-C. Alternative Hypothesis 2: Children with AD demonstrated a lower level of VSSP capacity compared to typically developing, same-age peers, based on the WMTB-C.

The second hypothesis explored the VSSP capacity of children between ages 11 and $15 . \mathrm{VSSP}$ is the capacity to store nonverbal information that has either visual (color, shape) or spatial (movement, relative location) characteristics (Baddeley, 1996; Pickering \& Gathercole, 2001). Composite scores on the VSSP subtests of the WMTB-C and the VPT (Della Sala et al., 1997) yielded one VSSP score. Each subtest yielded a standard score that was calculated into the VSSP score.

An ANOVA to test VSSP scores indicated a significant difference between the groups at the $p<.01$ level. A comparison between the scores of the groups yielded an $F$ value of 22.43, which was significant at the $p<.01$ level $(d f=1)$. Because the .05 threshold was exceeded, null hypothesis 2 was rejected, and alternative hypothesis 2 (i.e., VSSP capacity of AD individuals is lower compared with typically developing, same-age peers) was accepted. The effect size for this comparison was .279 (i.e., $27.9 \%$ of the variance explained when comparing conditions to VSSP scores). If this were transformed into a point by serial correlation coefficient (Jaccard \& Becker, 1997), it would be .528 (see Table 4). 
Table 4

\begin{tabular}{clcccc}
\multicolumn{6}{c}{ ANOVA Testing VSSP Scores between Group A and Group T } \\
\hline & & Sum of & & \\
& Squares & $d f$ & Mean Square & $F$ \\
\hline Visual-Spatial Sketch & Between Groups & 3808.07 & 1 & 3808.06 & $22.43^{* *}$ \\
Pad & Within Groups & 9843.87 & 58 & 169.72 & \\
& Total & 13651.93 & 59 & & \\
\hline
\end{tabular}

$* * p<.01$

Hypothesis 3

Null Hypothesis 3: There was no difference in CE functioning in children with $\mathrm{AD}$ as compared to typically developing, same-age peers based on the WMTB-C.

Alternative Hypothesis 3: Children with AD demonstrated a lower capacity of CE compared to typically developing peers based on the WMTB-C.

The third hypothesis explored the CE capacity of children between ages 9 and 15 . The $\mathrm{CE}$ is responsible for planning and coordinating the flow of information and retrieving knowledge from long-term memory (Baddeley, 1996; Pickering \& Gathercole, 2001). Composite scores on the CE subtests of the WMTB-C yielded one CE score. Each subtest yielded a standard score that was calculated into the CE score.

An ANOVA performed to test CE scores indicated a significant difference between the groups at the $p<.01$ level. A comparison between the scores of the groups yielded an $F$ value of 97.50, which was significant at the $p<.01$ level ( $d f 1)$. Because the .05 threshold was exceeded, null hypothesis 3 was rejected, and alternative hypothesis 3 (i.e., children with $\mathrm{AD}$ demonstrated a lower capacity of CE compared to typically developing peers based on the WMTB-C) was accepted. The effect size for this comparison was .627 (i.e., $62.7 \%$ of the variance was explained when comparing 
condition to $\mathrm{CE}$ scores). If this were transformed into a point biserial correlation coefficient (Jaccard \& Becker, 1997), it would be .799 (see Table 5).

Table 5

ANOVA Testing CE Scores between Group A and Group T

\begin{tabular}{llcccc}
\hline & & Sum of & & & \\
& Squares & $d f$ & Mean Square & $F$ \\
\hline Central Executive & Between Groups & 13320.60 & 1 & 13320.60 & $97.50^{* *}$ \\
& Within Groups & 7923.73 & 58 & 136.67 & \\
& Total & 21244.33 & 59 & & \\
\hline
\end{tabular}

$* * p<.01$

Summary of Results

Chapter 4 evaluated the working memory capacity of 60 boys between the ages of 11.0 and 15.7 and compared the verbal (PL), visual-spatial (VSSP), and complex memory (CE) scores of 30 individuals diagnosed with $\mathrm{AD}$ and 30 typically developing, same-age peers. The research data indicated that individuals with AD have a significantly lower working memory capacity in comparison to their typically developing peers in all three areas of working memory. The effect sizes between Groups A and T for PL, VSSP, and CE were $.397, .279$, and .627, respectively, when measured by Eta ${ }^{2}$ (see Table 6).

Table 6

PL, VSSP, and CE Score Means, Standard Deviations, and Effect Sizes for Group A and Group T

\begin{tabular}{|c|c|c|c|}
\hline & \multicolumn{2}{|c|}{ Group } & \multirow{2}{*}{ Effect Size** } \\
\hline & $\mathrm{T}$ & A & \\
\hline PL & $103.53(12.17)^{*}$ & $83.33(13.11)$ & 0.397 \\
\hline VSSP & $86.00(13.39)$ & $70.07(12.65)$ & 0.279 \\
\hline $\mathrm{CE}$ & $104.07(11.59)$ & $74.27(11.79)$ & 0.627 \\
\hline
\end{tabular}

$* S D$ s are in parentheses.

**Effect size was measured with Eta ${ }^{2}$ (Between divided by Total Variance). 
The closer an effect size gets to 1.00 , the stronger the condition is on the measured score (Jaccard \& Becker, 1997), indicating the degree of influence a condition has on a measurement. The effect sizes in this study indicated that the strongest relationship the condition has to any of these scores was the CE score, and the weakest relationship was the VSSP score.

Chapter 5 summarizes the results from this study and discusses the results obtained in reference to the research literature. Implications for social change are presented, along with suggestions for potential therapeutic interventions when working with individuals with AD who demonstrate poor social skill functioning. Finally, recommendations for future research are made. 


\section{CHAPTER 5: SUMMARY, CONCLUSIONS, AND RECOMMENDATIONS \\ Introduction}

This chapter provides a synopsis of the study, a review of the hypotheses, and a discussion of this study's findings in relation to current literature and theoretical frameworks relevant to working memory and social functioning. The discussion of the study offers emerging issues and themes that resulted from the data analysis. Implications for social change concerning interventions that may improve working memory skills that contribute to the development of social skill competence are presented. Finally, the study's limitations are addressed, and recommendations for future research are offered.

Researchers (e.g., Bauer, 1996; Carter et al.; 2004; Gutstein \& Whitney, 2002; Molloy \& Vasil, 2002) have concluded that children with AD have IP deficits and lack the ability to utilize nonverbal social cues, regulate social interactions, and engage in social or emotional reciprocity. These social tasks require the simultaneous processing of PL, VSSP, and CE information in "real time," that is, the individual must utilize working memory skills. The current study investigated the working memory capacity of AD individuals, focusing on IP deficits specific to the ability to interpret and respond to nonverbal and verbal information inherent in social relationships.

\section{Overview of the Current Study}

The study compared three specific working memory capacities (verbal [PL], visual-spatial [VSSP], and complex [CE]) of 30 males ages 11.0-15.7 years with AD to those same capacities in 30 typically developing, same-age peers. The researcher hypothesized that there would be significant differences in the PL, VSSP, and CE 
working memory capacities of the two groups. As discussed in chapter 4 , the data analysis supported the three alternative hypotheses. The working memory capacities of the children with $\mathrm{AD}$ were significantly lower than those demonstrated by the typically developing, same-age peers. The findings are interpreted in the next section.

Interpretation of the Findings

PL

The finding that the PL capacity of individuals with AD is lower than that of their typically developing, same-age peers contributes to a better understanding of the cognitive skills demonstrated by individuals with AD. The effect size was meaningful when comparing condition to PL scores and indicated that $39 \%$ of the variance was explained. This finding supported the theory that social skill deficits relate to verbal and auditory cognitive processes (Gutstein \& Whitney, 2002) but that the specific cognitive deficits have yet to be identified. Current literature on the cognitive skills of individuals with AD has shown that they perform inconsistently on the WISC-IV (Wechsler, 2003) and have no significant differences between verbal and performance IQs (Barnhill et al., 2000). Adams et al. (2002) found that individuals with AD do not demonstrate an overall deficit in verbal memory or verbal knowledge.

However, why are individuals with $\mathrm{AD}$ who demonstrate strengths in verbal cognitive skills, verbal knowledge, and verbal comprehension unable to apply this information to everyday social tasks (Barnhill et al., 2002)? The finding that the PL capacity of individuals with $\mathrm{AD}$ is lower than that of their typically developing, same-age peers revealed an important relationship between PL and cognitive functioning, and it 
may explain why many $\mathrm{AD}$ individuals who demonstrate strengths in verbal skills and verbal knowledge cannot utilize verbal information effectively to perform everyday social tasks.

$\mathrm{PL}$ is responsible for processing, coding, remembering, and storing verbal information in terms of sound structure, sequences, and spoken language (Baddeley et al., 1998; Pickering, 2001a). It is utilized during social situations to temporarily store, manipulate, and respond appropriately to the ongoing social activity (Gathercole \& Pickering, 2001). Working memory allows the individual to process PL during a novel social situation so that a higher level of reasoning is achieved (Baddeley, 1996). The IP skills specific to working memory capacity are more complex than the rote memory skills that are dependent on a unitary structure of memory (Baddeley; DiLollo \& Dixon, 1992, 1998; Gathercole, 1998; Shulman, 1971).

As discussed in chapter 2, short-term rote memory tasks assessed by the subtests of the WISC-IV (Wechsler, 2003) do not consider memory task performance that requires the simultaneous manipulation of multiple types of information (Bayliss et al., 2003; Kane \& Engle, 2000; Shah \& Miyake, 1996). Therefore, the working memory deficits demonstrated by the boys with $\mathrm{AD}$ in this study contributed to the research of Barnhill et al. (2002) and Marshall (2002), who concluded that although individuals with AD have good rote memory and verbal comprehension skills, they cannot transfer these skills to a social context. Barnhill et al. explained that social skill instruction that incorporates role-playing, videotaping, and game-playing increases the self-confidence of AD individuals, but the skills learned during this instruction do not generalize to home, 
school, and community settings. They stated, "Clearly, more research on social skill instruction is needed for individuals with AD and other autism spectrum disabilities" (p. 115).

The present study reinforces the need to investigate current social instructional interventions and differentiate between interventions that focus on rote memory (simple span) tasks and working memory (complex span) tasks. Verbal knowledge and verbal rote memory may not be deficit when individuals with $\mathrm{AD}$ are cognitively assessed with the WISC-IV or when they perform rote memory social tasks during social skill group interventions. In fact, these skills may not be deficit. Instead, as suggested in the current study, the working memory capacity of individuals with AD may be the specific IP deficit that is more important to the lack of social skill development than the ability to recall already learned verbal information or situation-based social instructions.

VSSP

The finding that AD individuals have a lower capacity for VSSP than their typically developing, same-age peers is congruent with the right hemispheric deficit hypothesis and the social deficit hypothesis, demonstrating that children with AD miss or fail to interpret nonverbal social cues (Gustein \& Whitney, 2002). Impairments in processing nonverbal information include the inability to decipher such nonverbal cues as tone of voice, gestures, nuances, and facial expressions (Barnhill et al., 2002; Ghaziuddin \&Mountain-Kimchi, 2004; Gunter et al., 2002; Gustein \& Whitney; Koning \& MaGillEvans, 2001; Little, 2003). 
According to the right hemispheric deficit hypothesis, when right brain functioning is impaired, the individual lacks the ability to interpret nonverbal cues within a social situation and to learn age-appropriate social interactions. Right brain functioning impairment results in problems with visual-spatial perception, visual memory, and nonverbal concept formation (Casanova, Buxhoeveden, Switala, \& Emil, 2002; Gunter et al., 2002; Gutstein \& Whitney, 2002; Rourke et al., 2002). VSSP capacity directly relates to right hemispheric functioning (Smith \& Jonides, 1999) and is analogous to working memory capacity and the ability to understand and manipulate nonverbal information. VSSP, "situated in the right hemisphere" (Pickering \& Gathercole, 2001, p. 2), is responsible for processing, coding, remembering, and storing nonverbal information that has either visual (color, shape) or spatial (movement, relative location) characteristics (Pickering, \& Gathercole).

Therefore, according to the results of the present study, children with AD who demonstrate a low capacity for VSSP most likely have difficulty processing nonverbal visual and spatial information inherent in social interactions. This IP deficit impairs reciprocal social communication skills (Leiberman \& Rosenthal, 2001). Although the weakest effect size occurred when comparing condition to VSSP scores, this effect size demonstrated that a meaningful relationship exists, and explained a $27 \%$ variance in scores. This supported the finding that people rely on nonverbal cues two times as often when interacting with interpersonal targets (Leiberman \& Rosenthal). 
$C E$

Interestingly, the largest effect size was seen when comparing condition to $\mathrm{CE}$ scores. The effect size showed that a meaningful relationship exists and also explained the $62.7 \%$ variance in of the complex memory scores between groups A and $\mathrm{T}$.

This finding is consistent with the social attention deficit theory that proposes that an individual has difficulty attending to multiple social cues (verbal, spatial and visual) while waiting to respond to the spontaneous and novel social interaction. The finding that $\mathrm{AD}$ individuals appear to have a reduced capacity for complex working memory may further clarify the connection between information processing deficits of social attention and the underlying cognitive processes that directly relate to working memory capacity.

This finding is also consistent with the theory of mind (TOM) deficit proposed by Baron-Cohen and Hammer (1997) and Frith and Happé (1999), who posited that individuals with AD lack the ability to "mind read" or surmise the desires, intensions, beliefs, and mental states of the self or others. As discussed in chapter 2, TOM, which supports the social deficit hypothesis, require two levels of IP: (a) differentiating among beliefs, pretend knowledge, and real knowledge (Lawson, 2003), and (b) understanding how to use this information (Attwood, 2004). TOM skills require the dual processing of nonverbal and verbal information simultaneously (Little, 2003) and rapidly in real time (Stone, Baron-Cohen \& Knight, 1998) while utilizing working memory functions to code, store, attend to, organize, and retrieve information from memory (Baddeley, 2002b; Gathercole \& Pickering, 2001). 
Dual processing tasks, by definition, require $\mathrm{CE}$ functioning and the ability to simultaneously process multiple types of information. An example of a dual processing task within a social context is the ability to recognize a faux pas. As mentioned previously, Baron-Cohen et al. (1999) found that individual with AD lack the ability to identify faux pas during social interactions. The IP skills needed to spontaneously detect and respond to faux pas involve the ability to process novel complex IP tasks and simultaneously organize relevant verbal and nonverbal information, capacities directly related to CE functioning (Baddeley, 2003; Bull et al., 1999).

CE has multiple roles in IP: inhibiting irrelevant information from interfering with task performance; updating information; switching between tasks while evaluating new strategies; and maintaining relevant, problem-solving strategies relevant to the task (Bull \& Scerif, 2003). Therefore, it makes sense that individuals with low CE capacity would also demonstrate low abilities to accomplish TOM skills. Interestingly, the largest effect size (.799) occurred when comparing condition to CE scores. According to Barrett et al. (2004), low CE functioning may influence one's ability to suppress previously learned information and determine the meaning of ambiguous affective and social cues. They stated, "The central executive aspect of working memory has had a tremendous influence in cognitive psychology over the past several decades, but is only recently being considered in other areas of psychology theory and research" (p. 585). The findings of the present study may promote future research that investigates the role of CE capacity with regard to the IP skills required to engage in social situations. 
Summary of Implications

The findings demonstrated that the PL, VSSP, and CE capacity of boys with AD between ages 11.0 and 15.7, when compared to that of typically developing, same-age peers may be impaired. The IP skills required to engage in reciprocal social exchanges and to understand the global content of nonverbal and verbal information is fundamentally related to working memory capacity. The findings of this study support the right hemisphere deficit theory, the social deficit theory, and TOM.

The present study demonstrates the need to investigate further the IP deficiencies specific to working memory development and to reconsider the social behavioral interventions of individuals with AD. If working memory development were better understood, interventions could be identified and coordinated to target natural developmental time lines with regard to emerging working memory skills. Remedial efforts could build on existing working memory capacity and target specific working memory skills such as rehearsing, chunking, and manipulating verbal, visual and spatial cues that occur simultaneously.

\section{Implications for Social Change}

Studies in evolutionary anthropology have found that it is unnatural for humans to live in isolation from a social group (Dunbar, 2003); that social exchange is universal (Nettle \& Dunbar, 1997); and that memory is a prominent feature of reciprocal social behavior (Cox et al., 1999). These findings are congruent with current literature on AD that because the IP deficiencies cause stress and anxiety (Attwood, 2004; Little, 2003), the unnatural social isolation experienced by individuals with AD is undesirable. 
Everyday social difficulties last throughout the life span; therefore, individuals with AD have difficulty making friends; understanding social rules (Gustein \& Whitney, 2002); and deciphering nonverbal nuances of social exchanges (Little). They are perceived by adults and peers as outcasts from the group (Attwood; Carter et al., 2004).

Social interaction skills and social competence have repeatedly been viewed as critical to the ability of individuals with AD to succeed in academic, work, social, family, and emotional relationships (Denham et al., 2003). AD affects adults and children of all races, genders, and cultures; therefore, social change in relation to improving IP skills that relate to working memory and enhanced social interactions is immeasurable. Increasing the ability to process complex social information will have a direct impact on almost every aspect of life for individuals with AD by decreasing the experiences of isolation, dependence, and peer rejection. Instead, having the ability to process information needed to engage in appropriate social interactions will allow individuals with $\mathrm{AD}$ to participate in social peer groups and to experience intimacy, social acceptance, and independence as productive adults. When this occurs, positive social change is inevitable.

\section{Recommendations for Action}

Parents, teachers, therapists, and individuals with AD can begin to teach themselves how working memory is related to social skill functioning. Psychologists can provide accurate assessments for individuals with $\mathrm{AD}$ and focus on working memory capacity deficiencies and strengths while looking specifically at the social skill deficiencies presented by individuals with AD. The findings from the current study 
suggest that it may be advantageous to clinical and neurological psychologists to investigate the limitations of the working memory capacity of individuals with AD so that they can understand the cognitive processes that underpin a reduced capacity to demonstrate social skills.

Strengths in working memory capacity can be utilized to remediate challenges in working memory capacity so that the individual builds on existing working memory skills. By distributing information about the impact of working memory development to parents through workshops, parent publications and internet resources, they can gain an understanding of working memory deficiencies and development to promote an increase of working memory capacity through remedial efforts for AD children. Working memory techniques could be applied to structured and unstructured group situations in which individuals with AD can begin to identify, understand, and apply working memory techniques to verbal and nonverbal social cues. They may gain a sense of control over their treatment plans, encouraging social connections by understanding specific tangible exercises targeted directly at specific IP deficiencies.

Implications for Future Research

Future research should continue to investigate how working memory capacity relates to individuals with $\mathrm{AD}$ by conducting rigorously designed and controlled studies that address specific social skill deficiencies. The present study utilized a previous diagnosis of $\mathrm{AD}$ and an average $\mathrm{ADQ}$ score from two assigned raters to ensure that each participant in Group A met the criteria for group placement. Although this procedure ensured clinical and statistical differences between Groups A and T, the current study did 
not look specifically at the subcategories identified by the GADS instrument: social interaction, restricted patterns of behavior, cognitive patterns, and pragmatic skills. It may be advantageous for future studies to compare the ratings of the subcategories of each individual $\mathrm{AD}$ with their specific working memory capacity scores. In this way, working memory capacity can be examined within the context of the specific social deficiencies of individuals with AD.

In the present study, participants were at least 11 years old, and, according to current research, had developed PL techniques to retain information (Pickering \& Gathercole, 2001). It would be worthwhile to investigate the working memory capacities of younger children with AD who depend more on VSSP to engage in everyday activities. By assessing the working memory functions of children with AD of different age groups, the developmental course of working memory functions can be examined to clarify the nature and extent of limitations that may influence the development of social skill functioning. Additionally, girls were not included in the present study. Future studies can expand on the investigation of individuals with AD and compare the working memory capacities of boys to girls. Also, future research can compare the working memory capacities of individuals with AD to other populations, such as individuals with attention deficit disorder, autism, or other PDDs.

Future research could investigate the effectiveness of remedial programs that teach working memory techniques: chunking, articulatory rehearsal, information manipulation, and selective attending to relevant details and the visual-spatial scanning of nonverbal cues. These techniques were identified in the literature as necessary for 
effective IP specific to working memory (Baddeley, 2002b; Mueller et al., 2003;

Pickering et al., 2001). Because the basic constructs of the working memory model are easily testable and the brain basis of these functions is understood (Baars \& Franklin, 2003), specific IP deficiencies related to working memory capacity can be identified and assessed. Future studies can examine whether or not increasing the working memory techniques of individuals with $\mathrm{AD}$ has a positive affect on social skill development and whether an increase of working memory capacity in one area affects an increase in the processing of social information. For example, can an individual who improves CE skills more effectively process multiple types of information simultaneously (verbal, visualspatial, and complex) within a novel social situation? Will the individual who improves CE skills also improve TOM skills or social attention skills? The results of the present study has uncovered evidence to substantiate that future research examine the affect of improving the working memory capacity of individuals with AD to further clarify specific cognitive and social deficiencies related to working memory.

Future research can also examine AD individuals who do not demonstrate deficiencies in working memory capacities and compare their social functioning to those AD individuals who do. Comparisons such as this can identify developmental factors of working memory or other variables in IP that impair social functioning.

The present study offered significant value by demonstrating that IP skills related to working memory is deficit in individuals with AD. Further exploration of this IP deficiency may contribute to an understanding of the difference between the ability of 
individuals with $\mathrm{AD}$ to perform learned social skills in a structure environment and their inability to generalize those skills to a novel social situation.

Consistent findings of current literature is that the efficacy of contemporary social-behavioral skill training of individuals with $\mathrm{AD}$ is poor because it is situation based, not easily generalized to novel or real-life situations outside of the educational or therapeutic environment, and not successful long term (Dyck et al., 2001; Parsons \& Mitchell, 2002). Strong rote-memory scores on tests of comprehension “do not necessarily reflect the ability to apply this rote knowledge in practical, everyday social situations" (Barnhill et al., 2000, p. 152).

Summary

The study justified the need to reassess treatment interventions for individuals with AD. Reflecting Parsons and Mitchell's (2002) insight, the researcher asks the following question: Would social skill training that focuses on the development of working memory be more effective than current social skill training that focuses on the practice and memory of situation-based social and behavioral gestures? The findings of the present study suggest that AD individuals have IP deficiencies specific to working memory (i.e., PL, VSSP, and CE). Therefore, innovative working memory interventions may be more effective in the long term because they focus on the development of IP skills rather than situation-based behaviors and rote memory learning. After examining the readily available literature pertaining to individuals with AD, IP and the WMTB-C, this study was the first to combine theories from cognitive, neurological, and clinical psychology; isolate specific working memory components; and identify a connection 
between working memory capacity and the social skill deficiencies of individuals with AD. The present study has heuristic value that can lead future research to expand upon this connection and more clearly understand the IP deficiencies related to working memory. 


\section{REFERENCES}

Adams, C., Green, J. Gilchrist, A., \& Cox, A. (2002). Conversational behavior of children with Asperger syndrome and conduct disorder. Journal of Child Psychology and Psychiatry, 43, 679-690.

American Psychiatric Association. (2000). Diagnostic and statistical manual of mental disorders ( $4^{\text {th }}$ ed.). Washington DC: Author.

American Psychological Association. (2001). The principles of medical ethics with annotations especially applicable to psychiatry. AMA Council of Ethical and Judicial Affairs. Washington, DC: Author.

Attwood, T. (1998). The links between social stories, comic strip observations and the cognitive models of autism and Asperger's syndrome. Retrieved July 16, 2004, from http://www.tonyattwood.com.au/paper5.htm

Attwood, T. (2004). Dr. Tony Attwood presents: Asperger's diagnostic assessment. Future Horizons. New York: Arlington.

Awh, E., Jonides, J., Smith, E., Schumacher, E., Koeppe, R., \& Katz, S. (1996). Dissociation of storage and rehearsal in verbal working memory: Evidence from Positron Emission Tomography. Psychological Science, 7, 25-31.

Baars, B., \& Franklin, S. (2003). How conscious experience and working memory interact, Trends in Cognitive Science, 48, 1-7.

Baddeley, A. (1966). Short-term memory for word sequences as a function of acoustic, semantic and formal similarity, Quarterly Journal of Experimental Psychology, $18,362-365$.

Baddeley, A. (1996). The fractionation of working memory. Proceedings of the National Academy of Science of the United States of America, 93, 1346813473.

Baddeley, A. (2001). Alan D. Baddeley. American Psychologist, 56, 849-851.

Baddeley, A. (2002a). Fractionating the central executive. Neuroscience, 7, 246-260.

Baddeley, A. (2002b). Is working memory still working? European Psychologist, 7, 85-77.

Baddeley, A. (2003). Working memory: Looking back and looking forward, Neuroscience, 4, 829-839. 
Baddeley, A., Gathercole, S., \& Papagno, C. (1998). The phonological loop as a language learning device. Psychological Review, 105, 158-173.

Baddeley, A., \& Hitch, G. (1974). Working memory. In E. G. Bower (Ed.), The psychology of learning and motivation (pp. 47-89). New York: Academic Press.

Baddeley, A., \& Logie, R. (1999). Working memory: The multiple component model. In Miyake \& P. Shah (Eds.), Models of working memory (pp. 28-61). New York: Cambridge University Press.

Baddeley, A., \& Wilson, B. (1985). Phonological coding and short-term memory in patients without speech. Journal of Memory and Language, 24, 490-502.

Barnhill, G., Cook, K., Tebbenkamp, K., \& Myles, B. (2002). The effectiveness of social skills intervention targeting non-verbal communication for adolescents with Asperger syndrome and related pervasive developmental delays. Focus on Autism and Other Developmental Disabilities, 17, 112-119.

Barnhill, G., Hagiwara, T., Myles, B., \& Simpson, R. (2000). Asperger syndrome: A study of the cognitive profiles of 37 children and adolescents. Focus on Autism and Other Developmental Disabilities, 15, 146-153.

Baron-Cohen, S. (1989). The autistic child's theory of mind: A case of specific developmental delay. Journal of Child Psychology and Psychiatry and Allied Disciplines, 30, 285-298.

Baron-Cohen, S. (1995). Mind-blindness: An essay on autism and theory of mind. Cambridge, MA: MIT Press.

Baron-Cohen, S., \& Hammer, J. (1997). Parents of children with Asperger syndrome: What is the cognitive phenotype? Journal of Cognitive Neuroscience, 9, 548-554.

Baron-Cohen, S., Jolliffe, T., Mortimore, C., \& Robertson, M. (1997). Another advanced test of theory of mind: Evidence from very high-functioning adults with autism or Asperger syndrome. Journal of Child Psychology \& Psychiatry, 38, 813-822.

Baron-Cohen, S., O’Riordan, M., Stone, V., Jones, R., \& Plaisted, K. (1999). Recognition of faux pas by normally developing children and children with Asperger syndrome or high-functioning autism. Journal of Autism and Developmental Disorders, 29, 407-418. 
Baron-Cohen, S., Wheelwright, S., Hill, J., Raste, Y., \& Plumb, I. (2001). The "Reading the Mind in the Eyes" test revised version: A study with normal adults, and adults with Asperger syndrome or high-functioning autism. Journal of Child Psychology and Psychiatry, 42, 241-251.

Barrett, L. Tugade, M., \& Engle, R. (2004). Individual differences in working memory capacity and dual-process theory of mind. Psychological Bulletin, 130, 553-573.

Bauer, S. (1996). Asperger syndrome. O.A.S.I.S., $x x, 1-10$.

Bauminger, N., \& Kasari, C. (2000). Loneliness and friendship in high-functioning children with autism. Child Development, 71, 447-460.

Bayliss, D., Jarrold, C., Gunn, D., \& Baddeley, A. (2003). The complexities of complex span: Explaining individual differences in working memory in children and adults. Journal of Experimental Psychology, 132, 71-92.

Bellini, S. (2004). Social skill deficit and anxiety in high functioning adolescents with autism spectrum disorders. Focus on Autism and Other Developmental Disabilities, 19, 78-86.

Bonnet, K., \& Gao, X. (1996). Asperger's syndrome in neurologic perspective. Journal of Child Neurology, 6, 483-489.

Bull, R., Johnston, R., \& Roy, J. (1999). Exploring the roles of the visual-spatial sketch pad and central executive in children's arithmetical skills: Views from cognition. Developmental Neuropsychology, 15(3), 421-445.

Bull, R., \& Scerif, G. (2001). Executive functioning as a predictor of children's mathematics ability: Inhibition, switching and working memory. Developmental Neuropsychology, 19, 273-292.

Caplan, D., Rochon, E., \& Waters, G. S. (1992). Articulatory and phonological determinants of word length effects in span tasks. Quarterly Journal of Experimental Psychology, 44A, 177-192.

Carrington, S., Templeton, E., \& Papinczak, T. (2003). Adolescents with Asperger's syndrome and perceptions of friendship. Focus on Autism and Other Developmental Disabilities, 18, 211-218.

Carter, C., Meckes, L., Pritchard, L., Swensen, S., \& Wittman, P. (2004). The friendship club: An after school program for children with Asperger's syndrome. Family Community Health, 27, 143-150. 
Casanova, M., Buxhoeveden, D., Switala, A., \& Emil, R. (2002). Asperger's syndrome and cortical neuropathology. Journal of Child Neurology, 17, 142-150.

Case, R. (1992). The role of the frontal lobes in the regulation of cognitive development, Brain and Cognition, 20, 51-73.

Chelune, G., \& Baer, R. (1986). Developmental norms for the Wisconsin Card Sorting Test. Journal of Clinical and Experimental Neuropsychology, 8, 219-228.

Clark, T., Feehan, C., Tinline, C., \& Vostanis, P. (1999). Autistic symptoms in children with attention deficit hyperactivity disorder. European Child and Adolescent Psychiatry, 8, 50-57.

Cohen, I., Schmidt-Lackner, S., Romanczyk, R., \& Sudhalter, V. (2003). The PDD Behavior Inventory: A rating scale for assessing response to intervention in children. Pervasive Developmental Disorder, 33, 31-45.

Conners, F., Atwell, J. Rosenquist, C., \& Sligh, A. (2001). Abilities underlying decoding differences in children with intellectual disability. Journal of Intellectual Disability Research, 45, 292-301.

Conrad, R., \& Hull, A. (1964). Information acoustic confusion and memory span. British Journal of Psychology, 55, 429-432.

Conway, A., \& Engle, W. (1996). Individual difference in working memory capacity: More evidence for a general capacity theory. Memory, 4, 577-590.

Corcoran, R., \& Mercer, G. (1995). Schizophrenia symptomatology and social influence: Investigating "theory of mind" in people with schizophrenia. Schizophrenia Research, 17, 5-13.

Cox, S., Sluckin, T., \& Steele, J. (1999). Group size, memory and interaction rate in the evolution of cooperation. Current Anthropology, 369-377.

Craig, J., \& Baron-Cohen, S. (1999). Creativity and imagination in autism and Asperger's syndrome. Journal of Autism and Developmental Disorders, 29, 319-326.

Daneman, M., \& Carpenter, P. (1980). Individual differences in working memory and reading. Journal of Verbal Learning and Verbal Behavior, 19, 450-466.

Daneman, M., \& Carpenter, P. (1983). Individual difference in integrating information between and within sentences. Cognition, 9, 561-584. 
Daneman, M., \& Merikle, P. (1996). Working memory and language comprehension: A meta-analysis. Psychonomic Bulletin \& Review, 3, 422-433.

David, R., David, C., \& Riley, L. (2003). Asperger's and related disorders. Practical Neurology, 3, 150-159.

Dawson, G., Meltzoff, A., Osterling, J., Rinaldi, J., \& Brown, E. (1998). Children with autism fail to orient to naturally occurring stimuli. Journal of Autism and Developmental Disorders, 28, 479-485.

Dawson, G., Toth, K., Abbott, R., Osterling, J., Munson, J., Estes, A., et al. (2004). Early social attention impairments in autism: Social orienting, joint attention and attention to stress. Developmental Psychology, 40, 271-283.

Della Sala, S., Gray, C., Baddeley, A., \& Wilson, L. (1997). Visual patterns test: A test of short-term visual recall. Bury St. Edmunds, Suffolk, UK: ThamesValley Test Company.

Denham, S., Blair, K., DeMulder, C., Levitas, J., Sawyer, K., Auerbach-Major, S., et al. (2003). Preschool emotional competence: Pathway to social competence? Child Development, 74, 238-256.

D’Esposito, M., Detre, J., Alsop, D., Shin, R., Atlas, S., \& Grossman, M. (1995). The neuro basis of the central executive system of working memory. Nature, 378 , 279-281.

DiLollo, V., \& Dixon, P. (1988). Two forms of persistence in visual information processing. Journal of Experimental Psychology, 14, 671-681.

DiLollo, V., \& Dixon, P. (1992). Is the icon's worth apples and oranges? Some fruitful thoughts on Loftus, Duncan \& Gehrig. Journal of Experimental Psychology, Human Perception and Performance, 18, 550-555.

Duff, S., \& Logie, R. (2001). Processing and storage in working memory span. Quarterly Journal of Experimental Psychology, 54(A), 31-48.

Dunbar, R. (1992). Neocortex size as a constraint on group size in primates. Journal of Human Evolution, 20, 469-493.

Dunbar, R. (2003). The social brain: Mind, language and society in evolutionary perspective. Annual Reviews, 32, 163-181.

Dunbar, R. (2004). Gossip in evolutionary perspective. Review of General Psychology, 8, $100-110$. 
Dutke, S., \& Stober, J. (2001). Test anxiety, working memory and cognitive performance: Supportive effects of sequential demands. Cognition and Emotion, 15, 381-389.

Dyck, M., Ferguson, K., \& Shochet, M. (2001). Do autism spectrum disorders differ from each other and from non-spectrum disorders on emotional recognition tests? European Child and Adolescent Psychiatry, 10, 105-122.

Eisenmajer, R., Prior, M., Leekam, S., Wing, L. Ong. B., Gould, J., et al. (1998). Delayed language onset as a predictor of clinical symptoms in pervasive developmental disorders. Journal of Autism and Developmental Disorders, 28, 527-533.

Engle, R. W., Kane, M. J., \& Tuholski, S. W. (1999). Individual differences in working memory capacity and what they tell us about controlled attention, general fluid intelligence and functions of the prefrontal cortex. In A. Miyake \& P. Shah (Eds.), Models of working memory: Mechanisms of active maintenance and executive control (pp. 102-134). New York: Cambridge University Press.

Entwisle, D., \& Huggins, W. (1973). Iconic memory in children. Child Development, 44, 392-394.

Falk-Ross, F., Iverson, M., \& Gilbert, C. (2004). Teaching and learning approaches for children with Asperger's syndrome. Counseling for Exceptional Children, 36, 4855.

Farah, M., Hammond, K., Levine, D., \& Calvanio, R. (1988). Visual and spatial mental imagery: Dissociable systems of representation. Cognitive Psychology, 20, 439462.

Filipek, P. A., Accardo, P. J., Baranek, G. T., Cook, E. H., Jr., Dawson, ,G., Gordon, G., et al. (1999). The screening and diagnosis of autistic spectrum disorders. Journal of Autism and Developmental Disorders, 29(6), 439-484.

Freeman, B., Caonin, P., \& Candela, P. (2002). Asperger's syndrome or autistic disorder? The diagnostic dilemma. Focus on Autism and Other Developmental Disabilities, $17,145-151$.

Fombonne, E. (2003). Epidemiological surveys of Autism and other pervasive developmental disorders: An update. Journal of Autism and other Developmental Disorders, 33, 365-382.

Frith, U. (1989). Autism: Explaining the enigma. Oxford: Basil Blackwell. 
Frith, U., \& Happé, F. (1999). Theory of mind and self-consciousness: What is it like to be autistic? Mind and Language, 14, 1-22.

Gathercole, S. (1998). The development of memory. Journal of Psychology and Psychiatry, 39, 3-27.

Gathercole, S. (1999). Cognitive approaches to the development of short-term memory. Trends in Cognitive Science, 3, 410-418.

Gathercole, S., \& Pickering, S. (1999). Estimating the capacity of phonological shortterm memory. International Journal of Psychology, 34, 378-382.

Gathercole, S., \& Pickering, S., (2001). Working memory deficits in children with special educational needs. British Journal of Special Education, 28, 89-96.

Gathercole, S., Pickering, S., Ambridge, B., \& Wearing, H. (2004). The structure of working memory from 4 to 15 years of age. Journal of Developmental Psychology, 40, 177-190.

Gathercole, S., Pickering, S., Knight, C., \& Stegmann, Z. (2003). Working memory skills and educational attainment: Evidence from national curriculum assessment at 7 and 14 years of age. Applied Cognitive Psychology, 18, 1-16.

Gegenfurtner, K., \& Sperling, G. (1993). Information transfer in iconic memory experiments. Journal of Experimental Psychology, 19, 845-866.

Ghaziuddin, M., \& Mountain-Kimchi, K. (2004). Defining the intellectual profile of Asperger syndrome: Comparison with high-functioning autism. Journal of Autism and Developmental Disorders, 34, 279-285.

Gilchrist, A., Green, J., Cox, A., Burton, D., Rutter, M., \& LeCouteur, A. (2001). Development and current functioning in adolescents with Asperger's syndrome: A comparative study. Journal of Child Psychology and Psychiatry, 42, 227-240.

Gilliam, J. (2001). Gilliam Asperger's disorder scale: Examiner's manual. Austin, TX: Pro-Ed.

Gilotty, L., Kenworthy, L., Sirian, L., Black, D., \& Wagner, A. (2002). Adaptive skills and executive function in autism spectrum disorders. Child Neuropsychology, 8, 241-248.

Goldman-Rakic, P. (1996). Regional and cellular fractionation of working memory. Public Library of Science, 93, 13473-13480. 
Greenham, S. (1999). Learning disabilities and psychosocial adjustment: A critical review. Child Neuropsychology, 5, 171-196.

Gunter, H., Ghaziuddin, M., \& Ellis, H. (2002). Asperger's syndrome: Test of right hemisphere functioning and inter-hemispheric communication. Journal of Autism and Developmental Disorders, 32, 263-281.

Gutstein, S., \& Whitney, T. (2002). Asperger syndrome and the development of social competence. Focus on Autism and Other Developmental Disabilities, 17, 161175.

Hadwin, J., Baron-Cohen, S., Howlin, P., \& Hill, K. (1997). Does teaching theory of mind have an effect on the ability to develop conversation in children with Autism? Journal of Autism and Other Developmental Disorders, 27, 519-537.

Hair, J., Anderson, R., Tatham, R., \& Black, W. (1998). Multivariate data analysis (5 ${ }^{\text {th }}$ ed.). Upper Saddle River, NJ: Prentice Hall.

Hanley, J., Young, A., \& Pearson, N. (1991). Impairment of the visuo-spatial sketch pad. Quarterly Journal of Experimental Psychology, 43, 101-125.

Happé, F., \& Loth, E. (2002). Theory of mind and tracking speakers' intentions. Mind and Language, 17, 24-36.

Harvard Mental Health Letter. (1999). What is Asperger's disorder? Harvard Mental Health Letter, 16, 8-12.

Healy, A., \& McNamara, D. (1996). Verbal learning and memory: Does the modal still work? Annual Review Psychology, 47, 143-172.

Heiman, G. (2002). Research methods in psychology ( $3^{\text {rd }}$ ed.). Boston: Houghton Mifflin.

Hitch, G., Towse, J., \& Hutton, U. (2001). What limits children's working memory span? Theoretical accounts and applications of scholastic development. Journal of Experimental Psychology, 130, 184-198.

Hitch, G., Woodin, M., \& Baker, S. (1989). Visual and phonological components of working memory in children. Memory and Cognition, 17, 175-185.

Holaday, M., Moak. J., \& Shipley, M. (2001). Rorschach protocols from children and adolescents with Asperger's disorder. Journal of Personality, 76, 482-495. 
Howlin, P., \& Asgharian, A. (1999). The diagnosis of autism and Asperger syndrome: Findings from a survey of 770 families. Developmental Medicine and Child Neurology, 41, 834-839.

Iwanaga, R., Kawasaki, C., \& Twuchida, R. (2000). Brief report: Comparison of sensorymotor and cognitive function between autism and Asperger syndrome and preschool children. Journal of Autism and Developmental Disorders, 30(2), n.p.

Jaccard, J., \& Becker, M. (1997). Statistics for the behavioral scientist ( $^{\text {rd }}$ ed.). Florence, KY: Brooks Cole.

Jolliffe, T., \& Baron-Cohen, S. (1999). The strange stories test: A replication with highfunctioning adults with autism or Asperger's syndrome. Journal of Autism and Developmental Disorders, 29, 395-406.

Jolliffe, T., \& Baron-Cohen, S. (2001). A test of central coherence theory: Can adults with high-functioning autism or Asperger's syndrome integrate objects in context? Visual Cognition, 8, 67-101.

Just, M., \& Carpenter, P. (1992). A capacity theory of comprehension: Individual differences in working memory, Psychological Review, 99, 122-149.

Kadesjo, B., Gillberg, C., \& Hagberg, B. (1999). Brief report: Autism and Asperger syndrome in seven-year-old children: A total population study. Journal of Autism and Developmental Disorders, 29, 327-331.

Kane, M., \& Engle, W. (2000). Working memory capacity, proactive interference and divided attention: Limits on long-term memory retrieval. Journal of Experimental Psychology: Learning Memory and Cognition, 26, 336-358.

Kane, M., Hambrick, D., Tuholski, S., Wilhelm, O., Payne, T., \& Engle, R. (2004). The generality of working memory capacity: A latent-variable approach to verbal and visual spatial memory span and reasoning. Journal of Experimental Psychology, General, 133, 189-217.

Kibby, M., Marks, W., Morgan, S., \& Long, C. (2004). Specific impairment in developmental reading disabilities: A working memory approach. Journal of Learning Disabilities, 37, 349-363.

Klin, A. (2000). Attributing social meaning to ambiguous visual stimuli in highfunctioning autism and Asperger's syndrome: The social attribution task. Journal of Child Psychology and Psychiatry, 41, 831-846. 
Koning, C., \& MaGill-Evans, J. (2001). Social and language skills in adolescent boys with Asperger syndrome. Autism: The International Journal of Research and Practice, 5, 23-41.

Lawson, J. (2003). Depth accessibility difficulties: An alternative conceptualization of autism spectrum conditions. Journal for the Theory of Social Behavior, 33, 189210.

Lieberman, M., \& Rosenthal, R. (2001). Why introverts can't always tell who likes them: Multitasking and nonverbal decoding. Journal of Personality and Social Psychology, 80, 294-310.

Little, L. (2003). Maternal perceptions of the importance of needs and resources for children with Asperger's syndrome and non-verbal learning disorders. Focus on Autism and Other Developmental Disorders, 4, 257-266.

Logie, R., \& Pearson, D. (1997). The inner eye and the inner scribe of visuo-spatial working memory: Evidence from developmental fractionation. European Journal of Cognitive Psychology, 9, 241-258.

Loisy, C., \& Roulin, J. (2003). Multiple short-term storage: A task to evaluate the coordination function of the central executive. International Journal of Psychology, 38, 1-10.

Marshall, M. (2002). Asperger's syndrome: Implications for nursing practice. Issues in Mental Health Nursing, 23, 605-615.

Marton, K., \& Schwartz, R. (2003). Working memory capacity and language processes in children with specific language impairment. Journal of Speech, Language and Hearing Research, 46, 1138-1153.

McConnell, J., \& Quinn, J. (2000). Interference in visual working memory. Quarterly Journal of Experimental Psychology, 1, 53-67.

Miles, C., Morgan, M., Milne, A., \& Morris, E. (1996). Developmental and individual differences in visual memory span. Current Psychology, 15, 53-67.

Miller, J., \& Ozonoff, J. (2000). The external validity of Asperger's disorder: Lack of evidence from the domain of neuro-psychology. Journal of Abnormal Psychology, 109, 227-238.

Milner, B. (1971). Inter-hemispheric differences in the localization of psychological processes in man. British Medical Bulletin, 27, 272-277. 
Miyake, A., Friedman, N., Rettinger, D., Shah, P., \& Hegarty, M. (2001). How are visuospatial working memory, executive functioning and spatial abilities related? A latent-variable analysis. Journal of Experimental Psychology, 130, 621-640.

Molloy, H., \& Vasil, L. (2002). The social construction of Asperger's syndrome: The pathologising of difference? Disability \& Society, 17, 659-669.

Morgan, B., Mayberry, M., \& Durkin, K. (2003). Weak central coherence, poor joint attention and low verbal ability: Independent deficits in early autism. Developmental Psychology, 39, 646-656.

Mueller, S., Seymour, T., Kieras, D., \& Meyer, D. (2003). Theoretical implications of articulatory duration, phonological similarity and phonological complexity in verbal working memory. Journal of Experimental Psychology, 29, 1353-1380.

Myles, B., \& Simpson, R. (2002). Asperger's syndrome: An overview of characteristics. Focus on Autism and Other Developmental Disabilities, 17, 132-137.

Neisser, U. (1967). Cognitive psychology. Upper Saddle River, NJ: Prentice Hall.

Nettle, D., \& Dunbar, R. (1997). Social markers and the evolution of reciprocal exchange. Current Anthropology, xx, 93-99.

Neuendorf, K. (2002). The content analysis guidebook. Thousand Oaks, CA: Sage.

Nunnally, J. C. (1978). Psychometric theory ( $2^{\text {nd }}$ ed.). New York: McGraw-Hill.

Ozonoff, S., Pennington, B., \& Rogers, S. (1991). Executive function deficits in high-functioning autistic individuals: Relationship to theory of mind. Journal of Child Psychology and Psychiatry and Allied Disciplines, 32, 1085-1105.

Park, D., Lautenschlager, G, Hedden, T., Davidson, N., Smith, P., \& Smith, A. (2002). Models of visuo-spatial and verbal memory across the adult life span. Psychology of Aging, 17, 299-320.

Parsons, S., \& Mitchell, P. (2002). The potential of virtual reality in social skills training for people with autistic spectrum disorders. Journal of Intellectual Disability Research, 46, 430-433.

Peterson, C., \& Siegal, M. (2002). Mind reading and moral awareness in popular and rejected preschoolers. Developmental Psychology, 20, 205-225.

Pickering, S. (2001a). The development of visual spatial working memory. Memory, 9, 423-432. 
Pickering, S. (2001b). Viewpoint: Cognitive approaches to the fractionation of visuospatial working memory. Cortex, 37, 457-473.

Pickering, S., \& Gathercole, S. (2001). Working memory test battery for children $(W M T B-C)$ manual. London, UK: Psychological Corporation.

Pickering, S., Gathercole, S., Hall, M., \& Lloyd, S. (2001). Development of memory for pattern and path: Further evidence for the fractionation of visuo-spatial memory. Quarterly Journal of Experimental Psychology, 54A, 397-420.

Plaisted, K., Swettenham, J., \& Ress, L. (1999). Children with autism show local precedence in a divided attention task and global precedence in a selective attention task. Journal of Psychology and Psychiatry, 40, 733-742.

Pope, K., \& Vasquez, M. (1998). Ethics in psychotherapy and counseling: A practical guide ( $2^{\text {nd }}$ ed.). San Francisco: Jossey-Bass.

Reuter-Lorenz, P., Jonides. J., Smith, E., Hartley, A., Miller, A., Marshuetz, C., et al. (2000). Age differences in the frontal lateralization of verbal and spatial working memory revealed by PET. Journal of Cognitive Neuroscience, 12, 174-187.

Rinehart, N., Bradshaw, J., Moss, Brereton, A., \& Tonge, B. (2000). Atypical interference of local detail on global processing in high functioning. Autism and Asperger's Disorder, 41, 769-778.

Rinehart, N., Bradshaw, J., Brereton, A., \& Tonge, B. (2002a). A clinical and neurobehavioral review of high-functioning autism and Asperger's disorder. High-Functioning Autism and Asperger's Disorder, 32, 762-770.

Rinehart, N., Bradshaw, J., Brereton, A., \& Tonge, B. (2002b). Laterization in individuals with high-functioning autism and Asperger's disorder: A frontostriatal model. Journal of Autism and Developmental Disorders, 32, 321-331.

Ropar, D., \& Mitchell, P. (2001). Do individuals with autism and Asperger's syndrome utilize poor knowledge when pairing stimuli? Developmental Science, 4, 433-441.

Rourke, B. (1989). Nonverbal learning disabilities: The syndrome and the model. New York: Guildford.

Rourke, B. (2000). Neuropsychological and psycho-social sub-typing: A review of investigations within the University of Windsor laboratory. Canadian Psychology, $41,34-50$. 
Rourke, B., Ahmad, S., Collins, D., Hayman-Abello, B., Hayman-Abello, S., \& Warriner, E. (2002). Child clinical/pediatric neuro-psychology: Some recent advances. Child Clinical Neuropsychology, 53, 309-339.

Safran, S., Safran, J., \& Ellis, K. (2003). Intervention ABC's for children with Asperger syndrome. Topics in Language Disorders, 23, 154-165.

Service, E. (1998). The effect of word length on immediate serial recall depends on phonological complexity, not articulatory duration. Quarterly Journal of Experimental Psychology, 51, 283-304.

Shulman, H. (1971). Similarity effects in short-term memory. Psychological Bulletin, 75, $399-415$.

Shah, P., \& Miyake, A. (1996). The separability of working memory resources for spatial thinking and language processing: An individual difference approach. Journal of Experimental Psychology: General, 125, 4-27.

Sicotte, C., \& Stemberger, R. (1999). Do children with PDD-NOS have a theory of mind? Journal of Autism and Developmental Disorders, 29, 225-233.

Smith, E., \& Jonides, J. (1999). Storage and executive processes in the frontal lobes. Neuroscience, 283, 1657-1661.

Stone, V., Baron-Cohen, S., \& Knight, R. (1998). Frontal lobe contributions to theory of mind. Journal of Cognitive Neuroscience, 10(5), 640-656.

Tager-Flusberg, H. (1999). A psychological approach to understanding the social and language impairments in autism. International Review of Psychiatry, 11, 325-334.

Tani, P., Lindberg, N., Joukamaa, M., Wendt, T., von Wendt, L., Appelberg, B., et al. (2004). Asperger syndrome, alexithymia and perception of sleep. Neuropsychobiology, 49, 64-70.

Towse, J., Hitch, G., \& Hutton, U. (1998). A re-evaluation of working memory capacity in children. Journal of Memory and Language, 39, 195-217.

Towse, J., Hitch, G., \& Hutton, U. (2000). On the interpretation of working memory span in adults. Memory and Cognition, 28, 341-348.

Trivers, R. (1971). The evolution of reciprocal altruism. Quarterly Review of Biology, 46, $35-57$. 
Turner, A., \& Engle, N. (1989). Is working memory capacity task dependent? Journal of Memory and Language, 28, 127-154.

Volkmar, F., Lord, C., Bailey, A., Schultz, R., \& Klin, A. (2004). Autism and pervasive developmental disorders. Journal of Child Psychology and Psychiatry, 45, 135170.

Wechsler, D. (2003). Wechsler Intelligence Scale for Children (4 ${ }^{\text {th }}$ ed.). San Antonio, TX: Psychological Corporation.

Welch, M., Pennington, B., \& Groisser, D. (1991). A normative-developmental study of executive function: A window on prefrontal function in children. Developmental Neuropsychology, 7, 131-149.

Wing, L., \& Potter, D. (2002). The epidemiology of autistic spectrum disorders: Is the prevalence rising? Mental Retardation and Developmental Disabilities Research Reviews, 8, 151-161.

World Health Organization. (1992). International classification of diseases $\left(10^{\text {th }}\right.$ ed.). Geneva: Author.

Yirmiya, N., Erel, O., Shaked, M., \& Solomonica-Levi, D. (1998). Meta-analysis comparing theory of mind abilities of individuals with autism, individuals with mental retardation and normally developing individuals. Psychological Bulletin, 124, 283-307. 


\title{
APPENDIX A
}

\section{Research Assistant Confidentiality Agreement}

\author{
Information Processing Skills of Individuals with Asperger's Disorder Related to \\ Working Memory \\ Walden University
}

The records of this study will be kept confidential and private. The research assistant will commit to maintaining the privacy of all participants involved in the study.

No identifying information will be disclosed regarding any of the participants nor will participation be confirmed or denied to parties other than the principal researcher or the research assistant.

All research materials will be delivered and kept in the possession of the principal researcher.

I agree to abide by the proceeding statements

Name of Research Assistant

Signature of Research Assistant and Date

Signature of Primary Researcher

Nancy Musarra (doctoral candidate at Walden University) 


\section{APPENDIX B}

\section{Solicitation of Participants}

Male students between the ages of 9 and 15 needed to participate in a research project that examines memory skills and communication. Interested individuals may contact Nancy Musarra at (216)xxx-xxxx or e-mail nmusarra@waldenu.edu, or contact your school counselor. 


\section{APPENDIX C}

\section{Parent Consent Form}

Information Processing Skills of Individuals with Asperger's Disorder related to Working

Memory

\section{Walden University}

You are invited to participate in a research study examining memory skills and communication of boys aged 9 to 15 . Your child was selected as a possible participant because of his age, gender (male) and social communication style. Please read this form and ask any questions you have before acting on this invitation to be in the study.

Nancy Musarra, doctoral candidate at Walden University, is conducting this study. Nancy Musarra is a Licensed Professional Clinical Counselor (LPCC) and appropriately trained to administer the assessments included in this study.

\section{BACKGROUND INFORMATION:}

The purpose of this study is to examine memory skills and communication styles of boys aged 9 through 15. This will demonstrate how memory skills contribute to the way in which boys between the ages of 9 through 15 communicate socially.

\section{PROCEDURES:}

If you agree to allow your child to be in this study, he will be asked to answer a variety of questions on a standardized memory assessment and a standardized assessment of general knowledge. The time required to complete each test is one to two hours.

\section{VOLUNTARY NATURE OF THE STUDY:}

Your child's participation in this study is strictly voluntary. Your decision whether or not to allow him to participate will not affect your current or future relations with your child's school, church or community. If you initially decide to participate, you are still free to withdraw later, at any time, without affecting those relationships. There will be no penalty for early withdrawal from the study.

\section{RISKS and BENEFITS of BEING in the STUDY}

This study does not present any significant risks.

However, in the event your child wants to discontinue participation in the study, he may ask to stop his participation at any time. He may refuse to answer any questions. 
Parents/Guardians will be notified immediately if data collection sessions are to be suspended. There will be no penalty for doing so.

The benefits of participation (child) in this study are to provide data for the parents and participant about strengths in memory skills and how memory skills relate to communication. The approximate cost of obtaining these assessments outside of this study by a licensed professional is about $\$ 800.00$ for test administration, scoring and interpretation.

VOLUNTARY:

This study is completely voluntary.

\section{CONFIDENTIALITY;}

The records of this study will be kept private. In any report of this study that might be published, the researcher will exclude any information that will make it possible to identify a participant. Research records will be kept in a locked file; only the researcher will have access to records.

\section{CONTACTS THE QUESTIONS}

The researcher conducting this study is Nancy Musarra, doctoral candidate at Walden University. The researcher's advisor is Dr. Rodney Ford. You may ask any questions you have now. If you have questions later, you may contact them with the following contact information:
Nancy Musarra LPCC
Rodney Ford Ph.D.
(216) xxx-xxxx
(248) xxx-xxxx
nmusarra@waldenu.edu
rford@waldenu.edu

The Research Participant Advocate at Walden University is Dr. Dale Good. You may contact him at 1-800-925-3368 x 1210 if you have questions about your participation in this study.

You will receive a copy of this form from the researcher and keep that copy for your records.

\section{STATEMENT OF CONSENT}

I have read the above information. I have asked questions and received answers. I consent to have my child participate in this study. 
Printed name of Parent or Guardian

Signature of Parent or Guardian $\quad$ DATE

Signature of Investigator

DATE 


\section{APPENDIX D}

\section{Child Consent Form}

Information Processing Skills of Individuals with Asperger's Disorder Related to

Working Memory

Walden University

You are invited to take part in a project. This project will look at memory skills and communication of boys between the ages of 9 through 15 .

To help me with this project you will be asked to complete two assessments with me that take about two hours each. Each assessment includes 10 mini tasks, games or questions that I will ask you to answer. We will schedule a meeting at the school to do the assessments. Your answers to the questions will be kept secret, in fact, you will be asked to make up a code name to put on your answer sheet.

How you answer the questions will not affect your relationships with your school, friends, community or parents.

If you begin to help with the project and you don't want to finish, it is okay to decide you don't want to finish. You won't be in trouble for not finishing the questions if you do not want to.

If you have any questions about this project, you can ask me or ask your parents to ask me. You can ask questions now or at any time.

I have read the above information. I have asked questions and received answers. I consent to take part in this study.

Name

Age Date 


\section{APPENDIX E}

\section{General Information Form}

Name of Student

Age Grade

Student's Date of Birth

School Contact

Parents/Guardians

Address

Phone numbers

Who lives with this student?

Does this student speak English yes no

Is this student Right or Left Handed?

Has this student ever been diagnosed with the following: Answer YES or NO

Substance Dependence

Schizophrenia

Hearing Impairment

The inability to articulate speech

Signature(s) of Person(s) Completing this form: 
CURRICULUM VITAE

\section{NANCY MUSARRA}

PROFESSIONAL EXPERIENCE

September 2002-Present

March 1993-1998

February 1992-1995

May 1987-Oct 1991

\section{EDUCATION}

September 2001-
The Center for Independence, LLC. Private Practice.

Psychological Assessment and Counseling Cuyahoga Heights HS

Safety and Self-Defense for Kids: (K-8).

Beyond Common Sense-Workshop series for professionals.

Psychological and Behavioral Consultants, Private Practice.

Individual and Family therapy; Children of divorce and marital relationships. Social skill teen groups, seminars and individual therapy.

Glenbeigh Health Sources, Cleveland, OH. Clinical coordinator of Dual Diagnosis Unit/ adults. Inpatient and Partial hospitalization program. Supervised graduate students from Case Western University, Baldwin Wallace and Ursuline College. Director of Expressive Arts Department: visual arts, drama and fitness.

Riverside Hospital, Columbus, $\mathrm{OH}$.

Art therapist: Adolescent Inpatients and Partial Hospitalization Adults.

Ph.D. Clinical Psychology, Walden University (pending June 2006)

Dissertation: Information-Processing Skills of Individuals with Asperger's Disorder Related to Working Memory.

Pre-doc Internship: Juvenile Diversion Program; Cuyahoga Heights School District: Psychological assessments and counseling for juvenile offenders. The Summit Academy: School for Autism, Asperger's and Attention Deficit Disorder students. Academic and Psychological testing. Program Development and Group therapy. 
JULY 1991

JUNE 1986

Master's of Science, University of Dayton, Social Agency Counseling.

Master's of Science, Hahnemann University, PA, Creative Arts Therapy.

Thesis Research: Art Therapy Techniques to Improve the Body Image of Children with SpinaBifida.

JUNE 1984

Bachelor of Science, Bowling Green State University, $\mathrm{OH}$.

Art therapy and Art Education (Certification K-12). J.K. Balogh Scholarship in Sculpture Arts.

\section{CREDENTIALS}

Sport Performance Consultant (AAASP): pending June, 2007.

American Taekwondo Association, Instructor in Training, Red Collar Licensed Professional Clinical Counselor Licensed Social Worker Registered Art Therapist Registered Recreational Therapist Aerobic Instructor Personal Trainer 